Research Article: New Research / Sensory and Motor Systems

\title{
Brain-wide synaptic inputs to aromatase- expressing neurons in the medial amygdala suggest complex circuitry for modulating social behavior
}

https://doi.org/10.1523/ENEURO.0329-21.2021

Cite as: eNeuro 2022; 10.1523/ENEURO.0329-21.2021

Received: 7 August 2021

Revised: 18 December 2021

Accepted: 26 December 2021

This Early Release article has been peer-reviewed and accepted, but has not been through the composition and copyediting processes. The final version may differ slightly in style or formatting and will contain links to any extended data.

Alerts: Sign up at www.eneuro.org/alerts to receive customized email alerts when the fully formatted version of this article is published.

Copyright @ 2022 Dwyer et al.

This is an open-access article distributed under the terms of the Creative Commons Attribution 4.0 International license, which permits unrestricted use, distribution and reproduction in any medium provided that the original work is properly attributed. 
1. Manuscript Title (50 word maximum): Brain-wide synaptic inputs to aromatase-expressing neurons in the medial amygdala suggest complex circuitry for modulating social behavior.

3

4

5

6

7

8

9 10

2. Abbreviated Title (50 character maximum) : Synaptic inputs to medial amygdala aromatase neurons.

3. List all Author Names and Affiliations in order as they would appear in the published article Joseph Dwyer ${ }^{1}$, Diane A. Kelly ${ }^{1,2}$, Joseph Bergan ${ }^{1,2}$

${ }^{1}$ Neuroscience and Behavior Program, University of Massachusetts, Amherst, Amherst MA, 01003, USA

${ }^{2}$ Department of Psychological and Brain Sciences, University of Massachusetts, Amherst, Amherst MA, 01003, USA

\section{Author Contributions:}

JFD: Designed research, Performed research, Analyzed data, Wrote the paper.

DAK: Designed research, Performed research, Analyzed data, Wrote the paper.

JFB: Designed research, Performed research, Analyzed data, Wrote the paper.

\section{Correspondence should be addressed to (include email address)}

Joseph F. Bergan: jbergan@umass.edu

6. Number of Figures: 9 Figures / 8 Supplementary Figures

7. Number of Tables: 3 Tables

8. Number of Multimedia: -NA

9. Number of words for Abstract: 152

\section{Number of words for Significance: 111}

\section{Number of words for Introduction: 750}

\section{Number of words for Discussion: 1800}

\section{Acknowledgements}

We acknowledge Catherine Dulac for the kind gift of the aromatase-cre line and the members of the Bergan lab for comments on earlier versions of this manuscript.

\section{Conflict of Interest: 'Authors report no conflict of interest'} 15. Funding sources:

HHS | NIH | National Institute of Mental Health (NIMH) R01MH115094-01A1

UMASS | University of Massachusetts Amherst (UMass Amherst) gift from H. Britton Sanderford Jr.

Armstrong Foundation 
Brain-wide synaptic inputs to aromatase-expressing neurons in the medial amygdala suggest complex circuitry for modulating social behavior.

\section{Abstract:}

Here, we reveal an unbiased view of the brain regions that provide specific inputs to aromataseexpressing cells in the medial amygdala, neurons which play an outsized role in the production of sex specific social behaviors, using rabies tracing and light-sheet microscopy. While the downstream projections from these cells are known, the specific inputs to the aromatase-expressing cells in the medial amygdala remained unknown. We observed established connections to the medial amygdala (e.g., bed nucleus of the stria terminalis and accessory olfactory bulb) indicating that aromatase neurons are a major target cell-type for efferent input including from regions associated with parenting and aggression. We also identified novel and unexpected inputs from areas involved in metabolism, fear and anxiety, and memory and cognition. These results confirm the central role of the medial amygdala in sex specific social recognition and social behavior and point to an expanded role for its aromatase-expressing neurons in the integration of multiple sensory and homeostatic factors, which are likely used to modulate many other social behaviors.

\section{Significance Statement:}

Aromatase-expressing neurons in the medial amygdala play a significant role in producing critically important social behaviors including parenting, aggression, and reproduction. We used rabies tracing and light-sheet microscopy to reveal an unbiased view of many brain regions that provide direct synaptic input to aromatase neurons and observe both well-established and previously unknown inputs from areas involved in parenting and aggression, metabolism, fear and anxiety, and memory and cognition. These results confirm the central role of the medial amygdala in social recognition behavior and point to an expanded role for its aromatase-expressing neurons in the integration of multiple sensory and homeostatic factors, which can be used to modulate many other social behaviors.

\section{Introduction:}

Social behaviors are necessary for communication, survival, and reproduction in species throughout the animal kingdom (Darwin, 1871; Marler, et al., 1979; Ueda et al., 2020), and depend on the integration of external and internal sensory cues. Nearly all sensory systems have been linked to social behavior (Keller, et al., 2006a; 2006b; 2006c; Pomerantz, et al., 1983; Contreras and Agmo, 1993; Strasser and Dixon, 1986). For example, the diverse, and often beautiful, vocalizations of songbirds signal their species, individual identity, territorial ownership, and readiness to mate (Marler, 1961). In humans, visual cues produced in the face and hands provide information about another's identity and emotional state (Haxby, et al., 2002). For many species, chemosensory cues are particularly important to detect, identify, and respond to social partners (Bradley, et al., 2002; Dulac, et al., 1995; Gonzalez, et al., 2017; Keller, et al., 2006c, Schwanzel-Fukuda, et al., 1991). In mice, chemosensory information, transduced by the main olfactory epithelium and the vomeronasal organ, provides the primary sensory input to a conserved network of brain regions dedicated to executing social behaviors (Newmann, et al., 1999).

The core brain regions that make up the social behavior network (SBN) are well-established and conserved for all vertebrate species examined thus far (Goodson, 2005). However, identifying the specific neural circuits that generate social behaviors has been challenging because of both the heterogeneity of cell populations in these regions and the interwoven and reciprocal nature of both the behaviors

49 themselves and the underlying circuits. Thus, a current goal in neuroscience is to define specific 
contributions of well-defined populations of neurons to circuit function and, ultimately, to behaviors. Genetic and viral tools allow precise characterization of the connections made by individual populations of neurons and have been instrumental in defining cell-type-specific wiring diagrams that mediate specific behaviors (Watabe-Uchida, et al., 2012; Menegas, et al., 2015; Kohl, et al., 2018; Wickersham, et al., 2007).

The medial amygdala (MeA), a central node in the SBN, receives sensory input directly from the accessory olfactory bulb (AOB) and the main olfactory bulb (MOB). The MeA integrates chemosensory signals with modulatory input from other brain regions (Ferguson, et al., 2001; Yao, et al., 2017) and circulating hormones (Cooke, et al., 1999; Cooke, et al., 2005) to guide behavioral responses through its projections to efferent behavioral centers (Wu, et al., 2009). MeA neurons in mice display sex differences in sensory responses to social stimuli (Bergan, et al., 2014; Samuelsen \& Meredith, 2009; Kim et al., 2017) and robust anatomical sex differences (Cooke, et al., 1999; Wu, et al., 2009; Billing et al., 2020). Social behaviors that are tightly associated with the MeA, including parenting, mate choice, and aggression, also display clear sex differences (Chen, et al., 2020; Vochteloo, et al., 1987; Yao, et al., 2017). Each of these behaviors are critical for survival and reproduction. Their dependence on an animal's sex, age, and neuroendocrine status offers an opportunity to understand how the function of a common neural circuit can be modified to meet the specific behavioral requirements of an individual animal.

A large population of MeA neurons express the enzyme aromatase, which converts testosterone to estradiol, is critical for aggression in both sexes, and is known to shape the pattern of sensory responses in the MeA (Unger et al., 2015; Bergan et al., 2014). To fully understand the role of aromatase neurons in social behavior, however, we must first identify the inputs they receive and the circuits they form. Past experiments have addressed the electrophysiology of MeA neurons (Binns, et al., 2005; Bergan et al., 2014; Yao et al., 2017; Meredith et al., 2004; Li, et al., 2018) as well as the efferent targets of the MeA aromatase population (Wu et al., 2009). Recently, a sex-specific connection from AOB mitral and tufted neurons to aromatase neurons of the MeA was identified using rabies-based viral tracing from aromatase neurons (Billing, et al., 2020). However, the MeA integrates information from a large network of brain regions. Here we use rabies-based circuit mapping in conjunction with whole-brain cleared tissue imaging to exhaustively characterize the sources of synaptic input to aromatase neurons in the $\mathrm{MeA}$, and to identify the information channels that shape aromatase-dependent social behaviors.

\section{Materials and Methods:}

Animal Use: All experiments were performed in strict compliance with the National Institute of Health. All animals were handled according to a protocol approved by the UMass Amherst Institutional Animal Care and Use Committee (IACUC; protocol \#2018-0014 and \#2017-0060).

Fifteen adult mice (2 to 8 months old, both male $(n=9)$ and female $(n=6)$ ) from an existing transgenic mouse line (Cyp19a1-Cre: Jax Labs) were housed in a temperature $\left(22^{\circ} \mathrm{C}\right)$ and light $(12 \mathrm{hr}$ : $12 \mathrm{hr}$ light: dark) controlled facility, with ad libitum access to food and water. The Cyp19a1-Cre transgenic line was generated by BAC recombination (Yao et al., 2017); its expression pattern faithfully recapitulates endogenous aromatase expression (Yao et al., 2017) and displays no known behavioral deficits in either heterozygous or homozygous animals.

Viral injections: We used a retrograde tracing system based on the modified rabies virus (EnvA-SAD $\Delta G-$ EGFP; Wickersham, et al., 2007) that uses two consecutive stereotaxic injections to visualize $\rightarrow \mathrm{MeA}^{\text {arom+ }}$. 
In the first injection, $500 \mathrm{nl}$ of AAV8-FLEX-TVA and AAV8-FLEX-RG-mCherry (mixed 1:1; Watabe-Uchida et al. 2012) were injected into either the left or right MeA (Bregma -1.9, Lateral 1.9, Depth 4.5 to 5.5). After 14 days, $500 \mathrm{nl}$ of SAD $\triangle$ G-EGFP(EnvA) virus (Viral Vector Core, Salk Institute, La Jolla, CA) was injected into the MeA at the same stereotaxic coordinates (Figure 2B; Wickersham et al. 2007). All stereotaxic injections used a cold capillary (20-micron diameter tip) coupled to a Narishige hydraulic manipulator (Wu et al., 2014). All AAV viruses were produced by the UNC Vector Core Facility (Chapel Hill, NC, United States).

The rabies virus requires the cell-surface expression of TVA to enter a cell and is therefore limited to infecting Cre-expressing neurons (Figure 2A). Once inside a neuron, the modified rabies virus requires co-expression of the envelope glycoprotein $(R G)$ to be packaged for retrograde trans-synaptic spread. The efficiency of viral infection was determined by injecting the same viruses into double-transgenic line that labels all Cre-expressing neurons with the R26-Isl-tdTomato reporter (Figure 2C; Madisen et al., 2010). This control experiment allowed us to estimate the infection rate (percent of the target population labeled by rabies; Figure 2D).

Tissue Processing: Ten days after the final injection, animals were deeply anesthetized with isoflurane and perfused with $50 \mathrm{ml}$ cold PBS followed by $25 \mathrm{ml}$ cold PFA ( $4 \%$ in PBS). The brain was extracted and post-fixed in $25 \mathrm{ml}$ hydrogel ( $4 \%$ acrylamide, $4 \%$ PFA, $0.05 \%$ bis acrylamide, and $0.25 \%$ VA-044 initiator suspended in $0.01 \mathrm{M}$ PBS; Isogai, et al., 2017) $4{ }^{\circ} \mathrm{C}$ overnight.

After 12 - 24 hours incubation in hydrogel, oxygen was flushed from the hydrogel by bubbling the liquid hydrogel solution with nitrogen. The tissue container was resealed and transferred to a $37^{\circ} \mathrm{C}$ water bath until polymerization was complete (at least two hours). Excess hydrogel was removed from the brain manually and the tissue sample was incubated to SDS-clearing solution $(10 \mathrm{mM}$ sodium dodecyl sulfate in $0.1 \mathrm{M}$ borate buffer, $\mathrm{pH}$ 8.5) for 2 days at $37^{\circ} \mathrm{C}$ prior to MHD-accelerated clearing (Dwyer, et al, 2019).

Active clearing was performed in a 5-gallon container containing SDS-clearing solution using a custom magnetohydrodynamic (MHD) clearing device (Dwyer et al., 2019) that rapidly removed unbound lipids from the tissue sample at $37^{\circ} \mathrm{C}$. Brains were cleared until bright white and translucent, which typically took 24-48 hours. Brains were then transferred to 0.01M PBS for $24 \mathrm{hr}$. Brains were transferred from PBS to an OptiView imaging solution (Isogai et al. 2017) with refractive index 1.45 and incubated at $37^{\circ} \mathrm{C}$ for 2 days before imaging.

Image acquisition and processing: Images were acquired with the Zeiss Z.1 Lightsheet microscope (Carl Zeiss, Jena, Germany). Rabies-labeled eGFP-expressing neurons were excited with a $488 \mathrm{~nm}$ laser. A $561 \mathrm{~nm}$ and/or $647 \mathrm{~nm}$ laser was used to produce an autofluorescence image for subsequent background subtraction and isolation of the GFP signal. Images were collected with a 5-times magnification objective lens with PCO-Edge scMOS cameras (PCO, Kelheim, Germany). The entire brain was imaged in the horizontal orientation from both the dorsal and ventral surfaces. This produced a series of slightly overlapping 3D image stacks for each brain. 3D Image stacks were saved at 1-5 $\mu \mathrm{m}$ resolution and reconstructed to form a 3D image of the entire brain using custom MATLAB scripts (Mathworks, Natick, $\mathrm{MA})$.

Cell-counting: Rabies-labeled cells were identified using a human-trained computer vision algorithm (llastik, Heidelberg University). Briefly, a training subset of images were annotated as 'not tissue', 'unlabeled tissue', and 'rabies labeled neuron' by a trained user to prepare a machine learning kernel to automatically identify rabies labeled neurons. The resulting kernel was refined over several iterations until it accurately identified rabies labeled cells. Ilastik then automatically classified each pixel in from each 
image based on its probability of being a member of each category. The resulting probability map for 'rabies labeled neuron' were segmented with 'regionprops' in MATLAB and the centroids of each identified neuron were identified. Automatically identified cells (ilastik, Heidelberg University) were reviewed and confirmed by a human observer for quality control.

Each identified 'input cell' was assigned to a brain region based on its anatomical location. Thus, each imaged brain was transformed into a list containing the numbers and positions of identified neurons in each brain region and for each experimental animal. The identified populations were interpreted as the afferent synaptic input to the target population. Our past successes with this strategy likely result from three factors: 1) Thousands of input neurons are typically labeled in each brain providing broad coverage of social circuits. 2) The genetic specificity, 'starter neurons', of our approach reduces variability introduced by cell-type heterogeneity and by fibers of passage at the injection site; 3) The automated analysis pipeline allows user to annotate a sample dataset, which is then used to efficiently and accurately segment all subsequent datasets into 'input cells', 'starter cells', and 'unlabeled tissue' in an unbiased manner (Menegas et al., 2015). Because of the intensely bright fluorescence of EGFP driven by the EnvA-SAD $\triangle$ G-EGFP virus, this algorithm can identify rabies labeled neurons with more than $99 \%$ accuracy and with very few false positives.

Brain Alignment: Each reconstructed 3D brain was aligned to the common framework Allen Brain Atlas (ccfv3; Wang, et al., 2020) using elastix (Klein, et al., 2010; Shamonin, et al., 2014). Custom MATLAB scripts (Mathworks, Natick, MA) were used to integrate user input and computer processing and produce the alignment. Transformix (Klein, et al., 2010; Shamonin, et al., 2014) was then used to migrate the identified cells in each brain to the position of the accurate brain region in the standard Allen Brain Atlas framework. This allowed us to identify the number of eGFP-expressing cells in each region of the brain in each animal.

Statistical Analysis: Statistical analyses were performed using MATLAB . Means are reported with standard error of the mean. Regressions were performed in MATLAB using the "fitlm" and "predict" functions. Regions identified as providing input to MeA arom+ neurons were determined using an single sample t-test on the observed cell counts in each region with Bonferroni correction for multiple comparisons when appropriate. A second comparison was made using a single sample t-test to determine if the input from a given region exceeded the expected input based on chance using the region volume and an even distribution of cells as the chance rates (see Table 3). Sex differences in synaptic input were first characterized using a repeated measures ANOVA with interactions conducted in MATLAB. Post-hoc analyses for sex differences were conducted using an exact Permutation test comparing the cell counts observed in males versus females for each region (Efron and Tibshirani, 1986). D' was calculated as a sensitivity index for distributions with different SEM by normalizing the difference in cell count means by the standard deviation of the overall distribution. All scripts required to replicate these analyses have been provided as supplementary materials.

\section{Results:}

We used a previously described aromatase-cre transgenic mouse line (Yao et al., 2017) crossed with the Al9 tdTomato reporter line (Madison, et al., 2010) to identify aromatase-expressing neurons throughout the brain (Figure 1). Consistent with previous reports, aromatase was expressed in a subset of cells largely restricted to the social behavior network with the largest population located in the posterodorsal MeA (MeApd; Figure 1C; Unger, et al., 2015; Yao, et al., 2017; Morris, et al.., 2008; Newman, et al., 
1999). We cleared the intact mouse brain with a modified version of CLARITY (Dwyer et al 2019, Chung et al., 2013), and imaged the whole brain using lightsheet microscopy (Isogai et al., 2017; Menegas et al., 2015). The full brain was digitally reconstructed and aligned to the Allen reference brain to allow automated categorization by region. This approach allowed us to identify labeled neurons throughout the brain and to maintain the macroscopic organization of these cells (Figure 1C).

\section{Population data: broad-scale brain regions}

Next, we identified the inputome for aromatase-expressing neurons located in the MeApd. The MeApd of adult male and female aromatase-cre mice was sterotaxically injected with double-floxed inverse orientation adeno-associated viruses that selectively express the EnvA receptor (TVA) and rabies glycoprotein $(R G)$ in aromatase-expressing neurons following cre-based recombination (Figure 2A; see methods). Two weeks later, an avian G-deleted rabies virus was injected in the same location (Rabies$\triangle$ G-eGFP). Endogenous tdtomato and eGFP-expressing 'starter neurons' were visible in the MeApd (Figure 2C). As above, rabies-infected brains were cleared, imaged with lightsheet microscopy, and aligned to the Allen reference atlas. GFP-positive neurons were automatically detected using a machine vision algorithm and manually curated to ensure accuracy (see methods; Menegas et al., 2015).

The number of neurons retrogradely labeled by rabies, which synapse directly on aromatase-expressing neurons in the MeApd $\left(\rightarrow \mathrm{MeA}^{\text {arom+ }}\right)$, were counted in all brain regions. The number of neurons labeled outside the MeA scaled linearly with the size of the initially infected 'starter neuron' population in the MeApd (Figure 2D; $B=2.6,[1.06,4.1] 95 \% \mathrm{Cl}, \mathrm{R}^{2}=0.45 ; \mathrm{F}=12.9, \mathrm{p}=0.002$ ). Injections were performed unilaterally in either the right or left hemisphere and the vast majority of $\rightarrow \mathrm{MeA}^{\text {arom+ }}$ neurons were ipsilateral, indicating that the circuit that provides input to aromatase-expressing neurons in the MeA is largely contained within a single hemisphere (Figure 2E; Table 1; Percentage of Ipsi cells $=98.9 \pm 0.24$ SEM; t-test: $p=0.0002$ ). Consistent with previous descriptions of inputs to the MeA (Scalia, et al., 1975) almost the entire identified inputome was restricted to subcortical regions (99.6\%; Figure $2 \mathrm{~F})$. Even when looking at finer regions that span the brain (e.g., hippocampal formation), we find nearly all cells confined to the most ventral areas (Figure 7A). Taken together, we found that each 'starter neuron' is associated with more than 100 presynaptic neurons on average, and the overwhelming majority of these neurons were both ipsilateral and subcortical.

\section{Synaptic input: coarse regions}

Aligning our whole brain datasets to the Allen reference atlas allowed us to identify the location of each neuron with high specificity. An individual dataset typically had neurons distributed across many subcortical regions. An example of neurons identified in a single animal is shown in Figure 3. We divided the Allen atlas into 10 mutually exclusive brain regions spanning the full volume of the reference brain and used these regions to coarsely classify the inputome for aromatase-expressing MeApd neurons (Figure 4A,B; see methods). Only statistically significant sources of synaptic input are reported, unless otherwise noted. The majority of $\rightarrow \mathrm{MeA}^{\text {arom+ }}$ were located in the cerebral nuclei $(59.7 \pm 1.4 ; \mathrm{t}(17)=10.8$, $\mathrm{p}<0.0001)$, with additional significant populations of $\rightarrow \mathrm{MeA}^{\text {arom+ }}$ neurons in the hypothalamus $(11 \pm 1.1$; $\mathrm{t}(17)=2.5, \mathrm{p}=0.01)$, hippocampus $(10 \pm 0.8 ; \mathrm{t}(17)=3.26, \mathrm{p}=0.0023)$, cortical subplate $(8.9 \pm 0.4 ; \mathrm{t}(17)=5.7$, $\mathrm{p}<0.0001)$ and olfactory regions $(7.2 \pm 0.35 ; \mathrm{t}(17)=5.2, \mathrm{p}<0.0001$; Figure $4 \mathrm{~B}, \mathrm{C})$. A small but consistent population of $\rightarrow \mathrm{MeA}^{\text {arom+ }}$ neurons were identified in the thalamus $(1.9 \pm 0.2 ; t(17)=2.05, p=0.03)$ and midbrain $(0.9 \pm 0.07 ; t(17)=3.4, p=0.0016)$. Very few neurons were identified in the isocortex $(0.36 \pm 0.03$; $t(17)=3.3, p=0.002)$, even though the isocortex and cerebellum represent the two largest regions of the brain by volume. Inputs from the hindbrain $(0.02 \pm 0.003 ; \mathrm{t}(17)=1.5, \mathrm{p}=0.076)$ and cerebellum $(0.009 \pm$ $0.002 ; t(17)=1, p=0.17)$ did not reach statistical significance. This indicates that the densities of neurons in 
the isocortex, cerebellum, midbrain, and hindbrain are very low while the densities of labeled neurons was highest in the cerebral nuclei, cortical subplate, and hypothalamus (Figure 4A; Figure 4-1). Because the midbrain, isocortex, hindbrain, and cerebellum each contained less than a single percent of the $\rightarrow \mathrm{MeA}^{\mathrm{arom}}$ they were excluded from the main dataset. We have included a detailed summary of all inputs from these minor regions in the supplementary materials (see Figures 4-2,4-3,4-4; Table 2). Comparison of the cell counts to cell density is included (Figures 4-1, 5-1, 6-1, 7-1,8-1).

\section{Synaptic input: finer-scale regions}

Closer investigation of five primary sources of input to the aromatase-expressing neurons in the MeApd revealed a consistent presynaptic circuit. $\rightarrow \mathrm{MeA}^{\text {arom+ }}$ neurons in the cerebral nuclei were initially divided into the striatum $(48.1 \pm 1.2 ; \mathrm{t}(17)=10, \mathrm{p}<0.0001)$ and pallidum $(11.6 \pm 0.6 ; \mathrm{t}(17)=4.7, \mathrm{p}<0.0001$; Figure $5 \mathrm{~A})$. Within the striatum, the MeA, including the 'starter neuron' population accounts for $20 \pm 1.3 \%$ of the total $\rightarrow \mathrm{MeA}^{\text {arom+ }}$ neurons in the brain (Figure $5 \mathrm{~B} ; \mathrm{t}(17)=3.8, \mathrm{p}=0.0007$ ). Regions that border the MeA, including CEA and CP, accounted for an additional $(11 \pm 0.6 ; \mathrm{t}(17)=4.7, \mathrm{p}<0.0001)$ and $(6.6 \pm 0.6$; $t(17)=2.6, p=0.01$ ) percentage of the overall inputome respectively (Figure $5 B$ ). Smaller populations of $\rightarrow \mathrm{MeA}^{\text {arom+ }}$ were identified in the IA $(0.43 \pm 0.04 ; \mathrm{t}(17)=2.7, \mathrm{p}=0.0078)$, nucleus accumbens $(0.15 \pm 0.014$; $t(17)=2.6, p=0.009)$, fundus of the striatum $(0.11 \pm 0.011 ; t(17)=2.5, p=0.01)$, lateral septal nucleus $(0.15 \pm$ $0.01 ; \mathrm{t}(17)=3, \mathrm{p}=0.0037)$, and septofimbrial nucleus $(0.05 \pm 0.006 ; \mathrm{t}(17)=2.0, \mathrm{p}=0.034)$ while the populations of $\rightarrow \mathrm{MeA}^{\text {arom+ }}$ in the rest of the striatal regions were not significant (Figure 5D). The largest pallidum projections include the globus pallidus, both external $(3.25 \pm 0.3 ; \mathrm{t}(17)=2.6, \mathrm{p}=0.009)$ and internal $(1.5 \pm 0.12 ; \mathrm{t}(17)=3.0, \mathrm{p}=0.004)$ segments; the substantia innominata $(2.5 \pm 0.2 ; \mathrm{t}(17)=3.2, \mathrm{p}=0.0025)$, the bed nuclei of the stria terminalis $(0.61 \pm 0.04 ; \mathrm{t}(17)=3.5, \mathrm{p}=0.001)$, and the diagonal band nucleus $(0.5 \pm$ $0.03 ; t(17)=3.6, p=0.001$; Figure $5 C$ ).

The hypothalamus provided the second largest source of $\rightarrow \mathrm{MeA}^{\text {arom }+}$ neurons with most inputs located in the lateral $(4.5 \pm 0.4 ; \mathrm{t}(17)=2.6, \mathrm{p}=0.001)$ and medial $(3.1 \pm 0.4 ; \mathrm{t}(17)=1.9, \mathrm{p}=0.04)$ zones, with a smaller contribution from the periventricular region $(1.0 \pm 0.06 ; \mathrm{t}(17)=3.9, \mathrm{p}=0.0006)$ and the periventricular zone $(0.25 \pm 0.03 ; \mathrm{t}(17)=2.4, \mathrm{p}=0.01$; Figure $6 \mathrm{~A})$. The two hypothalamic regions providing the largest number of $\rightarrow \mathrm{MeA}^{\text {arom+ }}$ neurons to the inputome are the lateral hypothalamic area $(2.0 \pm 0.17 ; \mathrm{t}(17)=3.0, \mathrm{p}=0.004)$ and ventromedial hypothalamic nucleus $(1.2 \pm 0.16 ; \mathrm{t}(17)=1.9, \mathrm{p}=0.04$; Figure $6 \mathrm{~A}$; Table 3$)$.

Both the amygdala and hippocampus are critical for memory and contain large populations of $\rightarrow M e A^{\text {arom }+}$ neurons. Hippocampal $\rightarrow \mathrm{MeA}^{\text {arom+ }}$ neurons are primarily located in field $\mathrm{CA} 3(6.3 \pm 0.6 ; \mathrm{t}(17)=2.7$, $p=0.007)$, followed by field CA1 $(2.5 \pm 0.2 ; t(17)=3.3, p=0.002)$ and CA2 $(0.26 \pm 0.2 ; t(17)=2.8, p=0.006$; Figure 7A). We also observed $\rightarrow \mathrm{MeA}^{\text {arom+ }}$ cells in the hippocampal-amygdaloid transition area $(0.22 \pm$ $0.02 ; \mathrm{t}(17)=2.7, \mathrm{p}=0.00$ ). The hippocampus is a large structure that spans nearly the entire dorsal to ventral axis of the brain. The vast majority of $\rightarrow \mathrm{MeA}^{\text {arom+ }}$ neurons in the hippocampus were located in the posterior ventral hippocampus (Figure 7A).

The largest proportion of inputs from the cortical subplate originate in amygdalar nuclei (Figure 7B), including the posterior amygdalar nucleus $(3.8 \pm 0.2 ; \mathrm{t}(17)=4.0, \mathrm{p}=0.0005)$ and lateral amygdala $(0.1 \pm$ $0.01 ; \mathrm{t}(17)=2.3, \mathrm{p}=0.017)$. The anterior and posterior basomedial $(1.6 \pm 0.1 ; \mathrm{t}(17)=3.3, \mathrm{p}=0.002 ; 1.6 \pm$ $0.1 ; \mathrm{t}(17)=3.4, \mathrm{p}=0.002 \mathrm{]}$ and anterior and posterior basolateral $(0.6 \pm 0.06 ; \mathrm{t}(17)=2.6, \mathrm{p}=0.009 ; 0.3 \pm$ $0.024 ; \mathrm{t}(17)=2.8, \mathrm{p}=0.006$ ) nuclei of the amygdala also each showed $\rightarrow \mathrm{MeA}^{\text {arom+ }}$ cells.

Within the olfactory areas (Figure 8A), the largest proportion of inputs originate in the cortical amygdalar $(4.0 \pm 0.3 ; \mathrm{t}(17)=3.8, \mathrm{p}=0.0007)$ and piriform $(1.0 \pm 0.06 ; \mathrm{t}(17)=4.4, \mathrm{p}=0.0002)$ areas, as well as the accessory olfactory bulb $(1.0 \pm 0.1 ; t(17)=2.4, p=0.014)$. Smaller contributions originate in the piriform 
amygdalar area $(0.38 \pm 0.03 ; \mathrm{t}(17)=4.5, \mathrm{p}=0.00017)$, the postpiriform transition area $(0.34 \pm 0.03$; $t(17)=2.8, p=0.0067)$.

The largest proportion of input cells originating in the thalamus (Figure 8B) come from the recticular nucleus $(0.9 \pm 0.15 ; \mathrm{t}(17)=1.6, \mathrm{p}=0.07)$ though this population was variable between individuals and not statistically significant. Additional input came from the ventral group of the dorsal thalamus $(0.33 \pm 0.04$; $t(17)=2.1, p=0.025)$, with smaller contributions originating from the geniculate groups of the dorsal thalamus $(0.2 \pm 0.03 ; \mathrm{t}(17)=1.2, \mathrm{p}=0.1)$ ventral thalamus $(0.08 \pm 0.01 ; \mathrm{t}(17)=2.1, \mathrm{p}=0.023)$, midline group of the dorsal thalamus $(0.11 \pm 0.01 ; \mathrm{t}(17)=2.7, \mathrm{p}=0.0002)$, and lateral group of the dorsal thalamus $(0.04 \pm$ $0.005 ; t(17)=2.0, p=0.03)$.

\section{Sex Differences in distribution of observed input cells}

Because sex differences in the MeA, and specifically in MeA ${ }^{\text {aromt }}$ neurons, are well established (Cooke, et al., 2005; Yao et al., 2017; Billing et al., 2019) we investigated sex differences in synaptic input to $\mathrm{MeA}^{\mathrm{arom}+}$ neurons. A regression analysis of the cell counts for all male regions against cell counts for all female regions indicated that the major regions providing synaptic input to $\mathrm{Me}^{\mathrm{arom}+}$ neurons are largely conserved across sexes (Figure $9 ; R^{2}=0.60252 ; F=39.4127 ; p<0.00001$; linear regression). No significant difference was observed for sex when considering all regions (ANOVA: $F=0.04 p=0.83$ ); however, there was a significant interaction between region and $\operatorname{sex}(A N O V A: F=1.81, p=0.01$ ) indicating that a subset of brain regions displayed sex differences in projections to $\mathrm{MeA}^{\text {arom+ }}$ neurons. Post-hoc analyses revealed nine regions that provided more input in males and one region that provided more input in females (Figure 9). $M e A^{\text {arom+ }}$ neurons received more input from the $A O B$, basolateral amygdala (both anterior and ventral), CA1 region of the hippocampus, piriform areas, diagonal band nucleus, and lateral preoptic area in males and more input from the ventral premammillary nucleus in females ( $p<0.05$ : permutation test; Table 4). Additionally, the fraction labeled neurons in the MeA, local to the injection site), was higher in females $(29.2 \pm 10.7)$ than males though this trend did not reach significance $(13.0 \pm 3.5 ; p=0.08$ : permutation test; Figure 9-1).

\section{Discussion:}

Social behaviors require an animal to detect and respond to specific stimuli that signal the identity of a conspecific: rivals, potential mates, and offspring each demand different behavioral responses. As a central component of the social behavior network the MeA plays an important role in interpreting chemicals used to convey social information and producing appropriate behavioral responses (Newman, et al., 1999; Bakker, et al., 2002; Yao, et al., 2017). Here, we focus on a population of neurons in the MeA that express the enzyme aromatase (Naftolin et al., 1975), which has been causally linked to social behaviors (Unger et al., 2015; Lee et al., 2014; Ishii et al., 2017). In this study we identify the broad constellation of synaptic inputs that allow aromatase-expressing MeA neurons to integrate social information. We show that these neurons receive input from an unexpectedly wide range of regions, with strong inputs from regions linked to chemosensation, memory, metabolism, and the networks underlying social behaviors and decision-making. (Figure 10)

Two regions that are classically associated with the MeA are the AOB and BNST (Scalia, et al., 1975). The MeA is often illustrated as the third node in the vomeronasal pathway: vomeronasal sensory neurons project to the AOB that in turn projects to the MeA (Scalia et al., 1975). Similarly, the BNST and MeA are together considered the extended amygdala, a collective region that processes sensory and social information (Marino, et al., 2016). Using classical retrograde tracing it has been previously shown that 
most AOB neurons project to the MeA (De Olmos, et al., 1978; Bergan, et al., 2014, with aromatase neurons receiving input overwhelmingly from the anterior subdivision of the AOB (Billing, et al., 2019). Our tracing results demonstrate a clear input to aromatase-expressing MeA neurons from both the $A O B$ and the BNST. However, the collective inputs from the AOB and BNST account for less than 2 percent of

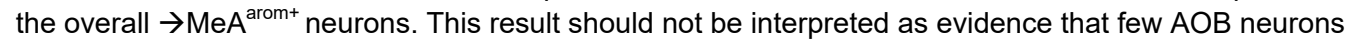
project to the MeA, but rather, it highlights the larger set of projections coming from areas other than the $\mathrm{AOB}$ and BNST. Aromatase-expressing neurons clearly have a wide-ranging role as integrators of information from inputs throughout the brain, illustrating the danger in thinking of the social behavior network as a simple feed-forward network. Moreover, our results indicate that many known postsynaptic targets of the MeA are also presynaptic inputs to aromatase expressing MeA neurons, suggesting that the MeA forms feedback loops with many regions in the social behavior network, even at the level of the population of aromatase-expressing neurons.

Nearly all $\rightarrow \mathrm{MeA}^{\text {arom+ }}$ neurons were located ipsilateral to the site of injection (Figure 1). This is consistent with previous findings (Canteras, et al., 1995; Bressler, et al., 1996; Bergan et al, 2014) and indicates that afferent sensory information from the left and right VNO is kept separate at least to the level of the MeA. The near complete ipsilateral bias seems surprising given the need to integrate both sides of the body in social behavior. One possibility is that chemical compounds activate each VNO similarly, reducing the need for integration between left and right sides. A second possibility is that, like the auditory system (Clemens, et al., 2015, Schnupp, et al., 2009) or visual system (Krapp, et al., 2001), internasal comparisons provide an additional line of sensory information (e.g. spatial location). In the latter case, our data suggests that internasal comparisons are likely done outside the MeA.

Despite representing more than $12.9 \%$ of the brain by volume, the cortex contained less than 0.5 percent of the total identified $\rightarrow \mathrm{MeA}^{\mathrm{arom}+}$ neurons. The only exception to this rule was the piriform cortex which processes olfactory information and provided clear input to aromatase neurons in the MeA, indicating an indirect route for arom+ neurons to integrate input from the main olfactory system with 'direct' vomeronasal input from the AOB. There is also a small input from the thalamus, which may represent an alternative pathway of directing cortical information to the arom+ MeA neurons. Indeed, integration between the main olfactory and vomeronasal systems is critical for adaptive social behaviors and these data indicate that inputs from both systems converge on $\mathrm{MeA}^{\text {arom+ }}$ with direct input from the $\mathrm{AOB}$ and multisynaptic input from the MOB. Consistent with the survival value of sex, parenting, and territorial defense, the brain regions that provide input to aromatase-expressing neurons are evolutionarily old and conserved (O'Connell, et al., 2011).

The vast majority of inputs to the aromatase-expressing MeA neurons were found in cerebral nuclei, hypothalamus, hippocampal formation, cortical subplate, and associated olfactory areas. The remaining regions collectively comprise less than $1.3 \%$ of total inputs. Thus, the inputome reported in this study suggests an integration of chemosensory input, emotional processing, memory, and sociosexual behavior regulation by $\mathrm{MeA}$ aromatase neurons. A combination of inputs from areas that provide information about the metabolic, reproductive, and attentional status of the animal implies a strong effect of the internal status of the animal on the activity of aromatase-expressing MeA cells that are likely integrated with input from regions involved in voluntary movement and the production of social behaviors. We will briefly discuss these specific inputs by their known functions in the following sections.

Areas involved in detecting chemosensory cues to drive specific sexual discrimination and sociosexual behaviors provide strong input to aromatase-expressing MeA neurons. Inputs from the ventromedial hypothalamus $(\mathrm{VMH})$, posterolateral and posteromedial cortical amygdala (PLCO, PMCO), anterior basomedial amygdala (aBMA), the nucleus of the lateral olfactory tract $(\mathrm{LT})$, and $A O B$ were identified 
(Nomoto and Lima 2015; Yang et al 2013; Maras and Petrulis 2008; Melis et al 2009; Hashikawa et al 2016; Moncho-Bogani et al 2005; Guo et al 2018). This result is consistent with previous data from MeA PLCO, AOB, and LT which are involved in the interpretation of chemosensory signals (Vaz, et al., 2017; Root, et al., 2014; Sosulki, et al., 2011).

Research into aggression, parenting, and sex consistently implicate the ventromedial hypothalamus (Kim, et al., 2019; Wang, et al., 2015) and medial preoptic area (Numan, 1988) as control regions for these behaviors. Recent work highlights the important role of galanin-expressing neurons of the MPOA for parental behavior (Wu, et al., 2014; Kohl, et al., 2018), and a primary source of sensory input for galaninexpressing neurons is the MeA.The MPOA is necessary to produce sex-specific mating and parenting behaviors (Wei, et al., 2018). The MPOA also plays a role in driving positive reinforcement for social interactions ( $\mathrm{Hu}$, et al., 2021). By identifying an arom+ MeA neuron-specific interaction between the MPOA and MeA, we can propose a loop between the MeA and MPOA with the potential to mediate positive and negative sex-specific patterns in parental and direct social interaction behaviors. Additional input to arom+ MeA neurons from the perifornical area (PeFA), BNST, and amygdalohippocampal area (AHi) further strengthen the role of the identified MeA arom+ circuitry for parental behavior (Kohl, et al., 2018; Autry, et al., 2019; Lebow, et al., 2016). neurons. Combined with previous data showing afferent inputs from the MeA (Pardo-Bellever, et al., 2012), these data demonstrate that aromatase-expressing MeA neurons have direct access to a parenting circuit.

Activation of $\mathrm{VMH}$ neurons causes immediate territorial aggression (Lin, et al., 2011): this is believed to be, at least in part, due to the role of ESR1-expressing neurons in the VMH (Lee, et al., 2014). A distinct aggressive behavior, pup-directed aggression, has been linked to the periforncal area (PeF; Autry, et al., 2019), amygdalohippocampal transition area (HATA), and ventromedial hypothalamus. Each of the aggression-linked regions contain neurons that project to aromatase neurons in the MeA, consistent with the observation that chemical silencing of aromatase-expressing neurons in the MeA directly reduces aggression in both male and female mice (Unger, et al., 2015).

Social interactions can be strongly influenced by fear and anxiety in both animals and people (Beery, et al., 2015; Sarnoff, et al., 1961). We found that several nodes of the amygdalar fear network send direct input to MeA aromatase neurons. The central amygdala (CeA), basomedial amygdalar nucleus (BMA), bed nucleus of the stria terminalis (BNST), and zona incerta (ZI) had some of the densest projections to the MeA in the brain; each is tightly linked to regulating anxiety and fear responses (Adhikari et al 2015; Amano et al 2010; Duvarci, et al., 2011; Chou, et al., 2018). Initially, this might suggest is that aromataseexpressing neurons are directly involved in the fear response to predators. We think this is not the case as the aromatase-expressing neurons receive sensory information from a class of V1R neurons not associated with predator responses (Billing, et al., 2020, Isogai, et al., 2011). Instead, we predict that these inputs are more likely sculpting behaviors directed towards conspecifics (mates, competitors, and pups) based on the proximity of predators (Apfelbach et al., 2005).

MeA aromatase neurons receive direct input from the hippocampus, known for its role in memory formation and consolidation (Scoville, et al., 1957). Hippocampal inputs to the MeA were not evenly distributed throughout the hippocampus, but rather were concentrated in the posterior ventral hippocampus including the HATA, CA1, CA2, and CA3. These are also the hippocampal regions anatomically closest to the MeA. Previous data indicate that region CA2 is particularly involved in memory and spatial processing but is also highly receptive to social state and plays a role in the deduction of social novelty (Dudek, et al., 2016). Similarly, the MeA has been linked to social memory (Ferguson et al., 2001) and MeA aromatase neurons have been directly implicated in social discrimination (Yao et al., 2017). We identified direct input from the ventral tegmental area, and together these sources of input may 
442

help elevate and reinforce responses to emotionally relevant stimuli (Zikopoulos, et al., 2012). We also identified inputs from areas involved in regulation of voluntary movement suggesting a potential for gating aromatase neuron-dependent behaviors.

A broad set of identified inputs to MeA aromatase neurons are associated with metabolic regulation, including the tuberal nucleus (TN), ventral premammillary nucleus (VPM), lateral hypothalamus (LH), and ventromedial hypothalamus (VMH). However, a hallmark of social behavior is that the pattern of social behaviors observed in young animals is different from those observed in adult animals. The transition from juvenile to adult behaviors depends on metabolic constraints including body weight and fat content (Castellano, et al., 2016). The observed input from brain regions that regulate metabolic state (Luo, et al., 2018; Donato, et al., 2010; Nisbett 1972; Berthoud and Munzberg 2011) further supports the role of the $\mathrm{MeA}$ as a node responsible for coordinating social behaviors in accord with internal state, reproductive readinesss, and environmental contexts (Padilla et al., 2016). This suggests that information about sexual receptivity and readiness are integrated with other relevant cues about conspecifics in the aromataseexpressing cells in the MeA.

In accord with the role of MeA, and aromatase neurons, in sexually dimorphic social behaviors, the MeA displays structural, molecular, and functional sex differences (Nishizuka and Arai, 1981; De Vries et al., 1984; Cooke et al., 1999; Morris et al., 2008; Bergan et al, 2014; Billing et al., 2019). In fact, the domestication process of creating isogenic mouse strains may have accentuated sex-differences in circuit function (Chalfin, et al., 2014; Bansal, et al., 2021). We found that the broad pattern of regional inputs to aromatase-expressing MeA neurons was conserved across males and females; however, a number of regions displayed a quantitative difference in the percentage of inputs observed in males as compared to females. Of the ten regions identified as having a quantitative sex-difference, nine had more labeled neurons in males. One possible explanation for this male bias is that the percentage of connections made by aromatase-expressing neurons inside the MeA is higher in females as compared to males. This hypothesis is partially supported by our data, which indicates more neurons labeled in the female MeA versus the male MeA; however, this trend did not reach significance. To account for variability in the levels of starter neurons infection, our analyses here rely on normalizing cell counts in each region by the overall number of identified neurons, and therefore, we cannot distinguish between a reduction in the number of local connections versus an enhancement of long-range connections.

The regions displaying sex differences provide new insights into the function of aromatase-expressing MeA circuits. The ventral premammillary nucleus was the only region identified with more neurons labeled in females and this region has recently been identified as a critical mediator of maternal aggression (Motta, et al., 2013). This is consistent with the role of aromatase-expressing MeA neurons in aggression in female mice (Unger et al., 2015). Regions with more neurons in males are linked to fear and anxiety (Yang, Y, et al., 2017), social and contextual memory (Okuyama, et al., 2016), mate identification and olfaction (Cádiz-Morretti, B., 2016; Wang, L, 2020), regulation of neuropeptide secretion (Brown, 2016), and male-specific sexual behavior (Schmidt, et al., 2000). Still, like the social behaviors mediated by $\mathrm{MeA}$ arom+ neurons in mice, the circuits that mediate these behaviors are sculpted differently in males and females, and these differences in circuit connectivity likely support sex differences in social behavior.

\section{Conclusions}

We comprehensively identified the inputome of aromatase-expressing MeA neurons, finding the sources of direct synaptic input to aromatase-expressing MeA neurons through rabies-based circuit mapping in the intact brains from transgenic animals expressing cre-recombinase under the control of the aromatase 
promoter. Presynaptic inputs were identified through a semiautomated algorithm, which provided an unbiased view of the circuit. Data from each individual is set in a standard reference frame that allows direct comparisons among brains, as well as to future datasets using a similar approach.

Our study confirms that known sources of synaptic input to MeA neurons (e.g., BNST and AOB) synapse specifically on arom+ neurons in the MeA. However, we also identified a broad set of subcortical inputs that have not been previously reported indicating that aromatase neurons in the MeA represent a critical node for integrating an array of internal states for the generation of social behaviors. The broad input to aromatase neurons reveals a framework that allows the aromatase-expressing MeA neurons to occupy a critical position in the social behavior network, with a role in nearly all known social behaviors ranging from parenting and reproduction to aggression and learning. While the overall pattern of inputs was similar in males and females, specific regions displayed sex differences in connectivity with aromataseexpressing MeA neurons further solidifying the role of these neurons as a control center for sex-specific social behaviors that are critical for survival.

\section{Data Availability:}

These data have been uploaded to ebrains online repository (Ref. 17110259) for future access and to allow reproducibility.

\section{References}

Abrahao, K., Chancey, J., Chan, C., Lovinger, D.M., (2017). Ethanol-Sensitive Pacemaker Neurons in the Mouse External Globus Pallidus. Neuropsychopharmacology 42, 1070-1081.

Adhikari, A., Lerner, T., Finkelstein, J., Pak, S., Jennings, J.H., Davidson, T.J., Ferenczi, E., Gunaydin, L.A., Mirzabekov, J.J., Ye, L., Kim, S.Y., Lei, A., Deisseroth, K., (2015). Basomedial amygdala mediates top-down control of anxiety and fear. Nature 527, 179-185

Amano, Duvarci, Popa, Paré, (2011). The Fear Circuit Revisited: Contributions of the Basal Amygdala Nuclei to Conditioned Fear. Journal of Neuroscience, 31(43):15481-9

Apfelbach R, Blanchard CD, Blanchard RJ, Hayes RA, McGregor IS. The effects of predator odors in mammalian prey species: a review of field and laboratory studies. Neurosci Biobehav Rev. 2005;29(8):1123-44. doi: 10.1016/j.neubiorev.2005.05.005. Epub 2005 Aug 8. PMID: 16085312.

Bakker, J., Honda, S., Harada, N., \& Balthazart, J. (2002). The aromatase knock-out mouse provides new evidence that estradiol is required during development in the female for the expression of sociosexual behaviors in adulthood. The Journal of neuroscience, 22(20), 9104-9112.

Baladron, Nambu, Hamker, (2017). The subthalamic nucleus-external globus pallidus loop biases exploratory decisions towards known alternatives: a neuro-computational study. European Journal of Neuroscience, 49(6):754-67

Balleine, Delgado, Hikosaka, (2007). The Role of the Dorsal Striatum in Reward and Decision-Making. Journal of Neuroscience, 27(31): 8161-65 
Bansal, R., Nagel, M., Stopkova, R., Sofer, Y., Kimchi, T., Stopka, P., Spehr, M., Ben-Shaul, Y., (2021). Do all mice smell the same? Chemosensory cues from inbred and wild mouse strains elicit stereotypic sensory representations in the accessory olfactory bulb. BMC Biol 19, 133

Beery and Kaufer, (2015). Stress, social behavior, and resilience: Insights from rodents. Neurobiology of Stress, 1:116-127

Bergan JF, Ben-Shaul Y, Dulac C. Sex-specific processing of social cues in the medial amygdala. Elife. 2014 Jun 3;3:e02743. doi: 10.7554/eLife.02743. PMID: 24894465; PMCID: PMC4038839.

Berthoud, Munzberg, (2011). The lateral hypothalamus as integrator of metabolic and environmental needs: From electrical self-stimulation to opto-genetics. Physiology and Behavior, 104(1):29-39

Billing, A, Correia, MH, Kelly, D, Li, G., and Bergan, (2019). Synaptic connections of aromatase circuits in the medial amygdala are sex-specific. Eneuro, eneuro.0489-19.2020

Binns, KE, Brennan, PA, (2005). Changes in electrophysiological activity in the accessory olfactory bulb and medial amygdala associated with mate recognition in mice. European Journal of Neuroscience, 21(9):2529-37.

Bressler and Baum, (1996). Sex comparison of neuronal fos immunoreactivity in the rat vomeronasal projection circuit after chemosensory stimulation. Neuroscience, 71(4):1063-72

Brown, C.H. (2016). Magnocellular Neurons and Posterior Pituitary Function. In Comprehensive Physiology, R. Terjung (Ed.).

Byrne, et al., (2008). Learning and Memory: A Comprehensive Reference. Elsevier, Academic Press. Cádiz-Moretti, B, Abellán-Álvaro, M., Pardo-Bellver, C., Martínez-García, Lanuza, E., (2016). Afferent and Efferent Connections of the Cortex-Amygdala Transition Zone in Mice. Frontiers in Neuroanatomy, $10: 125$

Canteras, Simerly, and Swanson, (1995). Organization of projections from the medial nucleus of the amygdala: A PHAL study in the rat. Journal of Comparative Neurology, 360(2):213-45

Castellano and Tena-Sempere (2016) Metabolic control of female puberty: potential therapeutic targets, Expert Opinion on Therapeutic Targets, 20:10, 1181-1193

Chalfin, L., Dayan, M., Levy, D. et al. Mapping ecologically relevant social behaviours by gene knockout in wild mice. Nat Commun 5, 4569 (2014).

Chen and Hong, (2018). Neural Circuit Mechanisms of Social Behavior. Neuron, 98(1):16-30

Chen, P. B., Hu, R. K., Wu, Y. E., Pan, L., Huang, S., Micevych, P. E., \& Hong, W. (2019). Sexually Dimorphic Control of Parenting Behavior by the Medial Amygdala. Cell, 176(5), 1206-1221.e18.

Chou, XI., Wang, X., Zhang, Zg., Shen, L., Zingg, B., Huang, J., Zhong, W., Mesik, L., Zhang, L.I., Tao, H.W., (2018). Inhibitory gain modulation of defense behaviors by zona incerta. Nat Commun 9, 1151 
Chung, K., Wallace, J., Kim, SY., Kalyanasundaram, S., Andalman, A.S., Davidson, T.J., Mirzabekov, J.J., Zalocusky, K.A., Mattis, J., Denisin, A.K., Pak, S., Bernstein, H., Ramakrishnan, C., Grosenick, L., Gradinaru, V., Deisseroth, K., (2013). Structural and molecular interrogation of intact biological systems. Nature 497, 332-337

Clemens, Girardin, Coen, Guan, Dickson, and Murthy, (2015). Connecting Neural Codes with Behavior in the Auditory System of Drosophila. Neuron, 87(6):1332-43

Contreras JL, Agmo A. Sensory control of the male rat's copulatory thrusting patterns. Behav. Neural Biol. 1993;60:234-240.

Cooke and Woolley, (2005). Sexually Dimorphic Synaptic Organization of the Medial Amygdala. Journal of Neuroscience. 25(46): 10759-67.

Cooke, B, Tabibnia, G, and Breedlove, M, (1999). A brain sexual dimorphism controlled by adult circulating androgens. PNAS, 96(13):7538-40.

De Olmos J, Hardy H, Heimer L (1978) The afferent connections of the main and accessory olfactory bulb formations in the rat. An experimental HRP study. J Comp Neurol 181:213-244

De Vries GJ, Buijs RM, Van Leeuwen FW (1984) Sex differences in vasopressin and other neurotransmitter systems in the brain. Prog Brain Res 61: 185-203.

Di Marino V., Etienne Y., Niddam M. (2016) The Concept of “Extended Amygdala”. In: The Amygdaloid Nuclear Complex. Springer, Cham. Donato jr., Cravo, Frazao, Gautron, Scott, Lachey, Castro, Margatho, Lee, S., Lee, C., Richardson, J. A., Friedman, J., Chua Jr, S., Coppari, R., Zigman, J.M., Elmquist, J.K.,

Elias, C.F., (2010). Leptin's effect on puberty in mice is relayed by the ventral premammillary nucleus and does not require signaling in Kiss1 neurons. The Journal of Clinical Investigation, 121(1):355-68

Donato jr., Elias, (2011). The ventral premammillary nucleus links metabolic cues and reproduction. Frontiers in endocrinology, 57(2):1-10

Dudek, S. M., Alexander, G. M., \& Farris, S. (2016). Rediscovering area CA2: unique properties and functions. Nature reviews. Neuroscience, 17(2), 89-102. Dulac and Axel (1995). A novel family of genes encoding putative pheromone receptors in mammals. Cell, 83:195-206.

Dwyer, J, Ramirez, MD, Katz, PS, Karlstrom, RO, Bergan, J, (2019). Accelerated clearing and molecular labeling of large tissue samples using magnetohydrodynamic force. bioRxiv 819292;

B. Efron. R. Tibshirani. (1986). Bootstrap Methods for Standard Errors, Confidence Intervals, and Other Measures of Statistical Accuracy. Statist. Sci. 1 (1) $54-75$

Fadel and Deutch, (2002). Anatomical substrates of orexin-dopamine interactions: lateral hypothalamic projections to the ventral tegmental area. Neuroscience, 111(2):379-387.

Ferguson, J, Aldag, J, Insel, T, Young, L, (2001). Oxytocin in the Medial Amygdala is Essential for Social Recognition in the Mouse. Journal of Neuroscience, 21(20):8278-85. 
Fernandez, LMJ, Vantomme, Osorio-Forero, Cardis, Beard, Lüthi, A., (2018). Thalamic reticular control of local sleep in mouse sensory cortex. eLife 2018;7:e39111

Ferretti, Maltese, Contarini, Nigro, Bonavia, Huang, Gigliucci, Morelli, Scheggia, Managó, Castellini, Lefevre, Cancedda, Chini, Grinevich, Papaleo, (2019). Oxytocin Signaling in the Central Amygdala Modulates Emotion Discrimination in Mice. Current Biology, 29(12):1938-53.e6

Field, Rosenstock, King, Greene, (1978). Behavioral role of the mammillary efferent system. Brain Research Bulletin, 3(5): 451-6

Gonzalez, F., Witzgall, P. \& Walker, W. (2017). Antennal transcriptomes of three tortricid moths reveal putative conserved chemosensory receptors for social and habitat olfactory cues. Sci Rep 7, 41829

Goodson J. L. (2005). The vertebrate social behavior network: evolutionary themes and variations. Hormones and behavior, 48(1), 11-22.

Guo, X., Guo, H., Zhao, L., Zhang, Y.H., Zhang, J.X., (2018). Two predominant MUPs, OBP3 and MUP13, are male pheromones in rats. Front Zool 15, 6. Haller, (2018). The role of central and medial amygdala in normal and abnormal aggression: A review of classical approaches..Neuroscience and Biobehavior Reviews, 85:34-43

Haller, (2018). The role of central and medial amygdala in normal and abnormal aggression: A review of classical approaches. Neuroscience and Biobehavior Reviews, 85:34-43

Haller, J, Tóth, Halasz, De Boer, (2006). Patterns of violent aggression-induced brain c-fos expression in male mice selected for aggressiveness. Physiology and Behavior 88(1-2):173-182

Hammen, G, Turaga, D, Holy, T, Meeks, J, (2014). Functional organization of glomerular maps in the mouse accessory olfactory bulb. Nature neuroscience 17, 953-61.

Han, Tellez, Rangel jr., Motta, Zhang, Perez, I.O., Canteras, N.S., Shammah-Lagnado, S.J., van den Pol, A.N., de Araujo, I.E., (2017). Integrated Control of Predatory Hunting by the Central Nucleus of the Amygdala. Cell 168(1-2): 311-324.e18

Harris, G., Wimmer, M. \& Aston-Jones, G. A role for lateral hypothalamic orexin neurons in reward seeking. Nature 437, 556-559 (2005). Hashikawa, K, Hashikawa, Y, Falkner, Lin, (2016). The neural circuits of mating and fighting in male mice. Current Opinion in Neurobiology, 38:27-37

Haxby, J, Hoffman, E, Gobbini, M, (2002). Human neural systems for face recognition and social communication. Biological Psychiatry, 51(1):59-67.

Horiai, M., Otsuka, A., Hidema, S., Hiraoka, Y., Hayashi, R., Miyazaki, S., Furuse, Mizukami, H., Teruyama, R., Tamura, M., Bito, H., Maejima, Y., Shimomura, K., Nishimori, K., (2020). Targeting oxytocin receptor (Oxtr)-expressing neurons in the lateral septum to restore social novelty in autism spectrum disorder mouse models. Sci Rep 10, 22173

Hu, R.K., Zuo, Y., Ly, T., Wang, J., Meera, P., Wu, Y.E., Hong, W., (2021). An amygdala-to-hypothalamus circuit for social reward. Nat Neurosci 
Hunt, A.J., Dasgupta, R., Rajamanickam, S., Jiang, Z., Beierlein, M., Chan, C.S., Justice, N.J., (2018). Paraventricular hypothalamic and amygdalar CRF neurons synapse in the external globus pallidus. Brain Struct Funct 223, 2685-2698

Ishii KK, Osakada T, Mori H, Miyasaka N, Yoshihara Y, Miyamichi K, Touhara K. A Labeled-Line Neural Circuit for Pheromone-Mediated Sexual Behaviors in Mice. Neuron. 2017 Jul 5;95(1):123-137.e8. doi: 10.1016/j.neuron.2017.05.038. Epub 2017 Jun 22. PMID: 28648498.

Isogai, Y., Richardson, D., Dulac, C., and Bergan, J.F. (2017) Optimized protocol for imaging cleared neural issues using light microscopy, in Synapse Development, Methods in Molecular Biology, Springer, 1538: 137-153.

Isogai, Y., Si, S., Pont-Lezica, L., Tan, T., Kapoor, V., Murthy, V. N., \& Dulac, C. (2011). Molecular organization of vomeronasal chemoreception. Nature, 478(7368), 241-245. Jasnow, Schulkin, Pfaff, (2006). Estrogen facilitates fear conditioning and increases corticotropin-releasing hormone mRNA expression in the central amygdala in female mice. Hormones and Behavior, 49(2):197-205

Ji, J., \& Maren, S. (2008). Differential roles for hippocampal areas CA1 and CA3 in the contextual encoding and retrieval of extinguished fear. Learning \& memory (Cold Spring Harbor, N.Y.), 15(4), 244251.

Keller M, Douhard Q, Baum MJ, Bakker J. Destruction of the main olfactory epithelium reduces female sexual behavior and olfactory investigation in female mice. Chem. Senses. 2006a;31:315-323.

Keller M, Douhard Q, Baum MJ, Bakker J. Sexual experience does not compensate for the disruptive effects of zinc sulfate--lesioning of the main olfactory epithelium on sexual behavior in male mice. Chem. Senses. 2006b;31:753-762.

Keller M, Pierman S, Douhard Q, Baum MJ, Bakker J. The vomeronasal organ is required for the expression of lordosis behaviour, but not sex discrimination in female mice. Eur $\mathrm{J}$ Neurosci. 2006c;23:521-530.

Kim, Im, (2018). The role of the dorsal striatum in choice impulsivity. Anals of the New York academy of sciences, 1451(1):92-111

Kim, Y, Yan, GR, Pradhan, K, Venkataraju, KU, Bota, García Del Molino, L.C., Fitzgerald, G., Ram, K., He, M., Levine, J.M., Mitra, P., Huang, Z., Wang, X.J., Osten, P., (2017). Brain-wide Maps Reveal Stereotyped Cell Type-based Cortical Architecture and Subcortical Sexual Dimorphism. Cell, 171(2)-45669.e22

King, (2006). The rise, fall, and resurrection of the ventromedial hypothalamus in the regulation of feeding behavior and body weight. Physiology and Behavior, 87(2):221-44

Klein, Staring, Murphy, Viergever, Pluim. elastix: a toolbox for intensity based medical image registration. IEEE Transactions on Medical Imaging, 29(1): 196 - 205

Kohl, J., \& Dulac, C. (2018). Neural control of parental behaviors. Current opinion in neurobiology, 49, 116-122. Kohl, J., Autry, A. E., \& Dulac, C. (2017). The neurobiology of parenting: A neural circuit 
perspective. BioEssays : news and reviews in molecular, cellular and developmental biology, 39(1), 1-11. doi:10.1002/bies.201600159

Krapp, Hengstenberg, and Egelhaaf, (2001). Binocular Contributions to Optic Flow Processing in the Fly Visual System. Journal of Neurophysiology, 85(2):724-34

Lanciego JL, Luquin N, Obeso JA. Functional neuroanatomy of the basal ganglia. Cold Spring Harb Perspect Med. 2012;2(12):a009621. Published 2012 Dec 1. doi:10.1101

Lee, H., Kim, D. W., Remedios, R., Anthony, T. E., Chang, A., Madisen, L., Zeng, H., \& Anderson, D. J. (2014). Scalable control of mounting and attack by Esr1+ neurons in the ventromedial hypothalamus. Nature, 509(7502), 627-632. Lee, JH., Latchoumane, CF.V., Park, J., Kim, J., Jeong, J., Lee, K.W., Shin, H.S., (2019). The rostroventral part of the thalamic reticular nucleus modulates fear extinction. Nat Commun 10, 4637

Li and Dulac, (2018). Neural coding of sex-specific social information in the mouse brain. Current Opinion in Neurobiology, 53:120-30

Lin, D., Boyle, M., Dollar, P., Lee, H., Lein, E.S., Perona, P., Anderson, D.J., (2011). Functional identification of an aggression locus in the mouse hypothalamus. Nature 470, 221-226

Luo, M, Fee, M, and Katz, L, (2003). Encoding Pheromonal Signals in the Accessory Olfactory Bulb of Behaving Mice. Science, 299(5610):1196-1201

Luo, SX, Huang, J, Li, Q, Mohammad, H, Lee, Chun-Yao, Krishna, K., Kok, A.M.Y., Tan, Y.L., Lim, J.Y., Li, H., Yeow, L.Y., Sun, J.J., He, M., Jean, J.G., Sajikumar, S., Han, W., Fu, Y., (2018). Regulation of feeding by somatostatin neurons in the tuberal nucleus. Science, 361(6397):76-81

M. A., and A. Chen. "Overshadowed by the amygdala: the bed nucleus of the stria terminalis emerges as key to psychiatric disorders." Molecular psychiatry 21.4 (2016): 450

Madisen L; Zwingman TA; Sunkin SM; Oh SW; Zariwala HA; Gu H; Ng LL; Palmiter RD; Hawrylycz MJ; Jones AR; Lein ES; Zeng H. 2010. A robust and high-throughput Cre reporting and characterization system for the whole mouse brain. Nat Neurosci 13(1):133-40

Malvaez, Wassum, (2018). Regulation of habit formation in the dorsal striatum. Current Opinon in Behavioral Sciences, 20:67-74

Maras, Petrulis, (2008). The posteromedial cortical amygdala regulates copulatory behavior, but not sexual odor preference, in the male Syrian hamster (Mesocricetus auratus). Neuroscience, 156(3):425-35

Marler, P (1961). The logical analysis of animal communication. J. Theoret. Bioi. $1: 295$ - 317

McCarthy MM. Molecular aspects of sexual differentiation of the rodent brain. Psychoneuroendocrinology. 1994;19(5-7):415-27. doi: 10.1016/0306-4530(94)90029-9. PMID: 7938343.

Menegas, W, Bergan, J, Ogawa, S, Isogai, Y, Venkataraju, K.U., Osten, P., Uchida, N., Watabe-Uchida, M., (2015). Dopamine neurons projecting to the posterior striatum form an anatomically distinct subclass. eLife 2015;4:e10032. 
Meredith and Westberry, (2004). Distinctive Responses in the Medial Amygdala to Same-Species and Different-Species Pheromones. Journal of Neuroscience, 24 (25) 5719-5725

Mesquita, Abreu, Rezende de Abreu, Arlindo de Souza, Rendeiro de Noronha, Silva, F.C., Campos, G.S.V., Chianca Jr, D.A., de Menezes, R.C., (2016). New insights on amygdala: Basomedial amygdala regulates the physiological response to social novelty. Neuroscience, 330:181-190

Moncho-Bogani, J, Martinez-Garci, Novejarque, Lanuza, (2005). Attraction to sexual pheromones and associated odorants in female mice involves activation of the reward system and basolateral amygdala. European Journal of Neuroscience, 21(8):2186-98 Morris JA, Jordan CL, Breedlove SM, (2008). Sexual dimorphism in neuronal number of the posterodorsal medial amygdala is independent of circulating androgens and regional volume in adult rats. $\mathrm{J}$ Comp Neurol. 506(5):851-9.

Morris, Jordan, King, Northcutt, Breedlove, (2008). Sexual dimorphism and steroid responsiveness of the posterodorsal medial amygdala in adult mice. Brain Research, 1190:115-121

Motta, S.C., Guimarães, C.C., Furigo, I.C., Sukikara, M.H., Baldo, M.V.C., Lonstein, J.S., Canteras, N.S., (2013). Ventral premammillary nucleus as a critical sensory relay to the maternal aggression network. PNAS, 110(35):14438-43

Newman SW. The medial extended amygdala in male reproductive behavior: A node in the mammalian social behavior network. Ann NY Acad Sci. 1999;877:242-257.

Nisbett, R. E. (1972). Hunger, obesity, and the ventromedial hypothalamus. Psychological Review, 79(6), 433-453. Nishizuka M, Arai Y. (1981). Sexual dimorphism in synaptic organization in the amygdala and its dependence on neonatal hormone environment. Brain Res.212(1):31-8.

Nomoto, Lima, (2015). Enhanced Male-Evoked Responses in the Ventromedial Hypothalamus of Sexually Receptive Female Mice. Current Biology, 25(5):589-94

Numan, (1988). Neural basis of maternal behavior in the rat. Psychoneuroendocrinology, 13(1-2):47-62

O'connell and Hofmann, (2011). Genes, hormones, and circuits: An integrative approach to study the evolution of social behavior. Frontiers in Neuroendocinology, 32(3):320-35

Okuyama, T., Kitamura, T., Roy, D. S., Itohara, S., \& Tonegawa, S. (2016). Ventral CA1 neurons store social memory. Science, 353(6307), 1536-1541.

Padilla SL, Quu J, Soden ME, Sanz E, Nestor CC, Barker FD, Quintana A, Zweifel LS, Rønnekleiv OK, Kelly MJ, Palmiter RD (2016). Agouti-related peptide neural circuits mediate adaptive behaviors in the starved state. Nat Neurosci. 19(5):734-741. doi: 10.1038/nn.4274.

Palmiter R. D. (2008). Dopamine signaling in the dorsal striatum is essential for motivated behaviors: lessons from dopamine-deficient mice. Annals of the New York Academy of Sciences, 1129, 35-46. 
Pardo-Bellver C, Cádiz-Moretti B, Novejarque A, Martínez-García F and Lanuza E (2012) Differential efferent projections of the anterior, posteroventral, and posterodorsal subdivisions of the medial amygdala in mice. Front. Neuroanat. 6:33. doi: 10.3389/fnana.2012.00033

Pinault, (2004). The thalamic reticular nucleus: structure, function and concept. Brain Research Reviews, $46(1): 1-31$

Pisa M. Motor functions of the striatum in the rat: critical role of the lateral region in tongue and forelimb reaching. Neuroscience. 1988 Feb;24(2):453-63. doi: 10.1016/0306-4522(88)90341-7. PMID: 3362348.

Pomerantz SM, Nunez AA, Bean NJ. Female behavior is affected by male ultrasonic vocalizations in house mice. Physiol Behav. 1983;31:91-96.

Qiu, MH, Chen, MC, Wu, J, Nelson, D, Lu, J, (2016). Deep brain stimulation in the globus pallidus externa promotes sleep. Neuroscience, 322(13):115-120

Root, C., Denny, C., Hen, R., Axel, R., (2014). The participation of cortical amygdala in innate, odourdriven behaviour. Nature 515, 269-273

Ross, Leon, Madara, JC, Schafer, Fergani, et al., (2018). PACAP neurons in the ventral premammillary nucleus regulate reproductive function in the female mouse. eLife 2018;7:e35960

Samuelson and Meredith (2009). The vomeronasal organ is required for the male mouse medial amygdala response to chemical-communication signals, as assessed by immediate early gene expression. Neuroscience, 164(4):1468-74

Sarnoff, I., \& Zimbardo, P. G. (1961). Anxiety, fear, and social isolation. The Journal of Abnormal and Social Psychology, 62(2), 356-363.

Scalia and Winans, (1975). The differential projections of the olfactory bulb and accessory olfactory bulb in mammals. The journal of comparative neurology, 161(1):31-55

Schmidt, M.H., Valatx, J.L., Sakai, K., Fort, P., Jouvet, M., (2000). Role of the Lateral Preoptic Area in Sleep-Related Erectile Mechanisms and Sleep Generation in the Rat. JNeurosci, 20(17):6640-7

Schnupp and Carr, (2009). On hearing with more than one ear: lessons from evolution. Nature neuroscience, 12(6):692-7

Schroll, Horn, Grüschel, Brücke, Lütjens, Schneider, Krauss, J.K., Kühn, A.A., Hamker, F.H., (2015). Differential contributions of the globus pallidus and ventral thalamus to stimulus-response learning in humans. Neurolmage, 122(15):233-45

Schwanzel-Fukuda, Marlene; Pfaff, Donald W. (1991). Migration of LHRH-immunoreactive neurons from the olfactory placode rationalizes olfacto-hormonal relationships. , 39(4), 565-572. doi:10.1016/09600760(91)90254-3

Scoville, W. B. \& Milner, B. Loss of recent memory after bilateral hippocampal lesions. J. Neurol. Neurosurg. Psychiatry 20, 11-21 (1957). 
Shamonin, DP, Bron, Lelieveldt, Smits, Klein and Staring. Fast Parallel Image Registration on CPU and GPU for Diagnostic Classification of Alzheimer's Disease. Frontiers in Neuroinformatics, 7(50):1-15

Sosulski, D., Bloom, M., Cutforth, T., Axel, R., Datta, S.R., (2011). Distinct representations of olfactory information in different cortical centres. Nature 472, 213-216

Stettler, D, Axel, R, (2009). Representations of Odor in the Piriform Cortex. Neuron, 63(6):854-64

Strasser S, Dixon AK. Effects of visual and acoustic deprivation on agonistic behaviour of the albino mouse (M. musculus L.) Physiol Behav. 1986;36:773-778.

Stuber, G., Wise, R. Lateral hypothalamic circuits for feeding and reward. Nat Neurosci 19, 198-205 (2016). Ueda, HR, Dodt, HU, Osten, P, Economo, MN, Chandrashekar, J, Keller, P.J., (2020). WholeBrain Profiling of Cells and Circuits in Mammals by Tissue Clearing and Light-Sheet Microscopy. Neuron, 106(3):369-87

Unger, E. K., Burke, K. J., Jr, Yang, C. F., Bender, K. J., Fuller, P. M., \& Shah, N. M. (2015). Medial amygdalar aromatase neurons regulate aggression in both sexes. Cell reports, 10(4), 453-462. Vann, (2010). Re-evaluating the role of the mammillary bodies in memory. Neurophyschologia, 48(8):2316-27

Vaz, R.P., Cardoso, A., Sá, S.I., Pereira, P.A., Madeira, M.D., (2017). The integrity of the nucleus of the lateral olfactory tract is essential for the normal functioning of the olfactory system. Brain Struct Funct 222, 3615-3637

Vochteloo, Koolhaas, (1987). Medial amygdala lesions in male rats reduce aggressive behavior: interference with experience. Physiology and Behavior, 41(2):99-102

Walker, David L., Donna J. Toufexis, and Michael Davis. "Role of the bed nucleus of the stria terminalis versus the amygdala in fear, stress, and anxiety." European journal of pharmacology463.1-3 (2003): 199216.

Wang Li, Zhang Zhijian, Chen Jiacheng, Manyande Anne, Haddad Rafi, Liu Qing, Xu Fuqiang, (2020). Cell-Type-Specific Whole-Brain Direct Inputs to the Anterior and Posterior Piriform Cortex. Frontiers in Neural Circuits, 14:4

Wang, Q, et al., (2020). The Allen Mouse Brain Common Coordinate Framework: A 3D Reference Atlas. Cell, 181(4):936-53.E20

Watabe-Uchida M, Zhu L, Ogawa SK, Vamanrao A, Uchida N. Whole-brain mapping of direct inputs to midbrain dopamine neurons. Neuron. 2012 Jun 7;74(5):858-73. doi: 10.1016/j.neuron.2012.03.017. PMID: 22681690.

Wickersham, I. R., Finke, S., Conzelmann, K. K., \& Callaway, E. M. (2007). Retrograde neuronal tracing with a deletion-mutant rabies virus. Nature methods, 4(1), 47-49.

Wickersham, Lyon, Barnard, RJO, Cozelmann, Young, Callaway, E.M., (2007). Monosynaptic Restriction of Transsynaptic Tracing from Single, Genetically Targeted Neurons. Neurotechnique, 53(5):639-47 
924 Winans SS, Scalia F. Amygdaloid nucleus: new afferent input from the vomeronasal organ. Science.

9251970 Oct 16;170(3955):330-2. doi: 10.1126/science.170.3955.330. PMID: 5460037.

Wu, M, Manoli, D, Fraser, E, Coats, J, Tollkuhn, J., Honda, S.I., Harada, N., Shah, N, (2009). Estrogen Masculinizes Neural Pathways and Sex-Specific Behaviors. Cell, 139(1):61-72.

Yamamoto, R., Ahmed, N., Ito, T., Gungor, N. Z., \& Pare, D. (2018). Optogenetic Study of Anterior BNST and Basomedial Amygdala Projections to the Ventromedial Hypothalamus. eNeuro, 5(3), ENEURO.020418.2018.

Yang, Y., Wang Jian-Zhi, (2017). From Structure to Behavior in Basolateral Amygdala-Hippocampus Circuits. Frontiers in Neural Circuits, 11:86

Yang, Chiang, Grag, Unger, Wells, Shah, N.M., (2013). Sexually Dimorphic Neurons in the Ventromedial Hypothalamus Govern Mating in Both Sexes and Aggression in Males. Cell, 153(4):896-909

Yao, S, Bergan, J, Lanjuin, A, Dulac, C, (2017). Oxytocin signaling in the medial amygdala is required for sex discrimination of social cues. eLife 2017;6:e31373.

Zhan and Luo, (2010). Diverse Patterns of Odor Representation by Neurons in the Anterior Piriform Cortex of Awake Mice. The Journal of Neuroscience, 30(49): 16662-72

Zikopoulos, Barbas, (2012). Pathways for Emotions and Attention Converge on the Thalamic Reticular Nucleus in Primates. Journal of neuroscience, 32(15):5338-50 


\section{Table Captions}

Table 1: Ipsilateral bias across brain regions. The average percentage of cells, as well as the absolute count of cells, are shown for each region on the contralateral hemisphere to the injection site.

Table 2: Hypothalamic inputs to aromatase+ cells in the MeA. The hypothalamic regions of the aromatase-expressing MeA cells. The top row describes course hypothalamic àMeAarom+. Each column enumerates the sub-regions in each respective course region àMeAarom+ cells from the top row.

Table 3: Statistical analysis of observed input cells across brain regions. The average percentage of input cells observed in each brain region and the results of two t-tests performed on percentage of cells in each region. 1) T-tests performed to determine if there is any significant input from each brain region. 2) T-tests performed to determine if the input from a given region is larger than expected assuming inputs directly proportional to region volume. The percentage of brain area that each brain region encompasses is also presented for this analysis. Degrees of freedom and standard deviation for each test are also provided.

Table 4: Statistical analysis of sex differences in major input regions. The average percentage of input cells observed in each region for males (mean column 2; SEM column 3) and females (mean column 4; SEM column 5). The p-value obtained from performing a posthoc permutation test comparing the percentage of inputs for each region in male versus female mice (column 6). Posthoc analyses followed an ANOVA with repeated samples. 


\section{Figure Captions}

Figure 1. Identifying aromatase-expressing (arom+) neurons in the mouse brain. A) The aromatase enzyme mediates the conversion of testosterone into estradiol. B) Crossing aromatase-cre and $\mathrm{A} 19$ tdTomato reporter mice ensures that the tdTomato fluorescent protein is coexpressed with aromatase. C) A large population of aromatase-expressing cells is present in the posterodorsal MeA [arrow in (1) horizontal, (2) sagittal and (3) coronal view.

Figure 2. Mapping the inputome of arom+ neurons in the MeA. A) Conditional AAV vectors were injected in arom-Cre mice to express TVA, RG, and mCherry in arom+ neurons of the MeA. B) Ten days after AAV injection, G-deleted rabies (GFP) was injected at the same stereotaxic coordinates to infect arom+ 'starter neurons'; followed by 7 days for retrograde movement of rabies from arom+ neurons in the MeA to upstream neurons. C) Infected 'starter neurons' in the MeApd identified by simultaneous tdtomato and eGFP expression. D) The number of neurons labeled by GFP injection outside the MeA scales linearly with the number of 'starter neurons' infected in the MeApd. E) View through the dorsal aspect of the brain showing location of 'starter neurons' (red) and inputome (green) demonstrating that input cells are almost entirely ipsilateral to the injection site (see graph). F) Lateral view of same showing that most inputs originate from subcortical regions.

Figure 3. Input cell numbers vary across subcortical brain regions. A sampling of input cells observed in one individual after alignment to the Allen Brain Atlas demonstrates that inputs can be localized to subcortical regions brain-wide and that input cell density varies among brain regions. A) Ventral tegmental area (VTA). B) Bed nucleus of the stria terminalis (BNST). C) Hippocampal formation (HPF). D) Medial preoptic area (MPOA). E) Ventromedial hypothalamic nucleus (VMH). F) Caudoputamen (CP). G) Central amygdalar nucleus (CeA). H) Accessory olfactory bulb (AOB). Each scale bar is $500 \mu \mathrm{m}$.

Figure 4. Coarse population count of the MeA arom+ inputome. Each input cell was assigned to one of 10 non-overlapping regions spanning the full volume of the reference brain. Defined regions are: cerebral nuclei (CNU), hypothalamus (H), Hippocampal formation (HPF), Cortical subplate (CTXsp), Olfactory areas (OF), Thalamus (TH), Midbrain (MB), Isocortex, Hindbrain (HB), and Cerebellum (CB). A) Graph showing the percentage of the average total observed input neurons found in each brain region relative to the volume of that brain region shows that some regions have either much higher or much lower density of inputs than would be expected if the cells were evenly distributed across the brain (straight line). B) Percentages of the average total number of input cells found in each defined brain region. C) Pie chart illustrating the proportions of inputome cells found in eight brain regions. Figures 4-1, 4-2, 4-3, and 4-4 are supporting Figure 4. 
1068

Figure 5. MeA arom+ inputome originating in the cerebral nuclei (CNU). A) Left, a pie chart indicates the fraction of all labeled neurons in the $\mathrm{CNU}$ and the brain atlas depicts the position of hypothalamic regions. Right, bar graph depicts the percentages of input cells found in the striatum (STR) and pallidum (PAL) relative to the whole-brain inputome; pie chart shows the relative proportion of those cells within the cerebral nuclei alone. B) Locations of input neurons within the striatum: line graph shows relative density of cells in subregions of the striatum, bar graph shows relative distribution of inputs relative to the whole-brain inputome; pie chart shows the relative proportion of those cells within parts of the striatum. Regions: Medial amygdala (MEA), Central amygdala (CEA), Caudoputamen (CP), Intercalcated amygdalar nucleus (IA), Anterior amygdalar area (AAA), Nucleus accumbens (ACB), Lateral septal nucleus (LS), Fundus of striatum (FS), Olfactory tubercle (OT), Septofimbrial nucleus (SF), Bed nucleus of the accessory olfactory tract (BA), Septohippocampal nucleus (SH). C) Locations of input neurons within the pallidum: bar graph shows relative distribution of inputs relative to the whole-brain inputome; pie chart shows the relative proportion of those cells within parts of the pallidum. Regions: External globus pallidus (GPe), Substantia innominata (SI), Internal globus pallidus (GPi), Bed nucleus of the stria terminalis (BNST), Diagonal band nucleus (NDB), Magnocellular nucleus (MA), Medial septal nucleus (MS), Triangular nucleus of septum (TRS). Figure 5-1 is supporting Figure 5.

Figure 6. MeA arom+ inputome originating in the hypothalamus (HY). A) Left, a pie chart indicates the fraction of all labeled neurons in the hypothalmus and the brain atlas depicts the position of hypothalamic regions. Right, bar graph depicts the percentages of input cells found in the five subregions of the hypothalamus relative to the whole-brain inputome; pie chart shows the relative proportion of those cells within those hypothalamic regions. Regions: Lateral zone (LZ), Medial zone (MEZ), Periventricular region (PVR), Periventricular zone (PVZ), Median eminence (ME). B) Locations of input neurons within the lateral zone of the hypothalamus: bar graph shows relative distribution of inputs relative to the whole-brain inputome; pie chart shows the relative proportion of those cells within parts of the medial zone. Regions: Lateral hypothalamic area (LHA), Tuberal nucleus (TU), Zona incerta (ZI), Lateral preoptic area (LPO), Subthalamic nucleus (STN), Parasubthalamic nucleus (PSTN), Retrochiasmatic area (RCH), Perifornical nucleus (PeF), Preparasubthalamic nucleus (PST). C. Locations of input neurons within the medial zone of the hypothalamus: bar graph shows relative distribution of inputs relative to the whole-brain inputome; pie chart shows the relative proportion of those cells within parts of the medial zone. Regions: Ventromedial hypothalamic nucleus (VMH), ventral premammillary nucleus (PMv), medial mammillary nucleus (MM), Medial preoptic nucleus (MPN), dorsal premammillary nucleus (PMd), Anterior hypothalamic nucleus (AHN), Posterior hypothalamic nucleus (PH), Tuberomammillary nucleus (TM), Supramammillary nucleus (SUM), Paraventricular hypothalamic nucleus (PVHd), Lateral mammillary nucleus (LM). Figure 6-1 is supporting Figure 6.

Figure 7. MeA arom+ inputome originating in the hippocampal formation (HPF) and the cortical subplate (CTXsp). A) Left, a pie chart indicates the fraction of all labeled neurons in the hippocampus and the brain atlas depicts the position of hippocampal regions. Right, bar graph depicts the percentages of input cells found in the regions of the hippocampal formation relative to the whole-brain inputome; pie chart shows the relative proportion of those cells within those regions. The lower inset image illustrates that input cells are located primarily in ventral portions of the HPF. Regions: Field CA1 (CA1), Field CA2 (CA2), Field CA3 (CA3), Entorhinal area (ENT), Hippocampalamygdalar transition area (HATA), Prosubiculum (ProS), Dentate gyrus (DG), Presubiculum (PRE), Subiculum (SUB), Area prostriata (APr), Postsubiculum (POST), Parasubiculum (PAR), Induseum 
griseum (IG), Fasciola cinerea (FC). B) Left, a pie chart indicates the fraction of all labeled neurons in the cortical subplate and the brain atlas depicts the position of cortical subplate regions. Right, bar graph depicts the percentages of input cells found in the regions of the cortical subplate relative to the whole-brain inputome; pie chart shows the relative proportion of those cells within those regions. Regions: Posterior amygdalar nucleus (PA), Anterior basomedial amygdalar nucleus (BMAa), Posterior basomedial amygdalar nucleus (BMAp), Anterior basolateral amygdalar nucleus (BLAa), Ventral basolateral amygdalar nucleus (BLAv), Posterior basolateral amygdalar nucleus (BLAp), Lateral amygdalar nucleus (LA), Ventral endopiriform nucleus (EPv), Dorsal endopiriform nucleus (EPd), Claustrum (CLA). Figure 7-1 is supporting Figure 7.

Figure 8. MeA arom+ inputome originating in the olfactory areas (OLF) and the thalamus (TH). A) Left, a pie chart indicates the fraction of all labeled neurons in olfactory areas and the brain atlas depicts the position of olfactory areas. Right, bar graph depicts the percentages of input cells found in subregions of the olfactory area relative to the whole-brain inputome; pie chart shows the relative proportion of those cells within those regions. Regions: Cortical amygdalar area (COA), piriform area (PIR), Accessory olfactory bulb (AOB), Piriform-amygdalar area (PAA), Postpiriform transition area (TR), Nucleus of the lateral olfactory tract (NLOT), Main olfactory bulb (MOB), Taenia tecta (TT), Anterior olfactory nucleus (AON), Dorsal peduncular area (DP). B) Left, a pie chart indicates the fraction of all labeled neurons in the thalamus and the brain atlas depicts the position of thalamic regions. Right, bar graph depicts the percentages of input cells found in subregions of the thalamus relative to the whole-brain inputome; pie chart shows the relative proportion of those cells within those regions. Regions: Reticular nucleus (RT), Ventral group of the dorsal thalamus (VENT), Dorsal geniculate group (GENd), Midline group of the dorsal thalamus (MTN), Ventral geniculate group (GENv), Intralaminar nuclei of the dorsal thalamus (ILM), Peripeduncular nucleus (PP), Lateral group of the dorsal thalamus (LAT), Medial group of the dorsal thalamus (MED), Epithalamus (EPI), Anterior group of the dorsal thalamus (ATN), Subparafascicular area (SPA), Subparafascicular nucleus (SPF). Figure $8-1$ is supporting Figure 8.

Figure 9. Sex differences in the inputome of aromatase-expressing cells in the MeA. The average percentage of synaptic inputs to aromatase-expressing cells in the MeA compared for each region in males and females. Only regions that provide $0.5 \%$ of the overall input are shown and regions identified as having a statistically significant sex difference are shown in color $(p<0.05$ : Permutation post-hoc test following ANOVA with repeated measures on 'sex','region', and 'individual'). These regions include field CA1 (CA1), the accessory olfactory bulb (AOB), piriform area (PIR), piriform-amygdalar area (PAA), postpiriform transition area (TA), basolateral amygdalar nucleus- anterior (BLAa), basolateral amygdalar nucleus-ventral (BLAv), diagonal band nucleus (NDB), lateral preoptic area (LPO), and ventral premammillary nucleus (PMv). Regions lying above the line of unity represent a male bias and neurons lying to the right of the unity line indicate a female bias. Error bars indicate the standard errors of the mean (male: vertical; female: horizontal) for each region. Figure $9-1$ is supporting Figure 9. 
Figure 10. The inputome of aromatase-expressing cells in the MeA. A) Illustration showing the relative locations of the major inputs to the aromatase-expressing MeA cells throughout the brain as defined by our data. Major regions are shaded to demonstrate that the included regions were analyzed in groups and are considered components of a larger analysis region. These inputs were selected based on their relative abundance in the data and behavioral significance as represented in previous literature. B) A Venn diagram showing the relative distribution of the regions displayed in A sorted by their reported roles in the production or modulation of Memory, Parenting and Aggression, Metabolism, Socio-sexual Behaviors, and Fear and Anxiety behaviors.

\section{Supplementary Figure Captions}

Figure 4-1. Input cell density in regions brain-wide. Percentage of input cells in each coarse region identified in the Allen Brain Atlas normalized to the volume of that region to determine cell densities in each region are presented as A) graph and B) pie chart. The densest concentration of input cells is found in the cortical subplate (CTXsp).

Figure 4-2. MeA arom+ inputome originating in the midbrain (MB). A) Bar graph depicts the percentages of input cells found in major divisions of the midbrain relative to the whole-brain inputome; pie chart shows the relative proportion of those cells within those regions. Regions: motorrelated midbrain regions (MBmot), sensory-related midbrain regions (MBsec), behavior-related midbrain regions (MBsta). B) Inputs from motor-related midbrain regions: Bar graph depicts the percentages of input cells found in major divisions of these regions relative to the whole-brain inputome; pie chart shows the relative proportion of input cells within those regions. Regions: Midbrain reticular nucleus (MRN), Superior colliculus [motor] (SCm), Ventral tegmental area (VTA), Substantia nigra (SNr), Periaqueductal gray (PAG), Lateral terminal nucleus of accessory optic tract (LT), Cuneiform nucleus (CUN), Red nucleus (RN), Retrorubal area of midbrain reticular nucleus (RR), Paratrochlar nucleus (Pa4), Pretectal region (PRT), Medial terminal nucleus of accessory optic tract (MT), Dorsal terminal nucleus of accessory optic tract (DT), Anterior tegmental nucleus (AT), Ventral tegmental nucleus (VTN), Troclear nucleus (IV), Edinger-Westphal nucleus (EW), Medial accessory oculomotor nucleus (MA3), Oculomotor nucleus (III), Paranigral nucleus (PN). C) Inputs from sensory-related midbrain regions: Bar graph depicts the percentages of input cells found in major divisions of these regions relative to the whole-brain inputome; pie chart shows the relative proportion of input cells within those regions. Regions: Inferior colliculus (IC), Brachium of inferior colliculus (NB), Subcommissural organ (SCO), Midbrain trigeminal nucleus (MEV), Parabigeminal nucleus (PBG), Nucleus sagulum (SAG), Superior colliculus [sensory] (SCs).

${ }^{* *}$ Less than $0.01 \%$ of MeA arom+ inputs originate in behavior-related midbrain area; most of those cells are found in the Dorsal raphe nucleus (DR), Pedunculopontine nucleus (PPN), and Interpeduncular nucleus (IPN). 
Figure 4-3. MeA arom+ inputome originating in the Hindbrain (HB). A) Whole hindbrain: Bar graph depicts the percentages of input cells found in major divisions of the hindbrain relative to the whole-brain inputome; pie chart shows the relative proportion of input cells within those regions. Regions: Motor-related medulla (MY-mot), Sensory-related medulla (MY-sen), Behavioral state medulla (MYsat), Motor-related pons (P-mot), Sensory-related pons (P-sen), Behavioral state pons (P-sat). B) Only one of the behavior-state portions of the medulla, the nucleus raphe magnus (RM) contains inputs to arom+ cells in the MeA. C) Bar graph depicts the percentages of input cells found in major divisions of the motor-related medulla relative to the whole-brain inputome; pie chart shows the relative proportion of input cells within those regions. Two regions of the motor-related medulla provide inputs: the Gigantocellular reticular nucleus (GRN) and the Facial motor nucleus (VII). D) The caudal part of the pontine reticular nucleus (PRNc) is the only part of the motor-related pons to provide inputs to arom+ MeA cells. E) In the sensory-related pons, inputs originate from the parabrachial nucleus (PB) and the nucleus of the lateral lemniscus (NLL).

Figure 4-4. MeA arom+ inputome originating in the Isocortex and the Cerebellum (CB). A) Bar graph depicts the percentages of input cells found in major divisions of the isocortex relative to the whole-brain inputome; pie chart shows the relative proportion of input cells within those regions. Regions: Secondary motor area (MOs), Agranular insular area (Al), Orbital area (ORB), Temporal association area (Tea), Ectorhinal area (ECT), Visceral area (VISC), Anterior cingulate area (ACA), Infralimbic area (ILA), Primary motor area (MOp), Supplementary somatosensory area (SSs), Gustatory areas (GU), Auditory areas (AUD), Visual areas (VIS), Primary somatosensory area (SSp), Prelimbic area (PL), Perirhinal area (PERI), Posterior parietal association areas (PTLp), Retrosplenal area (RSP). B) Inputs from the cerebellum are only found in the cerebellar cortex (CBX).

Figure 5-1. Input cell density in the cerebral nuclei (CNU). A) Input cell densities of constituent regions of the cerebral nuclei. B) Input cell densities of constituent regions in the striatum. C) Input cell densities of constituent regions in the pallidum. Results are presented as both bar and pie charts.

Figure 6-1. Input cell density in the hypothalamus (HY). A) Input cell densities of constituent regions in the hypothalamus. B) Input cell densities of constituent regions in the lateral zone of the hypothalamus. C) Input cell densities of constituent regions in the medial zone of the hypothalamus. Results are presented as both bar chart and pie charts.

Figure 7-1. Input cell density in the hippocampal formation (HPF) and the cortical subplate (CTXsp). A) Input cell densities of constituent regions in the hippocampal formation. B) Input cell densities of constituent regions in the cortical subplate. Results are presented as both bar and pie charts.

Figure 8-1. Input cell density in the olfactory areas (OLF) and the thalamus (TH). A) Input cell densities of constituent regions in the olfactory areas. B) Input cell densities of constituent regions in the thalamus. Results are presented as both bar and pie charts.

Figure 9-1. Comparison of sex differences in observed inputs to $\mathrm{MeA}^{\mathrm{Arom}+}$ Cells. A) Graph comparing the fraction of labeled neurons in the MeA, local to the injection site, in females and males. B) graph showing the d-prime values for sex differences in the regions presented in Figure 9. 
a

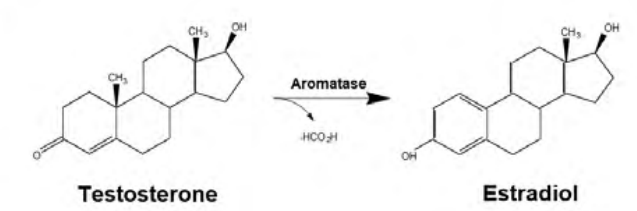

c1

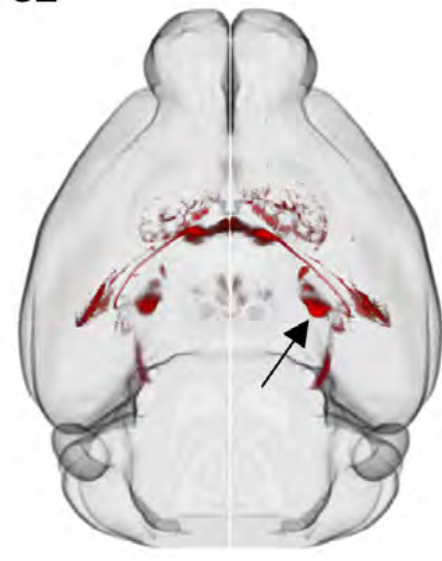

b

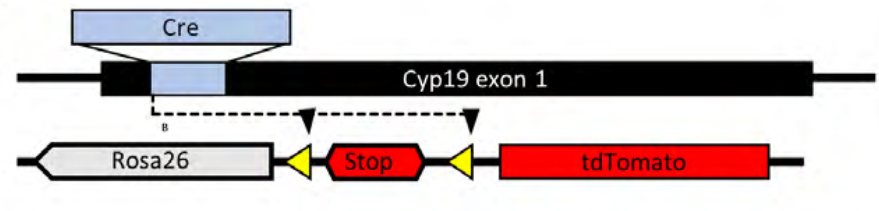

c3
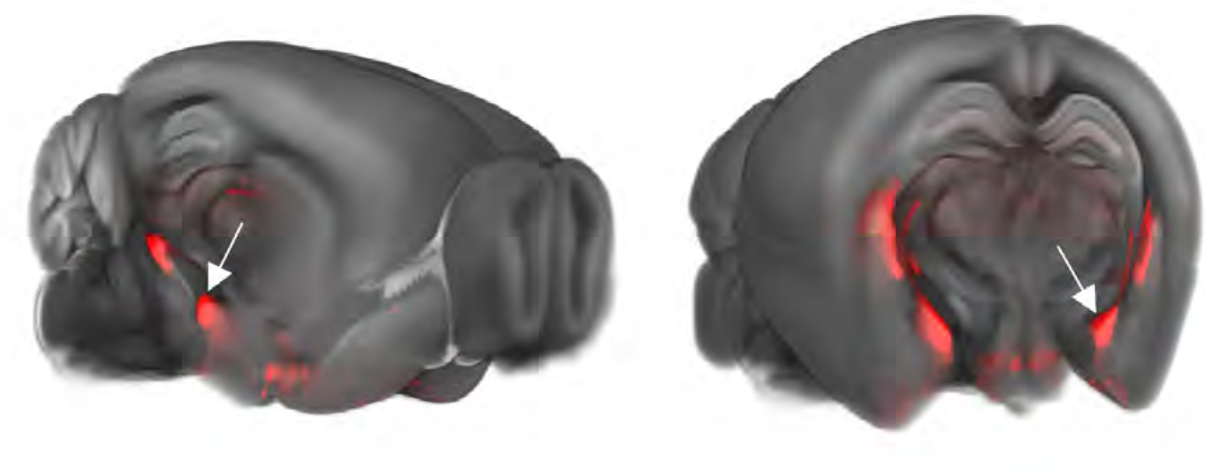
a
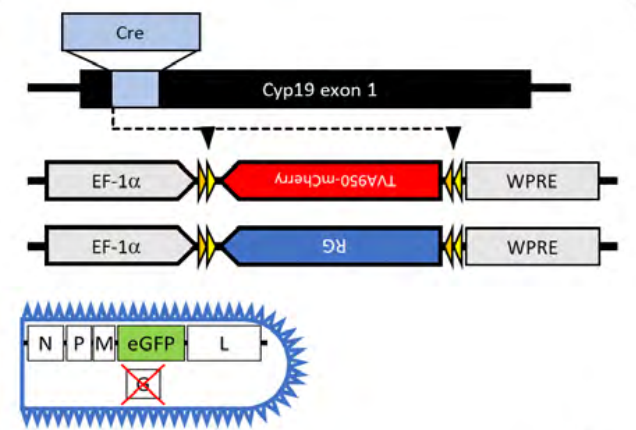

c1

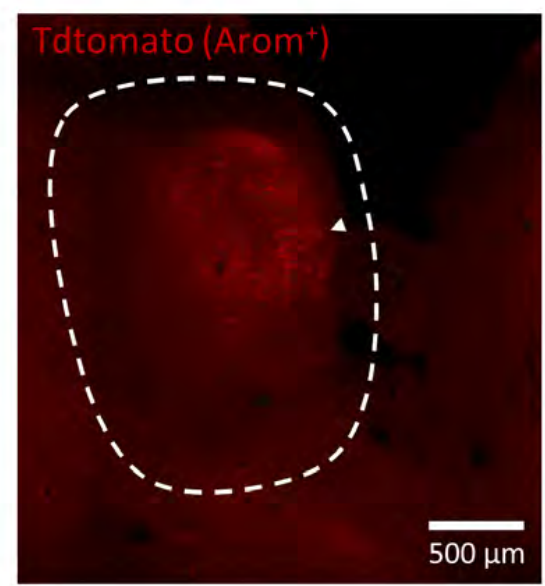

()

(u)

(u)

$<$

()

(1)

(d)

c2 b
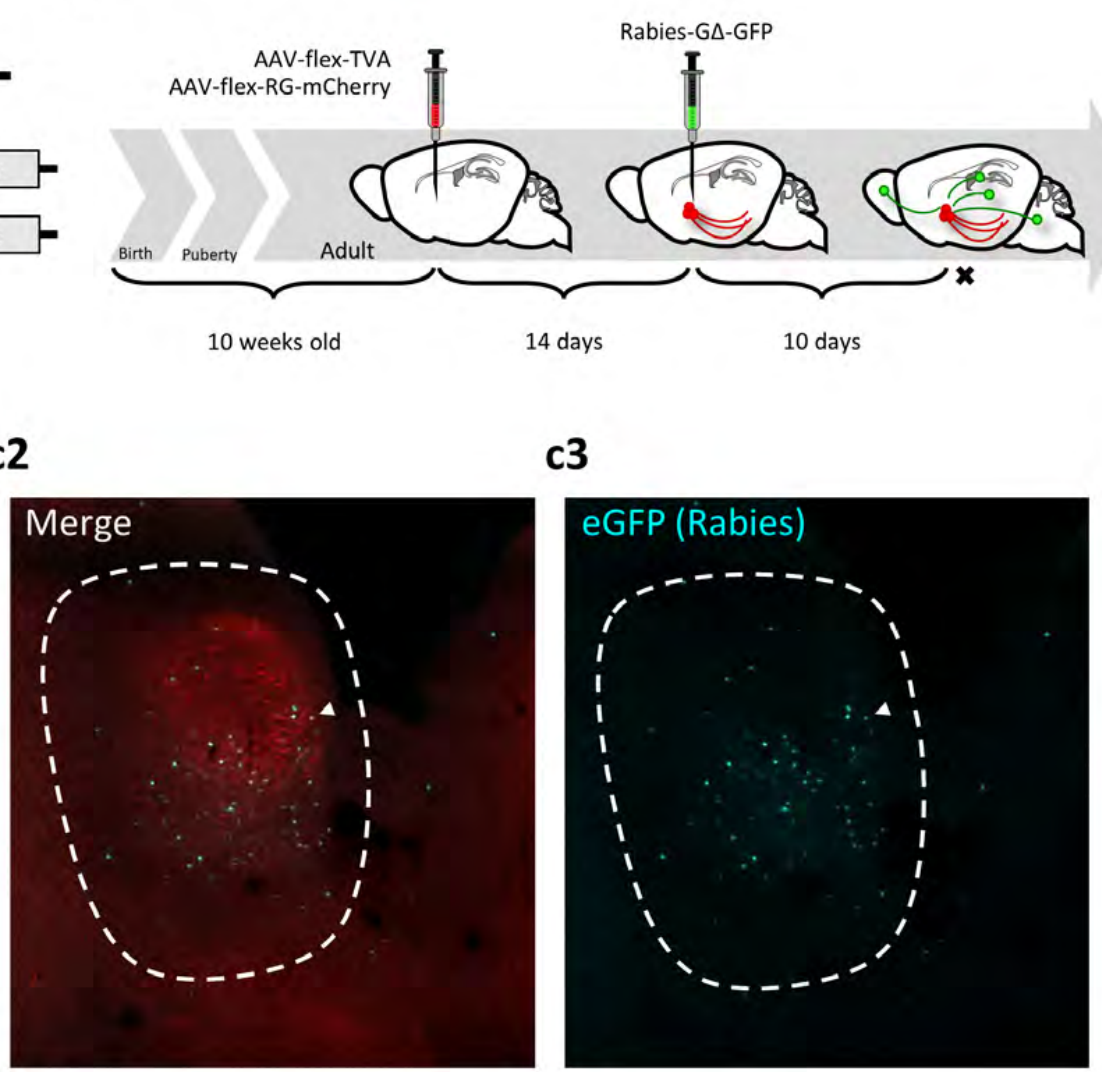

e
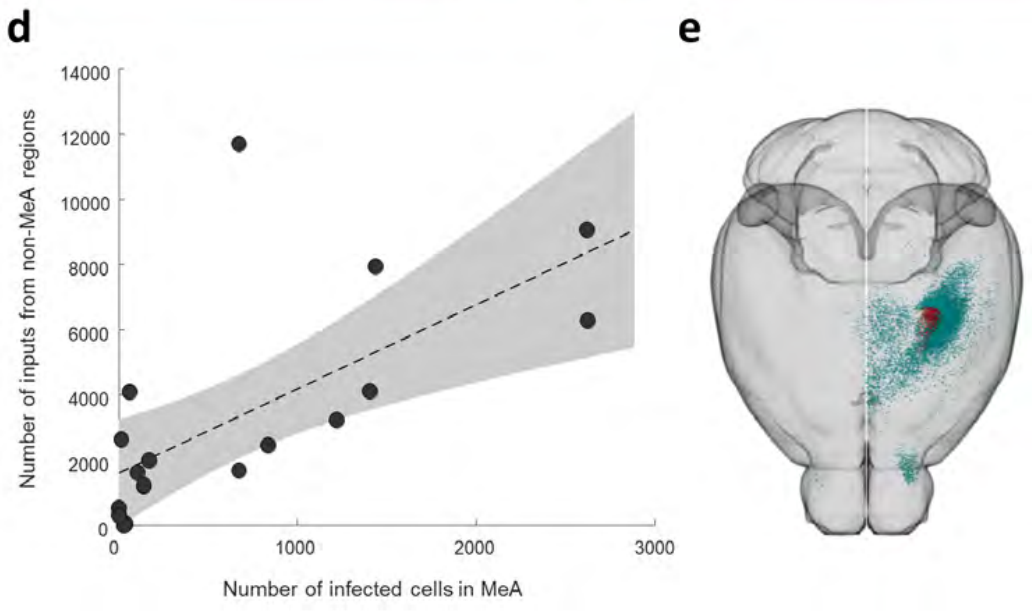

c3
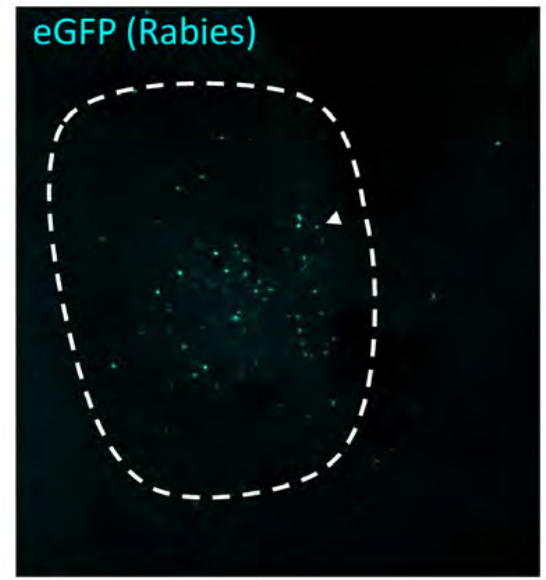

f

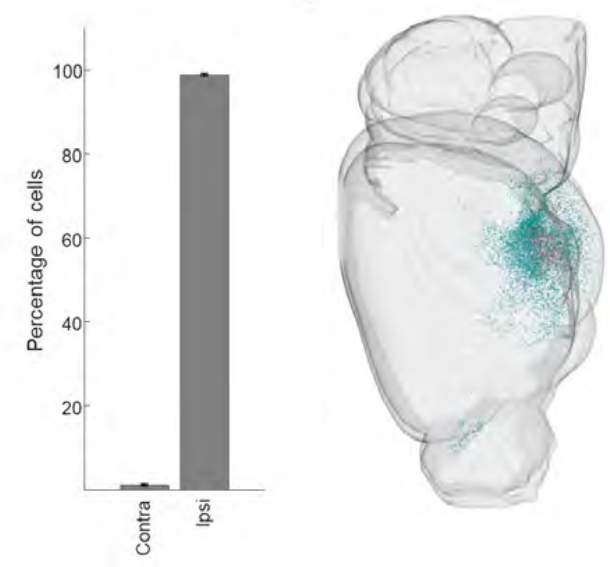




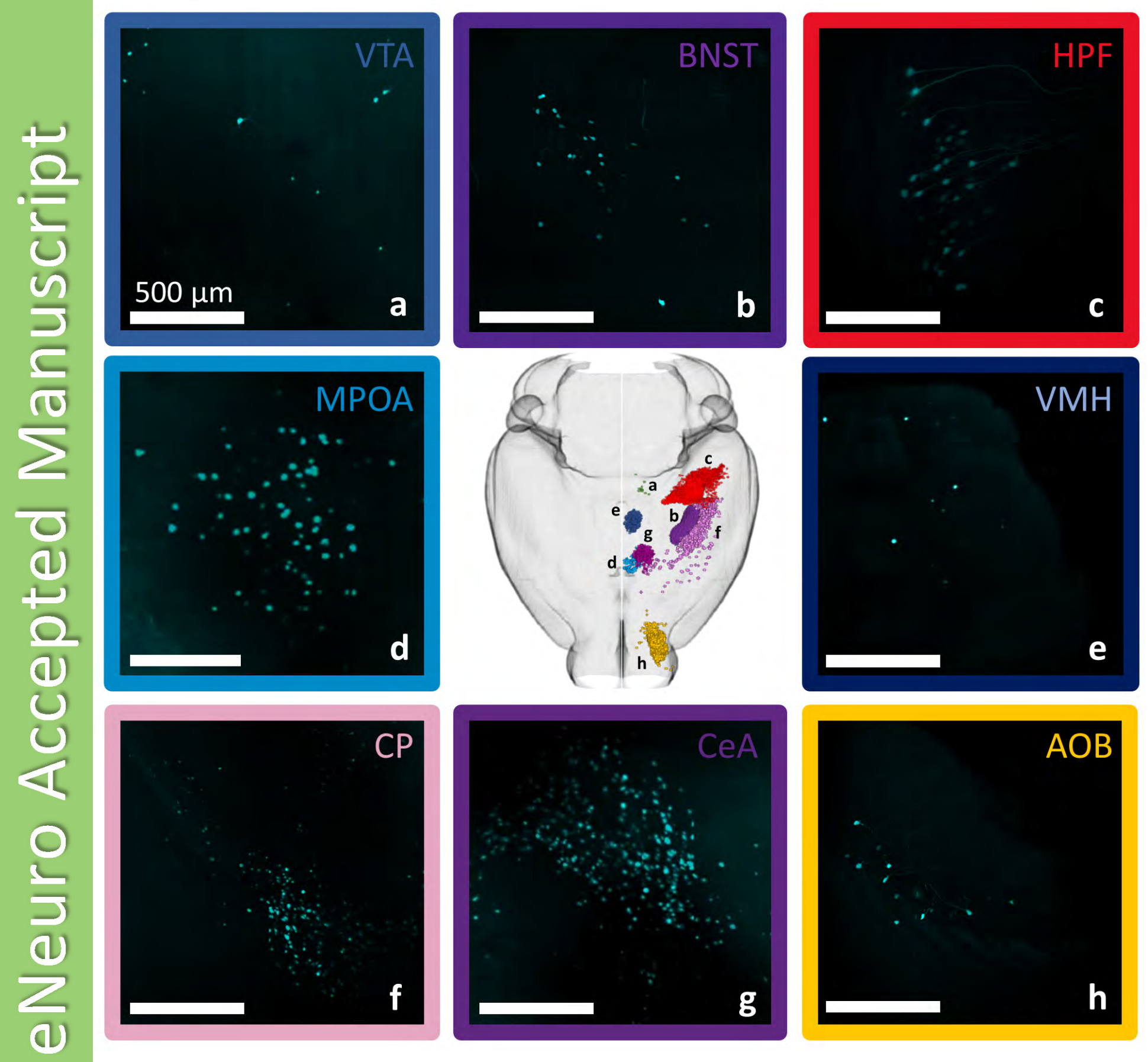




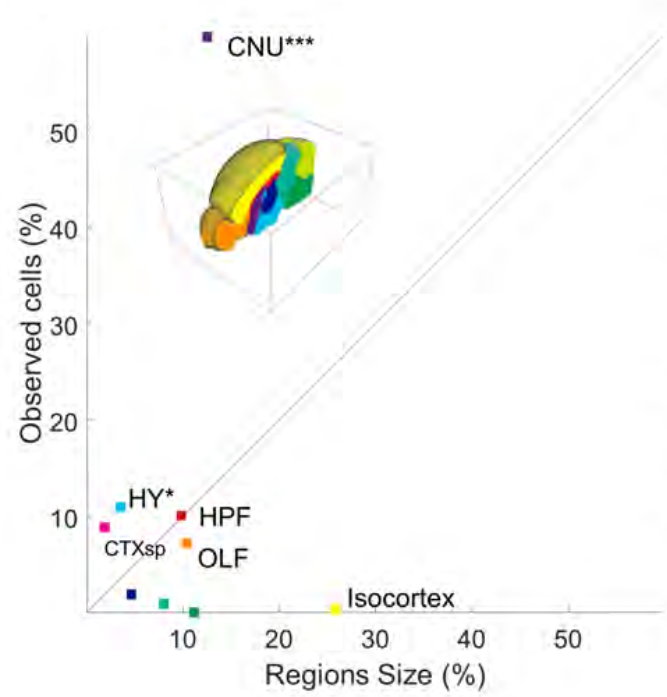

b

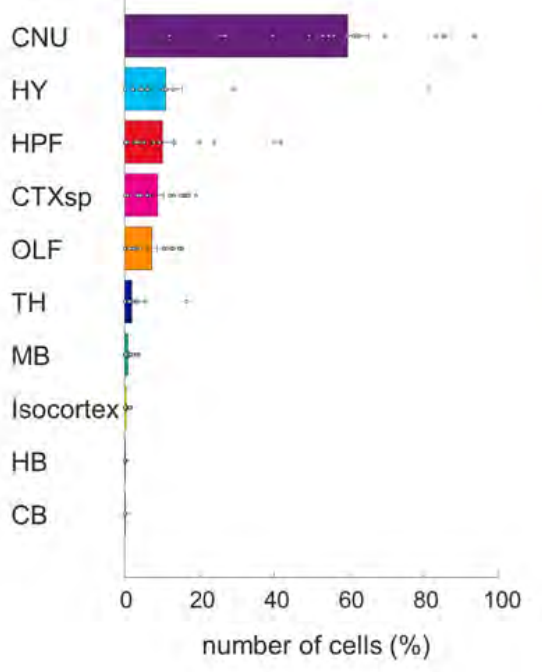

c

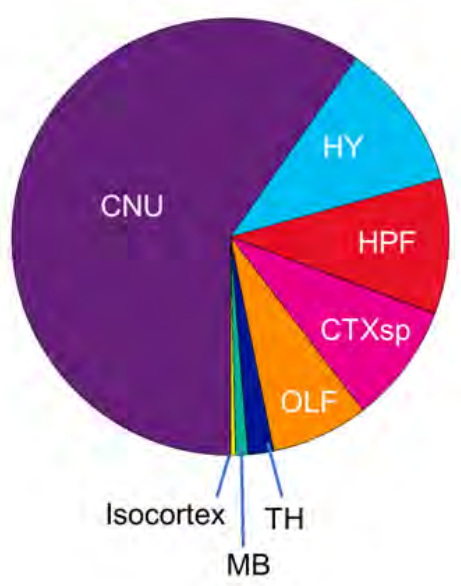


a Cerebral Nuclei (CNU)

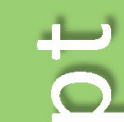

b Striatum (STR)
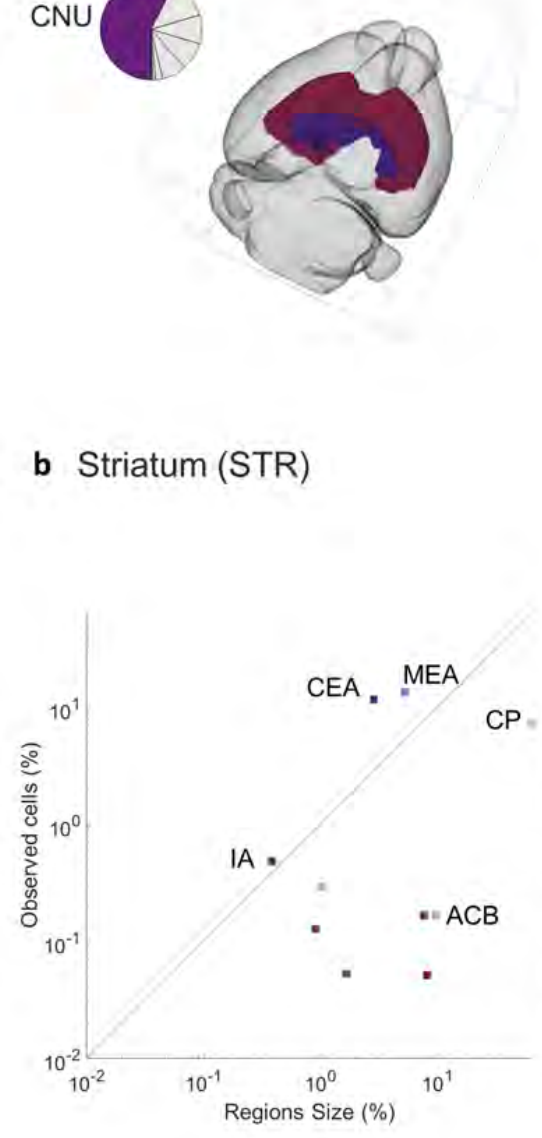

()

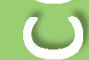

c Pallidum (PAL)
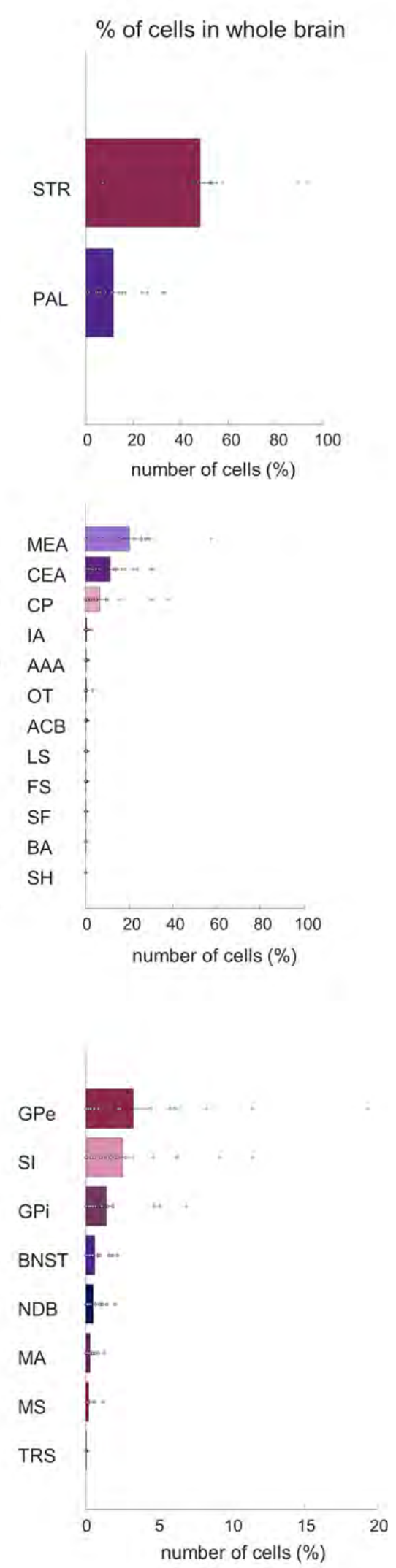

proportion of cells in region
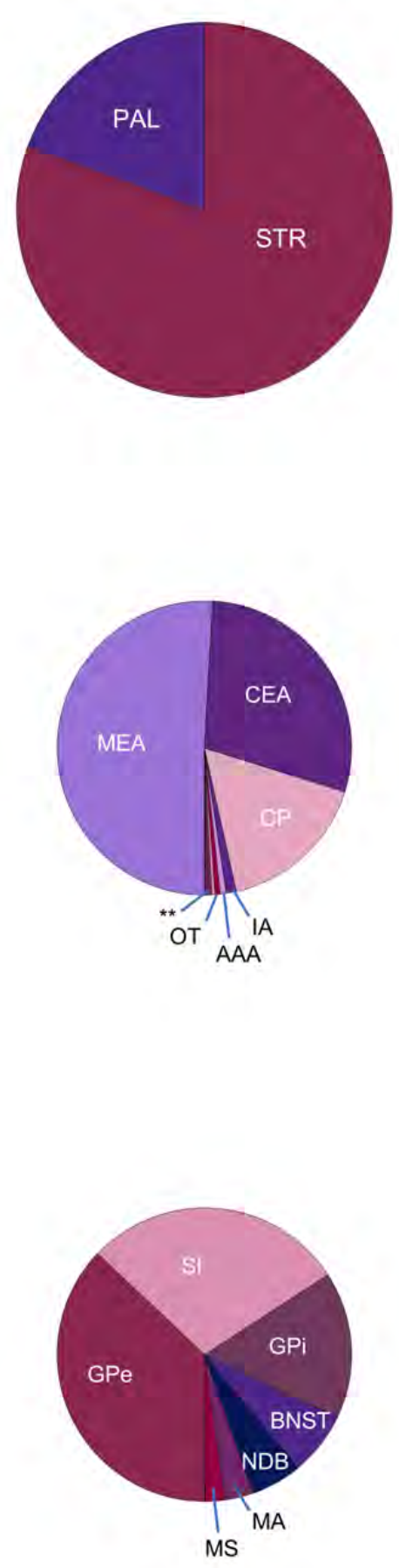
a Hypothalamus (HY)

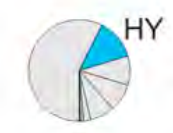

b Lateral zone (LZ)
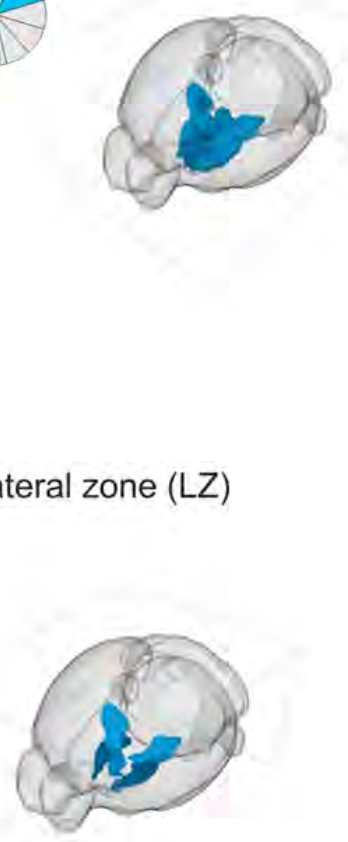

c Medial zone (MEZ)
$\%$ of cells in whole brain
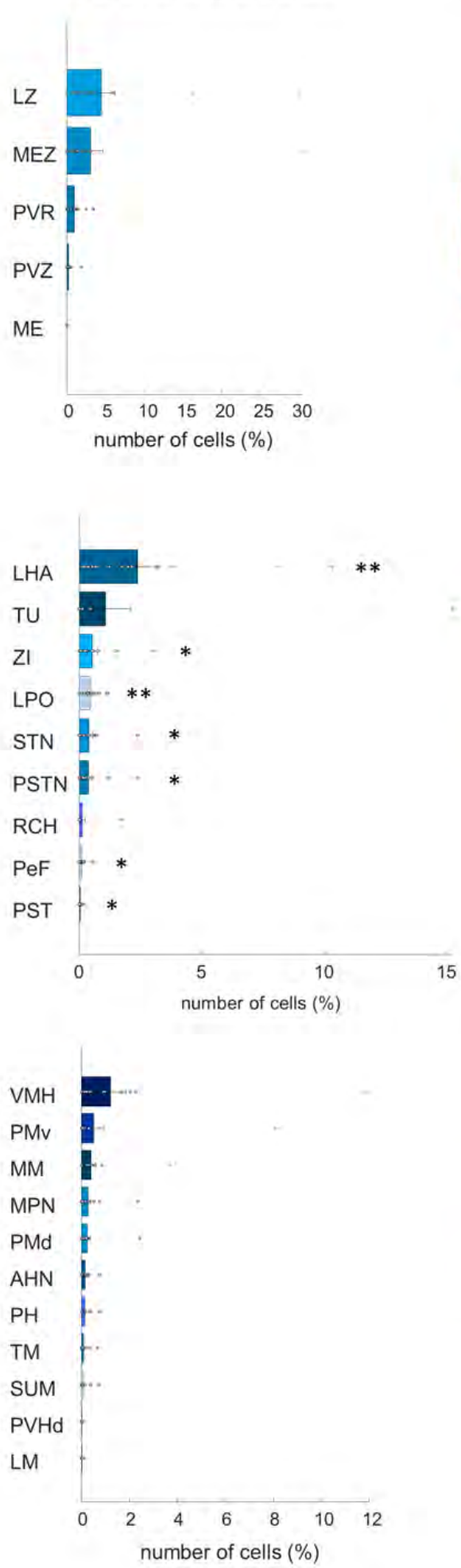

proportion of cells in region
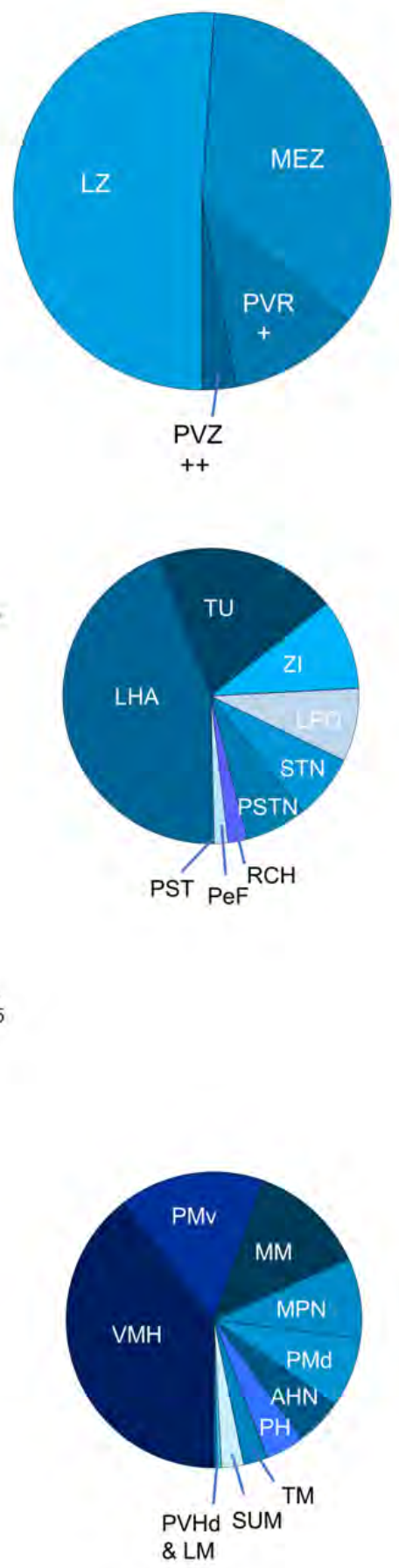

+ Periventricular region (PVR): Areas contain 1.1\% of MeA arom + inputs, most cells originate in the DMH and MPO, with smaller contributions from the AVP, PVp, and AVPV.

++ Periventricular zone (PVZ): Areas contain less than $0.5 \%$ of $\mathrm{MeA}$ arom+ inputs, most cells originate in the $\mathrm{ARH}$ and $\mathrm{PVi}$, with smaller contributions from the PVH and SO. 
a Hippocampal formation (HPF) \% of cells in whole brain
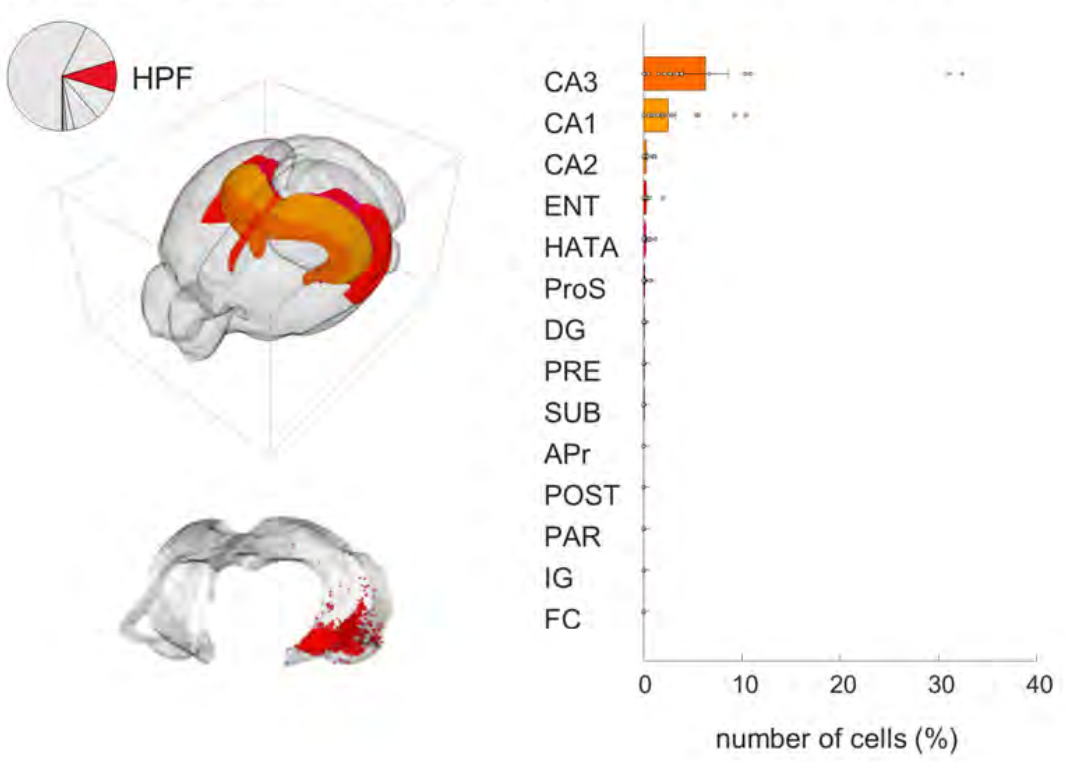

\section{(4)}

b Cortical subplate (CTXsp)
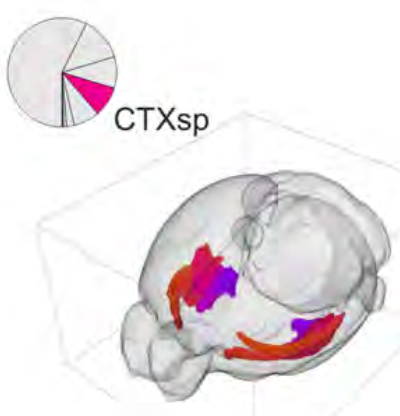

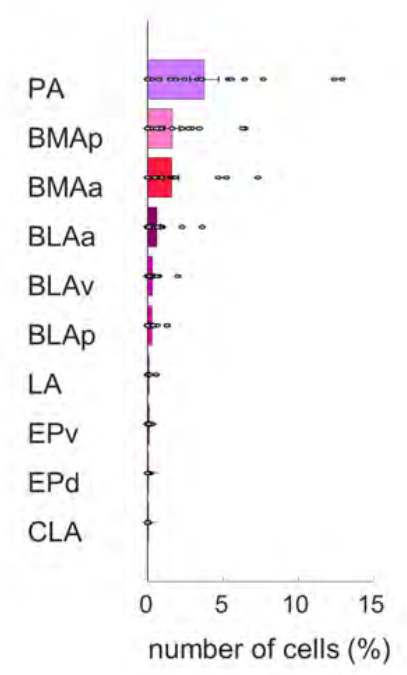

proportion of cells in region
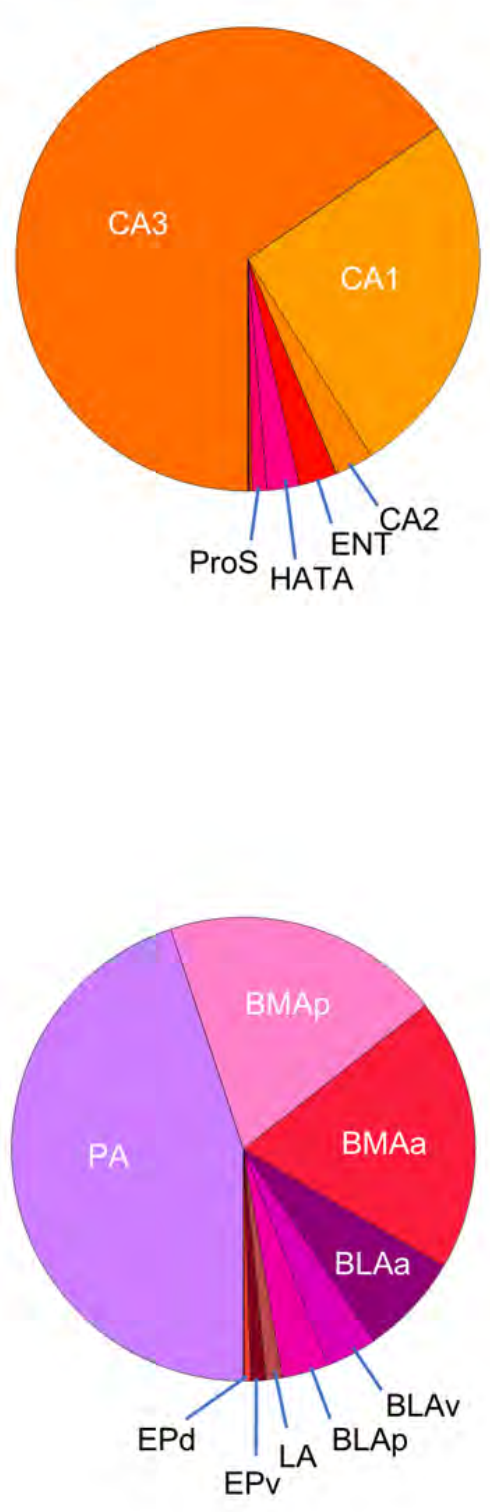
a Olfactory areas (OLF)

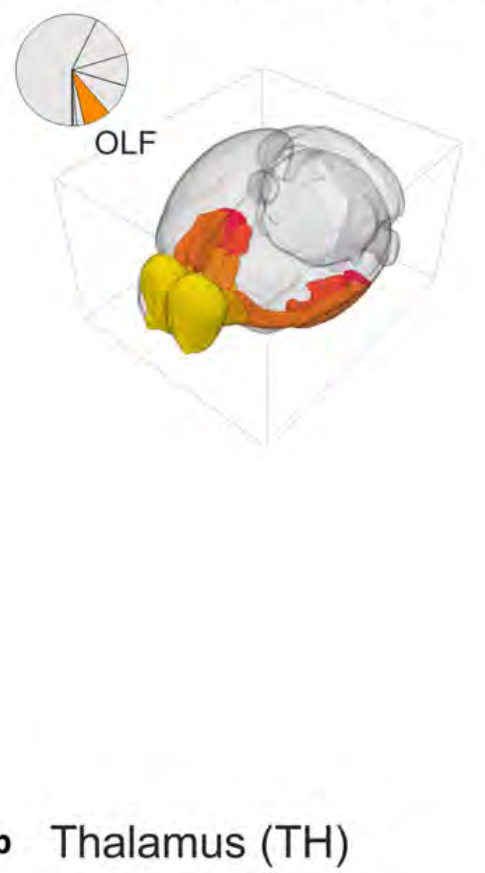

$\%$ of cells in whole brain

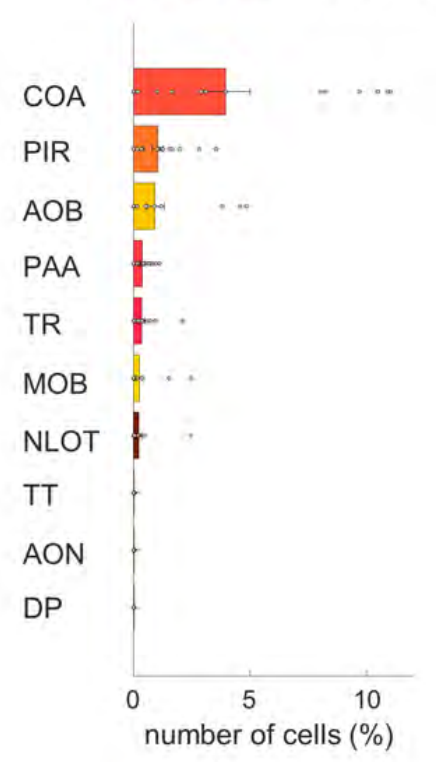

(i)

ㄴ)

2.

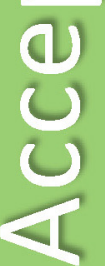

(2)

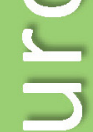

(a)

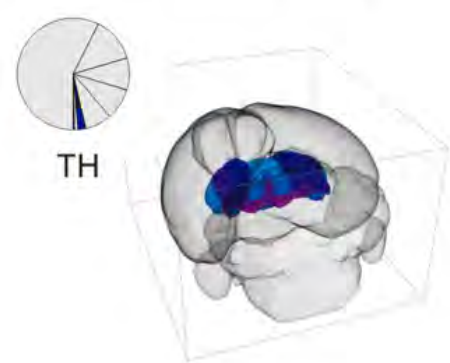

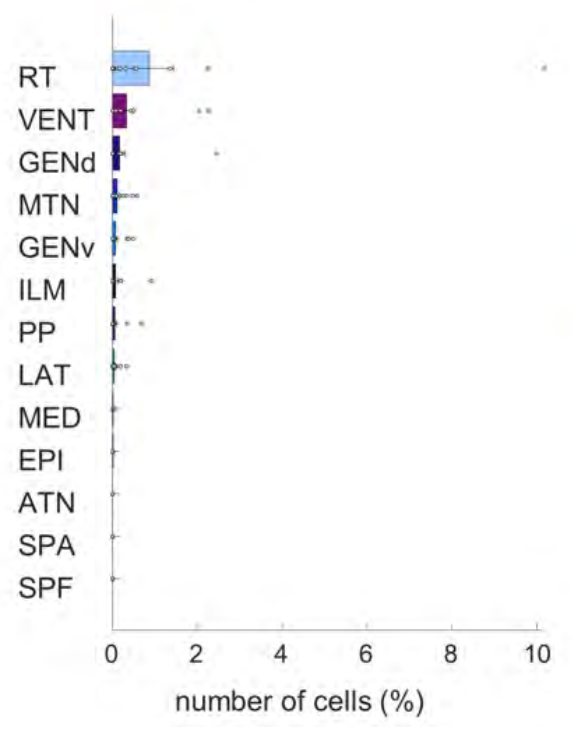

proportion of cells in region
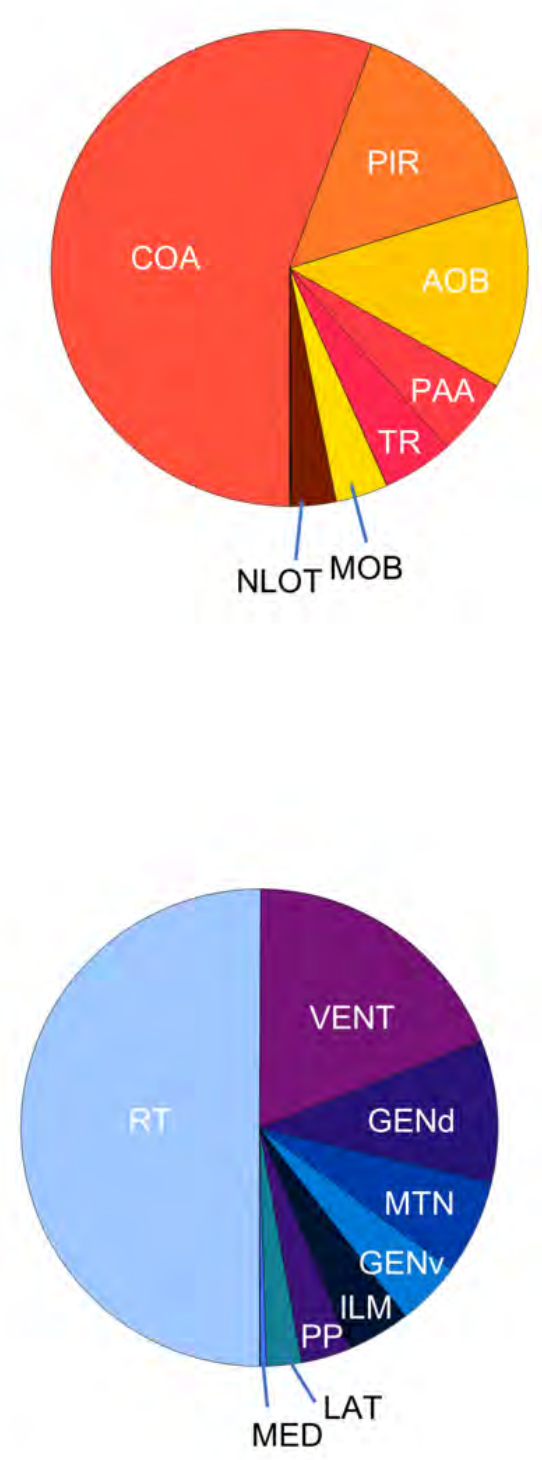


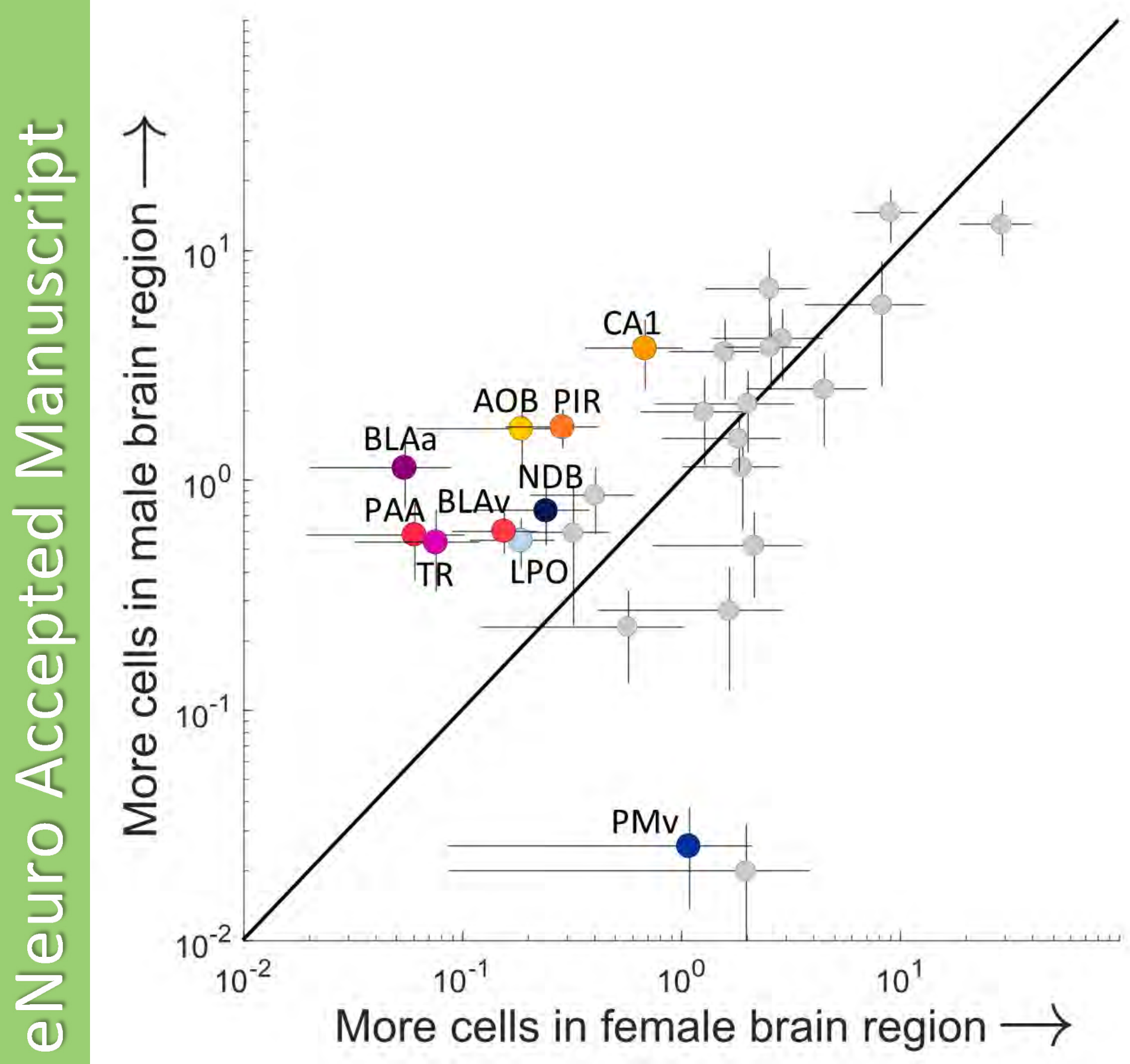




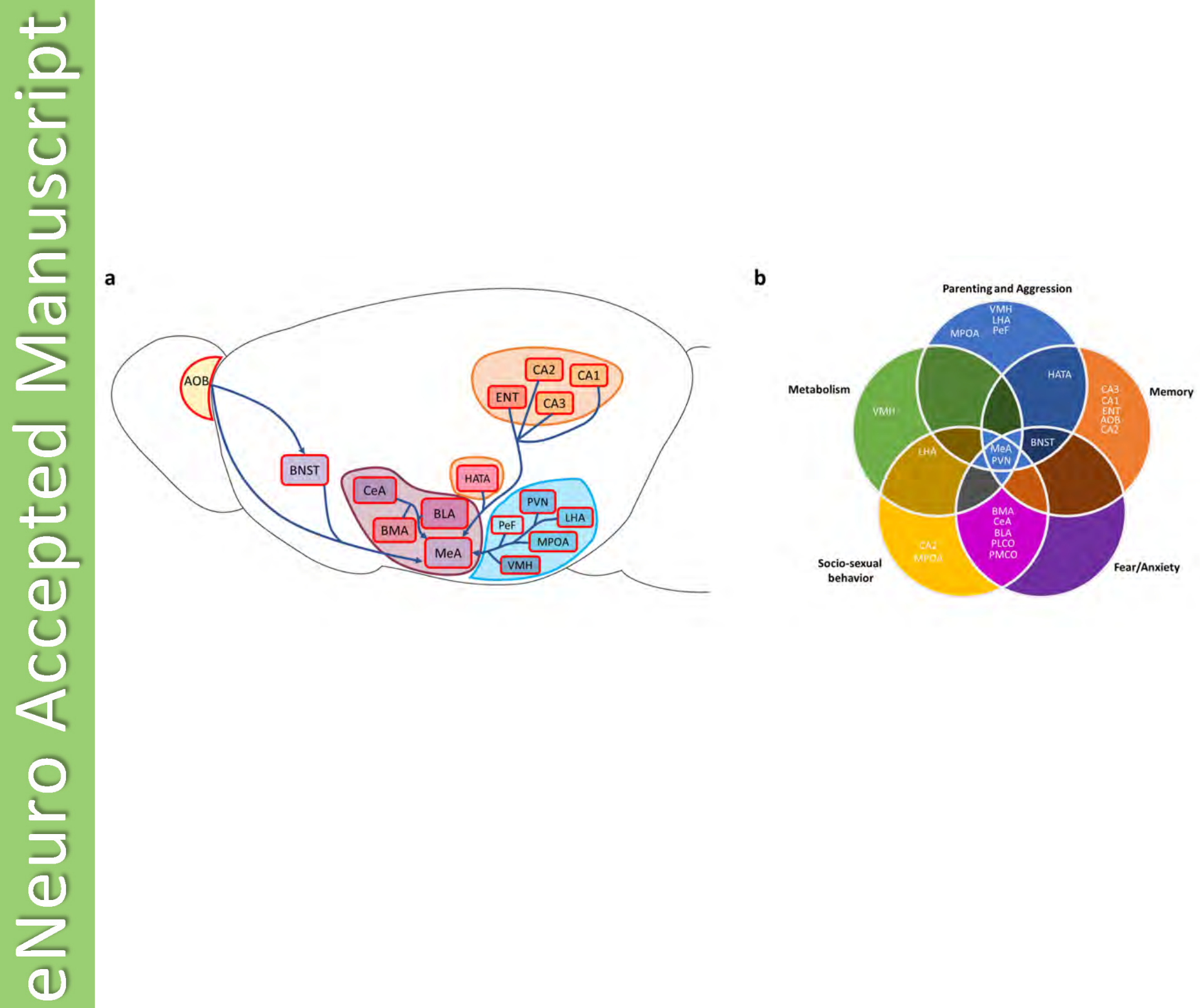


Region name

Inferior colliculus

Basic cell groups and regions

Anterior amygdalar area

Periventricular hypothalamic nucleus, anterior part

Anterior cingulate area

Paraventricular hypothalamic nucleus

Infralimbic area

Intralaminar nuclei of the dorsal thalamus

Nucleus accumbens

Paraventricular hypothalamic nucleus, descending division Lateral terminal nucleus of the accessory optic tract

Anterodorsal preoptic nucleus

Anterior hypothalamic nucleus

Agranular insular area

Interpeduncular nucleus

Periventricular hypothalamic nucleus, intermediate part

Periventricular hypothalamic nucleus, posterior part

Midbrain reticular nucleus

Lateral amygdalar nucleus

Periventricular hypothalamic nucleus, preoptic part

Lateral group of the dorsal thalamus

Accessory olfactory bulb

Anterior olfactory nucleus

Retrochiasmatic area

Lateral hypothalamic area

Nucleus raphe magnus

Lateral mammillary nucleus

Red nucleus

Arcuate hypothalamic nucleus

Lateral preoptic area

Lateral septal nucleus

Midbrain reticular nucleus, retrorubral area

Auditory areas

Reticular nucleus of the thalamus

Anteroventral preoptic nucleus

Anteroventral periventricular nucleus

Suprachiasmatic nucleus

Bed nucleus of the accessory olfactory tract

Superior colliculus, motor related

Magnocellular nucleus

Basolateral amygdalar nucleus, anterior part

Septofimbrial nucleus

Basolateral amygdalar nucleus, posterior part

Primary somatosensory area

Basomedial amygdalar nucleus, anterior part

Accessory supraoptic group

\begin{tabular}{|c|c|}
\hline Contra percentage & Total number \\
\hline 0 & 0 \\
\hline 0.770459457 & 82 \\
\hline 0 & 0 \\
\hline 85.71428571 & 6 \\
\hline 75 & 3 \\
\hline 6 & 3 \\
\hline 0 & 0 \\
\hline 2.631578947 & 1 \\
\hline 1.538461538 & 2 \\
\hline 0 & 0 \\
\hline 0 & 0 \\
\hline 4.347826087 & 1 \\
\hline 0 & 0 \\
\hline 3.389830508 & 2 \\
\hline 50 & 1 \\
\hline 48.35164835 & 44 \\
\hline 8.333333333 & 3 \\
\hline 6.666666667 & 1 \\
\hline 0 & 0 \\
\hline 22.91666667 & 11 \\
\hline 0 & 0 \\
\hline 0 & 0 \\
\hline 0 & 0 \\
\hline 0 & 0 \\
\hline 0.412654746 & 6 \\
\hline 50 & 1 \\
\hline 10 & 1 \\
\hline 0 & 0 \\
\hline 12.85714286 & 9 \\
\hline 1.308900524 & 5 \\
\hline 1.015228426 & 2 \\
\hline 0 & 0 \\
\hline 0 & 0 \\
\hline 0 & 0 \\
\hline 1.785714286 & 2 \\
\hline 6.25 & 5 \\
\hline 66.66666667 & 2 \\
\hline 0 & 0 \\
\hline 0 & 0 \\
\hline 0 & 0 \\
\hline 0 & 0 \\
\hline 5.263157895 & 2 \\
\hline 0.307692308 & 1 \\
\hline 0 & 0 \\
\hline 0.102092905 & 2 \\
\hline 0 & 0 \\
\hline
\end{tabular}


Basomedial amygdalar nucleus, posterior part

Subfornical organ

Substantia innominata

Subparaventricular zone

Bed nuclei of the stria terminalis

0

0

Preparasubthalamic nucleus

Parasubthalamic nucleus

Substantia nigra, compact part

$\begin{array}{rr}0 & 0 \\ 50 & 3\end{array}$

$0.333889816 \quad 2$

$0 \quad 0$

$0.641025641 \quad 1$

Supplemental somatosensory area

$100-1$

Substantia nigra, reticular part

Field CA1

Supraoptic nucleus

Medial amygdalar nucleus

Field CA2

Medial group of the dorsal thalamus

Basolateral amygdalar nucleus, ventral part

Median preoptic nucleus

Field CA3

Subthalamic nucleus

Medial mammillary nucleus

0.008221656

\section{0}

10

0.241545894

33.33333333

0.027307482

12.40310078

Subiculum

Main olfactory bulb

Medial preoptic nucleus

84.44444444

2.459016393

Medial preoptic area

1.742160279

14.66666667

Supramammillary nucleus

Cerebellar cortex

Central amygdalar nucleus

Temporal association areas

0.010026068

6.25

18.75

Tuberomammillary nucleus

15.55555556

Medial septal nucleus

0.869565217

Midline group of the dorsal thalamus

6.140350877

Nucleus of the brachium of the inferior colliculus

Triangular nucleus of septum

Claustrum

Taenia tecta

Diagonal band nucleus

Nucleus of the lateral lemniscus

Tuberal nucleus

Cuneiform nucleus

Nucleus of the lateral olfactory tract

Cortical amygdalar area

Ventral group of the dorsal thalamus

Facial motor nucleus

Visual areas

Caudoputamen

Visceral area

0.138197899 
Ventrolateral preoptic nucleus

Ventromedial hypothalamic nucleus

0
8.396946565

Orbital area

28.57142857

Dentate gyrus

Ventral tegmental area

Olfactory tubercle

Posterior amygdalar nucleus

\section{0}

0.062558649

Piriform-amygdalar area

Periaqueductal gray

Zona incerta

Dorsal peduncular area

Dorsomedial nucleus of the hypothalamus

Parabrachial nucleus

Dorsal nucleus raphe

Ectorhinal area

Entorhinal area

Posterodorsal preoptic nucleus

Perirhinal area

Posterior hypothalamic nucleus

Endopiriform nucleus, dorsal part

Epithalamus

Piriform area

Endopiriform nucleus, ventral part

Prelimbic area

Dorsal premammillary nucleus

Primary motor area

Secondary motor area

Fundus of striatum

Ventral premammillary nucleus

Geniculate group, dorsal thalamus

Geniculate group, ventral thalamus

Globus pallidus, external segment

Globus pallidus, internal segment

Peripeduncular nucleus

Gigantocellular reticular nucleus

Pedunculopontine nucleus

Gustatory areas

Presubiculum

Pontine reticular nucleus, caudal part

Pretectal region

Intercalated amygdalar nucleus

Parastrial nucleus

Prosubiculum

Ventromedial preoptic nucleus

Perifornical nucleus

Hippocampo-amygdalar transition area

Paratrochlear nucleus

$\begin{array}{rr}19.7080292 & 54 \\ 0 & 0 \\ 50 & 1 \\ 0 & 0 \\ 0 & 0 \\ 0 & 0 \\ 0 & 0 \\ 13.06818182 & 23 \\ 0 & 0 \\ 50 & 1 \\ 0.415973378 & 5 \\ 0 & 0 \\ 0 & 0 \\ 9.395973154 & 14 \\ 40 & 2 \\ 90.47619048 & 19 \\ 0 & 0 \\ 3.816793893 & 10 \\ 0 & 0 \\ 0 & 0 \\ 0 & 0 \\ 0 & 0 \\ 0 & 0 \\ 100 & 0 \\ 0 & 1 \\ 0 & 0 \\ 0 & 0 \\ 100 & 0 \\ 0 & 0 \\ 0 & 0 \\ 0 & 0 \\ 0 & 0 \\ 0 & 0 \\ 0 & 0 \\ 0 & 0 \\ 0 & 0 \\ 0 & 0 \\ 0 & 0 \\ 0 & 0 \\ 0 & 0 \\ 0 & 0 \\ 0 & 0\end{array}$


of contra cells

$$
\begin{aligned}
& \text { 능 } \\
& \text { (2) } \\
& \text { 는 } \\
& \text { (U) } \\
& \text { ก } \\
& \Xi \\
& \text { E } \\
& \text { (1) } \\
& \sum \\
& \text { (0) } \\
& \text { (2) } \\
& \text { 느 } \\
& \text { (2) } \\
& \text { (d) } \\
& \text { (U) } \\
& \text { (U) } \\
& < \\
& \text { (อ) } \\
& \text { 는 } \\
& 3 \\
& \text { (d) } \\
& \text { (1) }
\end{aligned}
$$




\begin{tabular}{|c|c|c|c|}
\hline $\begin{array}{l}\text { Periventricular } \\
\text { region }\end{array}$ & $\begin{array}{c}\text { Periventricular } \\
\text { zone }\end{array}$ & $\begin{array}{c}\text { Hypothalamic } \\
\text { medial zone }\end{array}$ & $\begin{array}{l}\text { Hypothalamic } \\
\text { lateral zone }\end{array}$ \\
\hline $\begin{array}{c}\text { Anterodorsal } \\
\text { preoptic nucleus }\end{array}$ & $\begin{array}{l}\text { Paraventricular } \\
\text { hypothalamic } \\
\text { nucleus }\end{array}$ & $\begin{array}{c}\text { Anterior } \\
\text { hypothalamic } \\
\text { nucleus }\end{array}$ & $\begin{array}{c}\text { Lateral } \\
\text { Hypothalamic area }\end{array}$ \\
\hline $\begin{array}{c}\text { Anteroventral } \\
\text { preoptic nucleus }\end{array}$ & $\begin{array}{c}\text { Periventricular } \\
\text { hypothalamic } \\
\text { nucleus, anterior }\end{array}$ & $\begin{array}{c}\text { Medial mammillary } \\
\text { nucleus }\end{array}$ & $\begin{array}{c}\text { Lateral preoptic } \\
\text { area }\end{array}$ \\
\hline $\begin{array}{l}\text { Anteroventral } \\
\text { periventricular } \\
\text { nucleus }\end{array}$ & $\begin{array}{l}\text { Periventricular } \\
\text { hypothalamic } \\
\text { nucleus, } \\
\text { intermediate }\end{array}$ & $\begin{array}{c}\text { Supramammillary } \\
\text { nucleus }\end{array}$ & $\begin{array}{c}\text { Parasubthalamic } \\
\text { nucleus }\end{array}$ \\
\hline $\begin{array}{l}\text { Dorsomedial } \\
\text { nucleus of the } \\
\text { hypothalamus }\end{array}$ & $\begin{array}{c}\text { Arcuate } \\
\text { hypothalamic } \\
\text { nucleus }\end{array}$ & $\begin{array}{c}\text { Tuberomammilary } \\
\text { nucleus }\end{array}$ & $\begin{array}{l}\text { Perifornical } \\
\text { nucleus }\end{array}$ \\
\hline $\begin{array}{c}\text { Medial preoptic } \\
\text { area }\end{array}$ & & $\begin{array}{l}\text { Medial preoptic } \\
\text { nucleus }\end{array}$ & $\begin{array}{l}\text { Retrochiasmatic } \\
\text { area }\end{array}$ \\
\hline $\begin{array}{l}\text { Posterodorsal } \\
\text { preoptic nucleus }\end{array}$ & & $\begin{array}{c}\text { Dorsal } \\
\text { premammillary } \\
\text { nucleus }\end{array}$ & $\begin{array}{l}\text { Subthalamic } \\
\text { nucleus }\end{array}$ \\
\hline Parastrial nucleus & & $\begin{array}{c}\text { Ventral } \\
\text { premammillary } \\
\text { nucleus }\end{array}$ & Tuberal nucleus \\
\hline $\begin{array}{c}\text { Periventricular } \\
\text { hypothalamic } \\
\text { nucleus, posterior }\end{array}$ & & $\begin{array}{c}\text { Ventromedial } \\
\text { hypothalamic } \\
\text { nucleus }\end{array}$ & Zona incerta \\
\hline $\begin{array}{c}\text { Periventricular } \\
\text { hypothalamic } \\
\text { nucleus, preoptic }\end{array}$ & & $\begin{array}{c}\text { Posterior } \\
\text { hypothalamic } \\
\text { nucleus }\end{array}$ & \\
\hline
\end{tabular}




\begin{tabular}{|l|l|l|l|} 
& & & \\
\hline $\begin{array}{c}\text { Ventrolateral } \\
\text { preoptic nucleus }\end{array}$ & & & \\
\hline
\end{tabular}

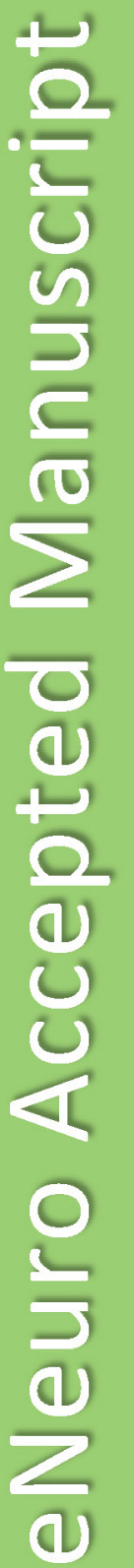




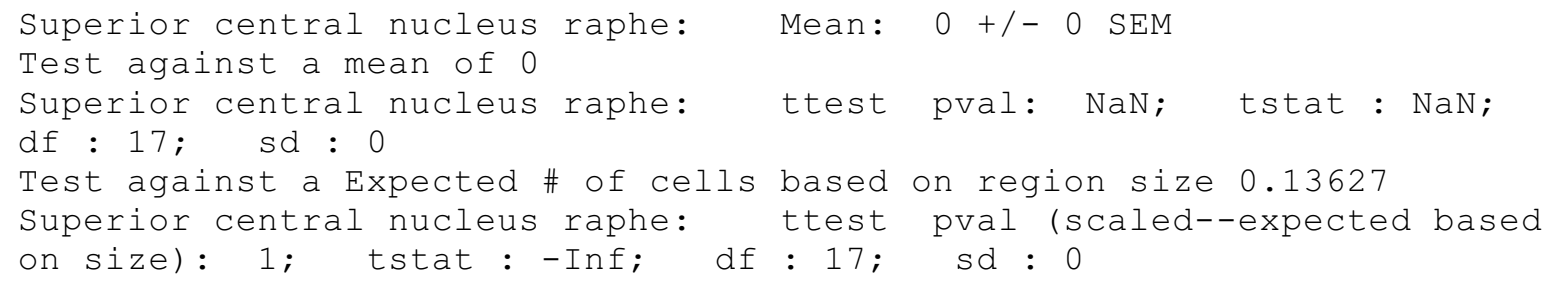

Pontine reticular nucleus: Mean: 0 +/- 0 SEM Test against a mean of 0

Pontine reticular nucleus: ttest pval: NaN; tstat : NaN; df : $\begin{array}{ll}17 ; & \text { sd : } 0 \\ \text { Test against a Expected \# of cells based on region size } 0.54093\end{array}$ Pontine reticular nucleus: ttest pval (scaled--expected based on size): 1 ; tstat : -Inf; df : 17; sd : 0

Nucleus raphe pontis: Mean: $0+/-0$ SEM

Test against a mean of 0

Nucleus raphe pontis: ttest pval: NaN; tstat : NaN; df : 17; sd : 0

Test against a Expected \# of cells based on region size 0.020984 Nucleus raphe pontis: ttest pval (scaled--expected based on size): 1; tstat : -Inf; df : 17; sd : 0

This is a confidential document and must not be discussed with others, forwarded in any form, or posted on websites without the express written consent of eNeuro. 


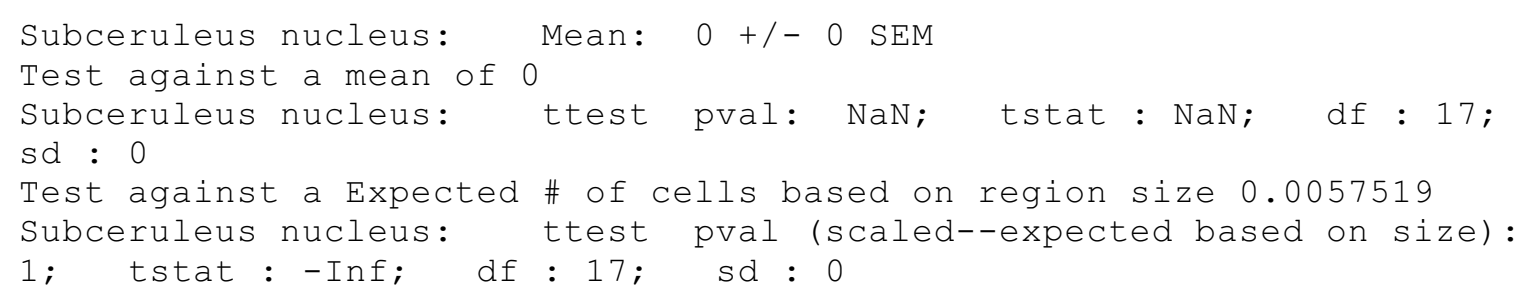

This is a confidential document and must not be discussed with others, forwarded in any form, or posted on websites without the express written consent of eNeuro. 
Test against a Expected \# of cells based on region size 0.20263

Superior olivary complex:

size): 1 ; tstat : - Inf;

ttest pral (scaled--expected based on

df : $17 ;$ sd : 0

Abducens nucleus: Mean: $0+/-0$ SEM

Test against a mean of 0

Abducens nucleus: ttest pval: NaN; tstat : NaN; df: 17; sd: 0

Test against a Expected \# of cells based on region size 0.0080405

Abducens nucleus: ttest pval (scaled--expected based on size): 1;

tstat : -Inf; df : $17 ;$ sd : 0

-------------------------

Facial motor nucleus: Mean: $0.00059285+/-0.00014795$ SEM

Test against a mean of 0

Facial motor nucleus: ttest pval: 0.16567; tstat : 1; df : 17;

sd : 0.0025152

Test against a Expected \# of cells based on region size 0.20784

Facial motor nucleus: ttest pval (scaled--expected based on size): $1 ;$ tstat : $-349.5866 ;$ df : 17; sd : 0.0025152

Accessory facial motor nucleus: Mean: 0 +/- 0 SEM

Test against a mean of 0

Accessory facial motor nucleus: ttest pval: NaN; tstat : NaN;

df : $17 ;$ sd : 0

Test against a Expected \# of cells based on region size 0.0011986

Accessory facial motor nucleus: ttest pval (scaled--expected based

on size): 1; tstat : -Inf; df : 17; sd : 0

Nucleus ambiguus: Mean: $0+/-0$ SEM

Test against a mean of 0

Nucleus ambiguus: ttest pval: NaN; tstat : NaN; df : 17; sd : 0

Test against a Expected \# of cells based on region size 0.0093817

Nucleus ambiguus: ttest pval (scaled--expected based on size): 1;

tstat : -Inf; df : 17; sd : 0

Dorsal motor nucleus of the vagus nerve: Mean: 0 +/- 0 SEM

Test against a mean of 0

Dorsal motor nucleus of the vagus nerve: ttest pval: NaN; tstat :

NaN; df : 17 ; sd : 0

Test against a Expected \# of cells based on region size 0.037822

Dorsal motor nucleus of the vagus nerve: ttest pval (scaled--

expected based on size): 1; tstat : -Inf; df : 17; sd: 0

Gigantocellular reticular nucleus: Mean: 0.003247 +/- 0.00081034 SEM Test against a mean of 0

This is a confidential document and must not be discussed with others, forwarded in any form, or posted on websites without the express written consent of eNeuro. 
Gigantocellular reticular nucleus: ttest pval: 0.16567; tstat : $1 ;$ df : 17; sd : 0.013776

Test against a Expected \# of cells based on region size 0.60393

Gigantocellular reticular nucleus: ttest pval (scaled--expected based on size): 1; tstat : $-184.9991 ;$ df : 17; sd : 0.013776

Infracerebellar nucleus: Mean: $0+/-0$ SEM

Test against a mean of 0

Infracerebellar nucleus: ttest pval: NaN; tstat : NaN; df : 17;

sd : 0

Test against a Expected \# of cells based on region size 0.011742

Infracerebellar nucleus: ttest pval (scaled--expected based on

size): 1; tstat : -Inf; df : 17; sd : 0

Inferior olivary complex: Mean: 0 +/- 0 SEM

Test against a mean of 0

Inferior olivary complex:

17 ; sd : 0

Test against a Expected \# of cells based on region size 0.11344

Inferior olivary complex: ttest pval (scaled--expected based on

size): 1; tstat : - Inf;

df : 17; sd : 0

Intermediate reticular nucleus: Mean: 0 +/- 0 SEM

Test against a mean of 0

Intermediate reticular nucleus: ttest pval: NaN; tstat : NaN;

df : 17; sd : 0

Test against a Expected \# of cells based on region size 0.60413

Intermediate reticular nucleus: ttest pval (scaled--expected based on size): 1; tstat : -Inf; df : 17; sd : 0

Inferior salivatory nucleus: Mean: 0 +/- 0 SEM

Test against a mean of 0

Inferior salivatory nucleus: ttest pval: NaN; tstat : NaN; df :

17; sd : 0

Test against a Expected \# of cells based on region size 0.0015619

Inferior salivatory nucleus: ttest pval (scaled--expected based on

size): 1; tstat : -Inf; df : 17; sd : 0

$------------------------$

Linear nucleus of the medulla: Mean: $0+/-0$ SEM

Test against a mean of 0

Linear nucleus of the medulla: ttest pval: NaN; tstat : NaN; df : 17 ; sd : 0

Test against a Expected \# of cells based on region size 0.013405

Linear nucleus of the medulla: ttest pval (scaled--expected based on size): 1; tstat : -Inf; df : 17; sd : 0

This is a confidential document and must not be discussed with others, forwarded in any form, or posted on websites without the express written consent of eNeuro. 


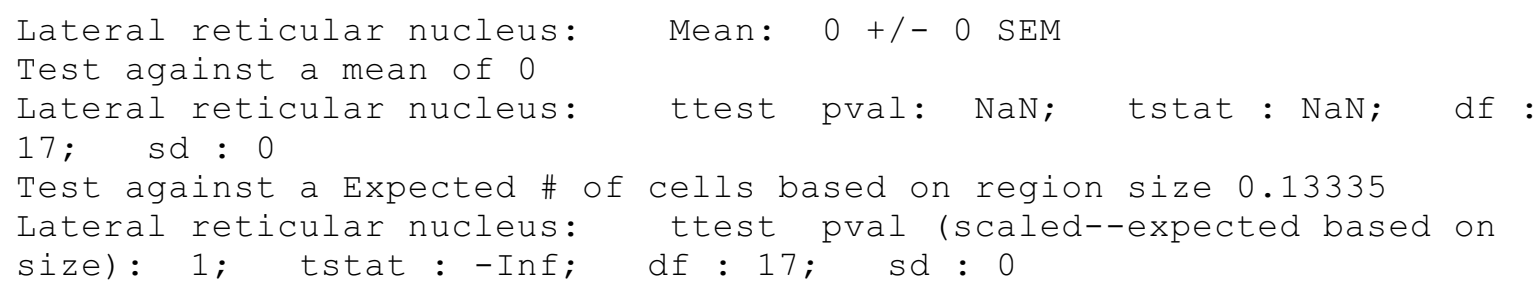

This is a confidential document and must not be discussed with others, forwarded in any form, or posted on websites without the express written consent of eNeuro. 


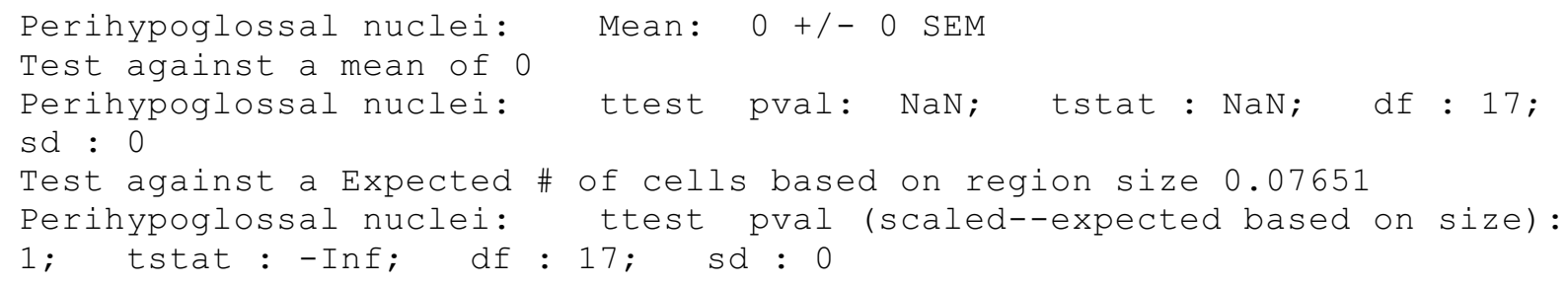

This is a confidential document and must not be discussed with others, forwarded in any form, or posted on websites without the express written consent of eNeuro. 
Nucleus raphe magnus: Mean: 0.0010423 +/-0.00018033 SEM

Test against a mean of 0

Nucleus raphe magnus: ttest pval: 0.083673; tstat : 1.4425; df : 17 ; sd : 0.0030655

Test against a Expected \# of cells based on region size 0.031972

Nucleus raphe magnus: ttest pval (scaled--expected based on size) : $1 ;$ tstat : $-42.8057 ;$ df : 17; sd : 0.0030655

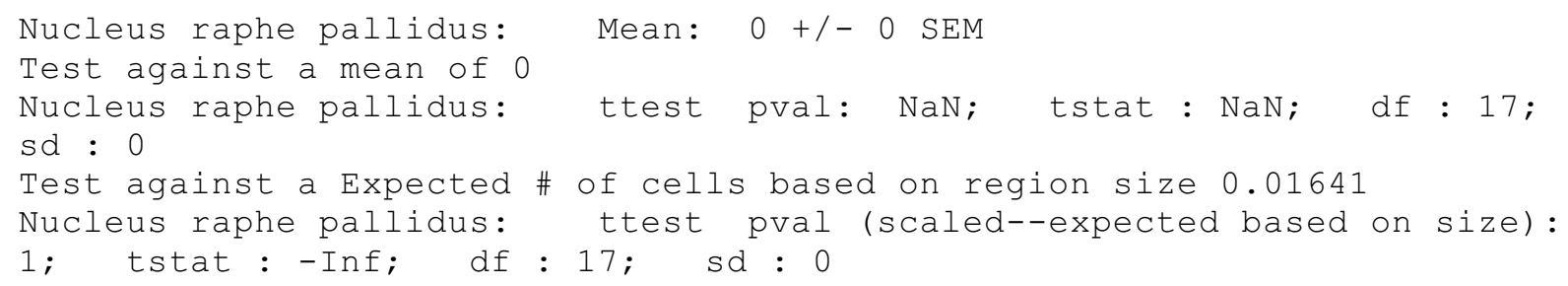

Dorsal column nuclei: Mean: $0+/-0$ SEM

Test against a mean of 0

Dorsal column nuclei: ttest pval: NaN; tstat : NaN; df : 17; sd : 0

Test against a Expected \# of cells based on region size 0.09435

Dorsal column nuclei: ttest pval (scaled--expected based on size):

This is a confidential document and must not be discussed with others, forwarded in any form, or posted on websites without the express written consent of eNeuro. 
1; tstat : -Inf; df : $17 ;$ sd : 0

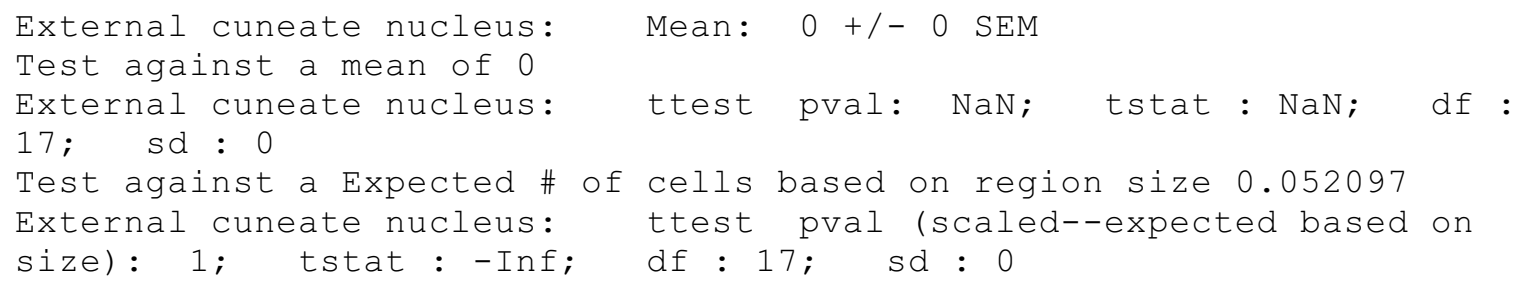

Nucleus of the trapezoid body: Mean: 0 +/- 0 SEM

Test against a mean of 0

Nucleus of the trapezoid body: ttest pval: NaN; tstat : NaN; df : 17 ; sd : 0

Test against a Expected \# of cells based on region size 0.0397

Nucleus of the trapezoid body: ttest pval (scaled--expected based on size): 1; tstat : -Inf; df : 17; sd : 0

Nucleus of the solitary tract: Mean: $0+/-0$ SEM

Test against a mean of 0

Nucleus of the solitary tract: ttest pval: NaN; tstat : NaN; df : 17 ; sd : 0

Test against a Expected \# of cells based on region size 0.19355

Nucleus of the solitary tract: ttest pval (scaled--expected based on size): 1 ; tstat : -Inf; df : 17; sd : 0

Spinal nucleus of the trigeminal, caudal part: Mean: 0 +/- 0 SEM Test against a mean of 0

Spinal nucleus of the trigeminal, caudal part: ttest pval: NaN; tstat : NaN; df : $17 ;$ sd : 0

Test against a Expected \# of cells based on region size 0.3686

Spinal nucleus of the trigeminal, caudal part: ttest pval (scaled-expected based on size): 1; tstat : -Inf; df : 17; sd: 0

Spinal nucleus of the trigeminal, interpolar part: $\quad$ Mean: $0+/-0$ SEM Test against a mean of 0

Spinal nucleus of the trigeminal, interpolar part: ttest pval: NaN; tstat : NaN; df : 17 ; sd : 0

Test against a Expected \# of cells based on region size 0.4143

Spinal nucleus of the trigeminal, interpolar part: ttest pval (scaled--expected based on size): 1; tstat : -Inf; df : 17; sd: 0

Spinal nucleus of the trigeminal, oral part: Mean: 0 +/- 0 SEM Test against a mean of 0

Spinal nucleus of the trigeminal, oral part: ttest pval: NaN;

This is a confidential document and must not be discussed with others, forwarded in any form, or posted on websites without the express written consent of eNeuro. 
tstat : NaN; df: $17 ;$ sd : 0

Test against a Expected \# of cells based on region size 0.21959

Spinal nucleus of the trigeminal, oral part: ttest pval (scaled--

expected based on size): 1; tstat : -Inf; df : 17; sd : 0

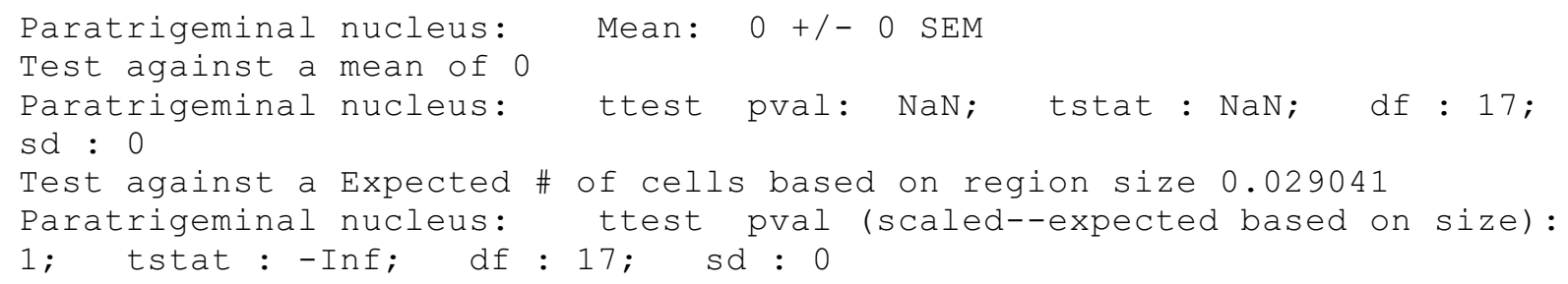

Barrington's nucleus: Mean: 0 +/- 0 SEM

Test against a mean of 0

Barrington's nucleus: ttest pval: NaN; tstat : NaN; df: 17; sd : 0

Test against a Expected \# of cells based on region size 0.0033751

Barrington's nucleus: ttest pval (scaled--expected based on size) : $1 ;$ tstat : -Inf; df : 17; sd : 0

$------------------------$

Dorsal tegmental nucleus: Mean: $0+/-0$ SEM

Test against a mean of 0

Dorsal tegmental nucleus: ttest pval: NaN; tstat : NaN; df :

17 isd : 0

Test against a Expected \# of cells based on region size 0.022536

Dorsal tegmental nucleus:

size): 1 ; tstat : -Inf;

ttest pval (scaled--expected based on

df : $17 ;$ sd : 0

Posterodorsal tegmental nucleus: Mean: $0+1-0$ SEM

Test against a mean of 0

Posterodorsal tegmental nucleus: ttest pval: NaN; tstat : NaN;

df : $17 ;$ sd : 0

Test against a Expected \# of cells based on region size 0.010397

Posterodorsal tegmental nucleus: test pval (scaled--expected based on size): 1; tstat : -Inf; df : 17; sd : 0

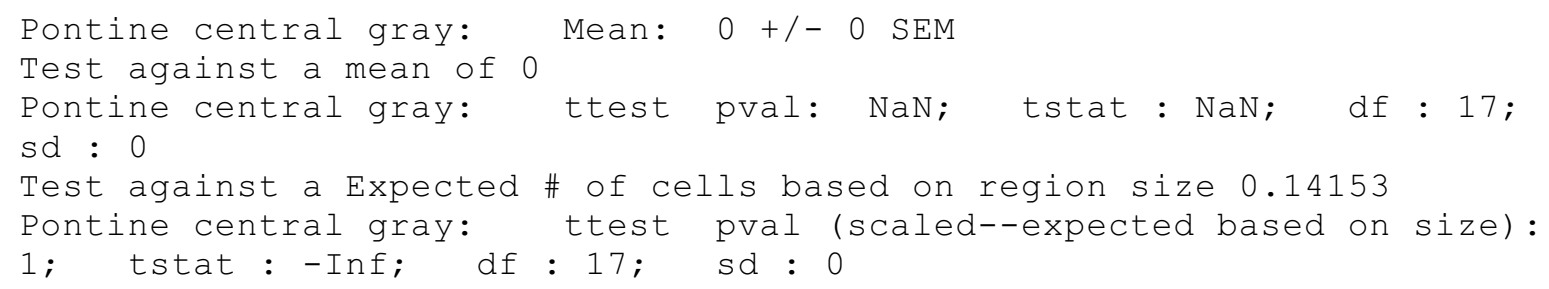

Pontine gray: Mean: $0+/-0$ SEM

This is a confidential document and must not be discussed with others, forwarded in any form, or posted on websites without the express written consent of eNeuro. 
Test against a mean of 0

Pontine gray: ttest pval: NaN; tstat : NaN; df : 17; sd : 0

Test against a Expected \# of cells based on region size 0.21287

Pontine gray: ttest pval (scaled--expected based on size): 1;

tstat : -Inf; df : $17 ;$ sd : 0

Pontine reticular nucleus, caudal part: Mean: 0.003247 +/-

0.00081034 SEM

Test against a mean of 0

Pontine reticular nucleus, caudal part: ttest pval: 0.16567;

tstat : 1; df : 17; sd : 0.013776

Test against a Expected \# of cells based on region size 0.54369

Pontine reticular nucleus, caudal part: ttest pval (scaled--expected

based on size): 1; tstat : -166.4446 ; df : 17; sd : 0.013776

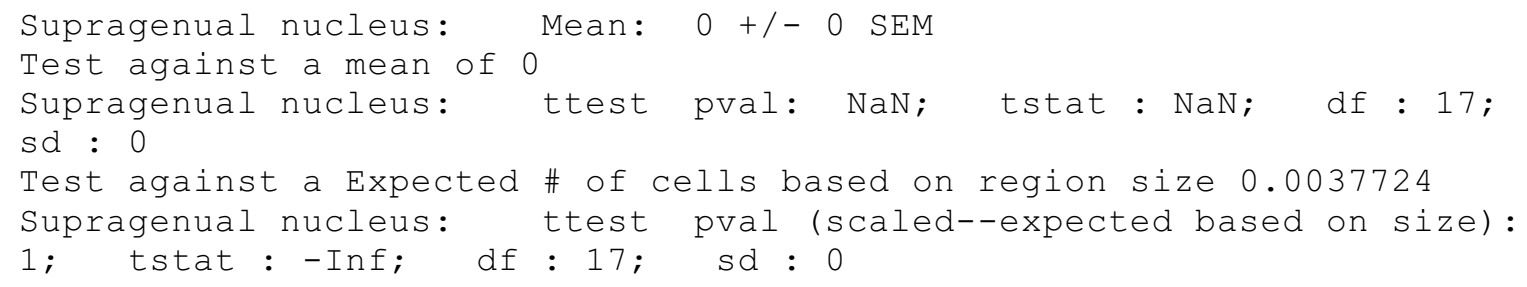

Supratrigeminal nucleus:

Test against a mean of 0

Supratrigeminal nucleus:

sd : 0

Test against a Expected \# of cells based on region size 0.056501

Supratrigeminal nucleus: ttest pval (scaled--expected based on

size): 1; tstat : -Inf; df : 17; sd : 0

Tegmental reticular nucleus:

Test against a mean of 0

Tegmental reticular nucleus:

17; sd : 0

Test against a Expected \# of

Tegmental reticular nucleus:

size): 1 ; tstat : -Inf;

size):--------------------

Motor nucleus of trigeminal:

Test against a mean of 0

Motor nucleus of trigeminal:

17; sd : 0

Test against a Expected \# o

Motor nucleus of trigeminal:

size): 1 ; tstat : - Inf;

size): 1, tstat:

Mean: $0+/-0$ SEM

test pval: NaN; tstat : NaN; df : 17;

Mean: $0+/-0$ SEM

test pval: NaN; tstat : NaN; df :

cells based on region size 0.18094

ttest pval (scaled--expected based on df : $17 ;$ sd : 0

This is a confidential document and must not be discussed with others, forwarded in any form, or posted on websites without the express written consent of eNeuro. 


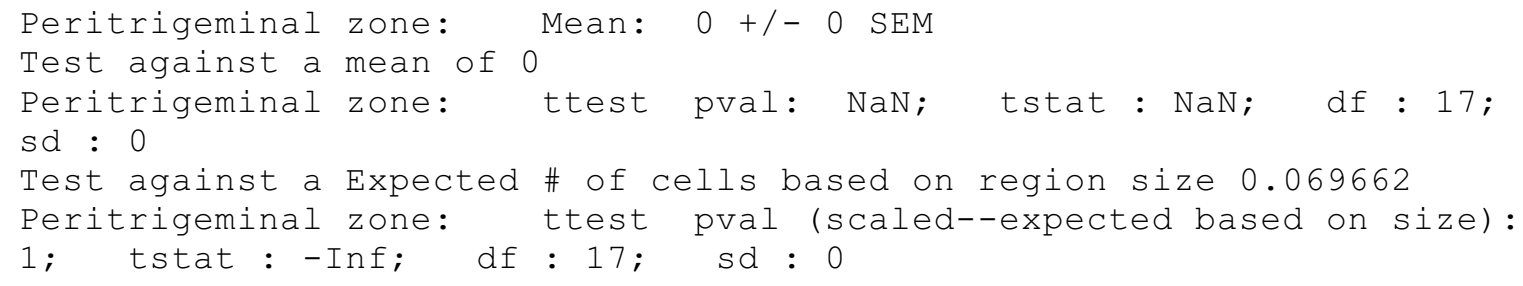

Accessory trigeminal nucleus: Mean: $0+/-0$ SEM

Test against a mean of 0

Accessory trigeminal nucleus: ttest pval: NaN; tstat : NaN; df : 17; sd : 0

Test against a Expected \# of cells based on region size 0.0031035

Accessory trigeminal nucleus: ttest pval (scaled--expected based on size): 1; tstat : -Inf; df : 17; sd : 0

Parvicellular motor 5 nucleus: Mean: 0 +/- 0 SEM

Test against a mean of 0

Parvicellular motor 5 nucleus: ttest pval: NaN; tstat : NaN; df : 17 ; sd : 0

Test against a Expected \# of cells based on region size 0.018285

Parvicellular motor 5 nucleus: ttest pval (scaled--expected based on size): 1 ; tstat : -Inf; df : 17; sd : 0

Intertrigeminal nucleus: Test against a mean of 0 Intertrigeminal nucleus: sd : 0

Test against a Expected \# of cells based on region size 0.0095718

Intertrigeminal nucleus: ttest pval (scaled--expected based on size): 1; tstat : -Inf;
Mean: $0+/-0$ SEM

test pval: NaN; tstat : NaN; df : 17;

Field CA1: Mean: $2.4885+/-0.18631$ SEM

Test against a mean of 0

Field CA1: ttest pval: 0.0019677 ; tstat : 3.3334; df: 17; sd : 3.1672

Test against a Expected \# of cells based on region size 2.6586

Field CA1: ttest pval (scaled--expected based on size): 0.58877;

tstat : -0.22787 ; df : 17; sd : 3.1672

Field CA2: Mean: $0.25768+/-0.023006$ SEM

Test against a mean of 0

Field CA2: ttest pval: 0.0062132 ; tstat : 2.7953; df: 17; sd : 0.3911

Test against a Expected \# of cells based on region size 0.12892

Field CA2: ttest pval (scaled--expected based on size): 0.090217;

This is a confidential document and must not be discussed with others, forwarded in any form, or posted on websites without the express written consent of eNeuro. 
tstat : 1.3968; df : $17 ;$ sd : 0.3911

FieldCa3: Mean: 6.3221 +/-0.57746 SEM

Test against a mean of 0

FieldCa3: ttest pval: 0.0070927 ; tstat : 2.7323; df : 17; sd : 9.8169

Test against a Expected \# of cells based on region size 1.8646

FieldCa3: ttest pval (scaled--expected based on size): 0.035471;

tstat : 1.9264; df : 17; sd : 9.8169

Dentate Gyrus: Mean: 0.018308 +/- 0.0028243 SEM

Test against a mean of 0

Dentate Gyrus: ttest pval: 0.06206 ; tstat : 1.6177; df: 17;

sd : 0.048013

Test against a Expected \# of cells based on region size 1.5904

Dentate Gyrus: ttest pval (scaled--expected based on size): 1;

tstat : $-138.9174 ;$ df : 17; sd : 0.048013

Fasciola cinerea: Mean: $0+/-0$ SEM

Test against a mean of 0

Fasciola cinerea: ttest pval: NaN; tstat : NaN; df : 17; sd : 0

Test against a Expected \# of cells based on region size 0.051896

Fasciola cinerea: ttest pval (scaled--expected based on size): 1;

tstat : -Inf; df : 17; sd : 0

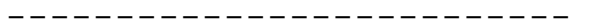

Induseum griseum: Mean: $0+/-0$ SEM

Test against a mean of 0

Induseum griseum: ttest pval: NaN; tstat : NaN; df : 17; sd : 0

Test against a Expected \# of cells based on region size 0.052127

Induseum griseum: ttest pval (scaled--expected based on size): 1; tstat : -Inf; df : 17; sd : 0

$--------------------------$

Entorhinal area: Mean: $0.25717+/-0.026799$ SEM

Test against a mean of 0

Entorhinal area: ttest pval: 0.014209; tstat : 2.3949; df : 17; sd : 0.45559

Test against a Expected \# of cells based on region size 2.4996

Entorhinal area: ttest pval (scaled--expected based on size): 1;

tstat : $-20.8826 ;$ df : 17; sd : 0.45559

Parasubiculum: Mean: $0+/-0$ SEM

Test against a mean of 0

Parasubiculum: ttest pval: NaN; tstat : NaN; df : 17; sd : 0 Test against a Expected \# of cells based on region size 0.20365

This is a confidential document and must not be discussed with others, forwarded in any form, or posted on websites without the express written consent of eNeuro. 


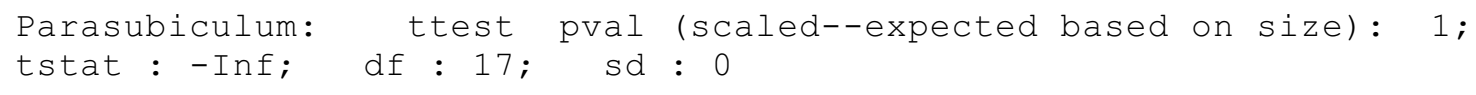

This is a confidential document and must not be discussed with others, forwarded in any form, or posted on websites without the express written consent of eNeuro. 
Test against a Expected \# of cells based on region size 0.071492

Area prostriata: ttest pval (scaled--expected based on size): 1;

tstat : -Inf; df: $17 ;$ sd : 0

Median eminence: $\quad$ Mean: $0+/-0$ SEM

Test against a mean of 0

Median eminence: ttest pval: NaN; tstat : NaN; df : 17; sd :

0

Test against a Expected \# of cells based on region size 0.018196

Median eminence: ttest pval (scaled--expected based on size): 1;

tstat : -Inf; df : 17 ; sd : 0

Anterodorsal preoptic nucleus: Mean: 0.015989 +/-0.0016003 SEM

Test against a mean of 0

Anterodorsal preoptic nucleus: ttest pval: 0.01163 ; tstat :

2.4934; df : 17; sd : 0.027206

Test against a Expected \# of cells based on region size 0.021989

Anterodorsal preoptic nucleus: ttest pval (scaled--expected based on size): 0.81873 ; tstat : $-0.93569 ;$ df : 17; sd : 0.027206

Anteroventral preoptic nucleus: Mean: $0.070683+/-0.0059333$ SEM

Test against a mean of 0

Anteroventral preoptic nucleus: ttest pval: 0.0042651 ; tstat :

2.9731; df : 17; sd : 0.10087

Test against a Expected \# of cells based on region size 0.02003

Anteroventral preoptic nucleus: ttest pval (scaled--expected based on size): 0.024012 ; tstat : 2.1306 ; df : 17; sd : 0.10087

Anteroventral periventricular nucleus: $\quad$ Mean: $0.051614+/-0.0055466$

SEM

Test against a mean of 0

Anteroventral periventricular nucleus: test pval: 0.016441;

tstat : 2.3224; df: $17 ;$ sd : 0.094292

Test against a Expected \# of cells based on region size 0.070762

Anteroventral periventricular nucleus: ttest pval (scaled--expected

based on size): 0.79953; tstat : -0.86153 ; df : 17; sd : 0.094292

Dorsomedial nucleus of the hypothalamus: Mean: $0.4397+/-0.047233$

SEM

Test against a mean of 0

Dorsomedial nucleus of the hypothalamus: ttest pval: 0.016412;

tstat : 2.3233; df: $17 ;$ sd : 0.80295

Test against a Expected \# of cells based on region size 0.081722

Dorsomedial nucleus of the hypothalamus: ttest pval (scaled--

expected based on size): 0.037863; tstat : 1.8915; df: 17; sd: 0.80295

This is a confidential document and must not be discussed with others, forwarded in any form, or posted on websites without the express written consent of eNeuro. 
Median preoptic nucleus: Test against a mean of 0 Median preoptic nucleus: df : 17; sd : 0.0055635 Test against a Expected \# of cells based on region size 0.01748 Median preoptic nucleus: ttest pval (scaled--expected based on size): 1; tstat : $-11.8748 ;$ df : 17; sd : 0.0055635

Medial preoptic area: Mean: $0.25786+/-0.019784$ SEM

Test against a mean of 0

Medial preoptic area: ttest pval: 0.0023415 ; tstat : 3.2528; df : 17 ; sd : 0.33633

Test against a Expected \# of cells based on region size 0.12125 Medial preoptic area: ttest pval (scaled--expected based on size): 0.051482 ; tstat : $1.7233 ;$ df $: 17 ;$ sd : 0.33633

Vascular organ of the lamina terminalis: Mean: 0 +/- 0 SEM Test against a mean of 0

Vascular organ of the lamina terminalis: ttest pval: NaN; tstat : NaN; df : 17 ; sd : 0

Test against a Expected \# of cells based on region size 0.0030865

Vascular organ of the lamina terminalis: ttest pval (scaled-expected based on size): 1; tstat : -Inf; df : 17 ; sd: 0

Posterodorsal preoptic nucleus: Mean: $0.0086461+/-0.00093346$ SEM Test against a mean of 0

Posterodorsal preoptic nucleus: ttest pval: 0.0168 ; tstat : 2.3116; df: 17 ; sd : 0.015869

Test against a Expected \# of cells based on region size 0.0025228

Posterodorsal preoptic nucleus: ttest pval (scaled--expected based on size): 0.059994 ; tstat : 1.6371; df : 17; sd : 0.015869

Parastrial nucleus: Mean: $0.012037+/-0.0015809$ SEM

Test against a mean of 0

Parastrial nucleus: ttest pval: 0.037248; tstat : 1.9003; df :

17 i sd : 0.026875

Test against a Expected \# of cells based on region size 0.02096

Parastrial nucleus: ttest pval (scaled--expected based on size):

$0.91151 ;$ tstat : $-1.4086 ;$ df : 17; sd : 0.026875

--------------------------

Periventricular hypothalamic nucleus, posterior part: $\quad$ Mean: 0.069225 $+/-0.0081592$ SEM

Test against a mean of 0

Periventricular hypothalamic nucleus, posterior part: ttest pval:

$0.024635 ;$ tstat : 2.1174; df : 17; sd : 0.13871

Test against a Expected \# of cells based on region size 0.031252

This is a confidential document and must not be discussed with others, forwarded in any form, or posted on websites without the express written consent of eNeuro. 
Periventricular hypothalamic nucleus, posterior part: ttest pval (scaled--expected based on size): 0.13074; tstat : 1.1615; df : 17; sd : 0.13871

Periventricular hypothalamic nucleus, preoptic part: Mean: 0.035883 $+/-0.0042756$ SEM

Test against a mean of 0

Periventricular hypothalamic nucleus, preoptic part: ttest pval:

0.025751 ; tstat : 2.0945; df : 17; sd : 0.072685

Test against a Expected \# of cells based on region size 0.045852

Periventricular hypothalamic nucleus, preoptic part: ttest pval

(scaled--expected based on size): 0.71587; tstat : -0.5819 ; df :

17; sd : 0.072685

\begin{abstract}
Subparaventricular zone: Mean: $0.0041656+/-0.00060095$ SEM
Test against a mean of 0

Subparaventricular zone:

df : 17; sd : 0.010216

Test against a Expected \# of cells based on region size 0.023795

Subparaventricular zone: ttest pval (scaled--expected based on

size): 1; tstat : $-8.152 ;$ df : 17; sd : 0.010216
\end{abstract}

--------------------------

Suprachiasmatic nucleus:

Test against a mean of 0

Suprachiasmatic nucleus:

df : 17; sd : 0.017853

Test against a Expected \# of cells based on region size 0.02074

Suprachiasmatic nucleus: ttest pval (scaled--expected based on

size): 0.99932; tstat : $-3.8213 ;$ df : 17; sd: 0.017853

Subfornical organ: Mean: 0.0011857 +/-0.00029591 SEM

Test against a mean of 0

Subfornical organ: ttest pval: 0.16567 ; tstat : 1; df: 17;

sd : 0.0050305

Test against a Expected \# of cells based on region size 0.025211

Subfornical organ: ttest pval (scaled--expected based on size): 1;

tstat : $-20.263 ;$ df $: 17 ;$ sd : 0.0050305

------------------------

Ventromedial preoptic nucleus: Mean: $0.0059082+/-0.0010758$ SEM

Test against a mean of 0

Ventromedial preoptic nucleus: ttest pval: 0.094157; tstat :

$1.3706 ;$ df : 17; sd : 0.018288

Test against a Expected \# of cells based on region size 0.023541

Ventromedial preoptic nucleus: ttest pval (scaled--expected based on size): 0.99962 ; tstat : $-4.0906 ;$ df : 17; sd : 0.018288

This is a confidential document and must not be discussed with others, forwarded in any form, or posted on websites without the express written consent of eNeuro. 


\begin{abstract}
Ventrolateral preoptic nucleus: Mean: $0.012442+/-0.0026021$ SEM
Test against a mean of 0

Ventrolateral preoptic nucleus: ttest pval: 0.12458 ; tstat :

1.1933; df : 17; sd : 0.044236

Test against a Expected \# of cells based on region size 0.02605

Ventrolateral preoptic nucleus: ttest pval (scaled--expected based

on size): 0.89539 ; tstat : $-1.3052 ;$ df : 17; sd : 0.044236
\end{abstract}

Supraoptic nucleus: Mean: $0.0081396+/-0.0014333$ SEM

Test against a mean of 0

Supraoptic nucleus: ttest pval: 0.087242 ; tstat : 1.4172; df :

17; sd : 0.024367

Test against a Expected \# of cells based on region size 0.030766

Supraoptic nucleus: ttest pval (scaled--expected based on size):

0.99947 ; tstat : $-3.9397 ;$ df : 17; sd : 0.024367

Accessory supraoptic group: Mean: 0.0012484 +/- 0.00031157 SEM

Test against a mean of 0

Accessory supraoptic group: ttest pval: 0.16567; tstat: 1; df

: 17 ; sd : 0.0052967

Test against a Expected \# of cells based on region size 0.0013174

Accessory supraoptic group: ttest pval (scaled--expected based on

size): 0.52172 ; tstat : -0.055272 ; df : 17; sd : 0.0052967

Paraventricular hypothalamic nucleus: $\quad$ Mean: $0.03159+/-0.0059345$

SEM

Test against a mean of 0

Paraventricular hypothalamic nucleus: ttest pval: 0.10079; tstat : 1.3285; df : 17; sd : 0.10089

Test against a Expected \# of cells based on region size 0.044498

Paraventricular hypothalamic nucleus: ttest pval (scaled--expected

based on size): 0.70285 ; tstat : -0.54283 ; df : 17; sd: 0.10089

Periventricular hypothalamic nucleus, anterior part: $\quad$ Mean: 0.0051395 $+/-0.00090238$ SEM

Test against a mean of 0

Periventricular hypothalamic nucleus, anterior part: test pval:

$0.086644 ;$ tstat : 1.4214; df : 17; sd : 0.015341

Test against a Expected \# of cells based on region size 0.014353

Periventricular hypothalamic nucleus, anterior part: ttest pval

(scaled--expected based on size): 0.9896; tstat : -2.548 ; df: 17;

sd : 0.015341

Periventricular hypothalamic nucleus, intermediate part: Mean: $0.082574+/-0.0049426$ SEM

Test against a mean of 0

Periventricular hypothalamic nucleus, intermediate part: ttest pval:

This is a confidential document and must not be discussed with others, forwarded in any form, or posted on websites without the express written consent of eNeuro. 
$0.00032136 ; \quad$ tstat : 4.1694; df : 17; sd : 0.084025

Test against a Expected \# of cells based on region size 0.056755

Periventricular hypothalamic nucleus, intermediate part: ttest pval

(scaled--expected based on size): 0.10487; tstat : 1.3037; df : 17; sd : 0.084025

Arcuate hypothalamic nucleus: Mean: 0.12561 +/- 0.023991 SEM

Test against a mean of 0

Arcuate hypothalamic nucleus: ttest pval: 0.10435 ; tstat :

1.3067; df : 17; sd : 0.40784

Test against a Expected \# of cells based on region size 0.066881

Arcuate hypothalamic nucleus: ttest pval (scaled--expected based on size): 0.27464 ; tstat : 0.61099 ; df : 17; sd : 0.40784

Lateral hypothalamic area: Mean: $2.007+/-0.16859$ SEM

Test against a mean of 0

Lateral hypothalamic area: ttest pval: 0.0042832; tstat : 2.9711;

df : $17 ;$ sd : 2.866

Test against a Expected \# of cells based on region size 0.47743

Lateral hypothalamic area: ttest pval (scaled--expected based on size): 0.018459 ; tstat : 2.2643 ; df : 17; sd : 2.866

--------------------------

Lateral preoptic area:

Test against a mean of 0

Lateral preoptic area:

df : 17 ; sd : 0.37562

Test against a Expected \# of cells based on region size 0.12063

Lateral preoptic area: ttest pval (scaled--expected based on size) : $0.0081346 ;$ tstat $: 2.6666 ;$ df $: 17 ;$ sd : 0.37562

$--------------------------$

Preparasubthalamic nucleus: $\quad$ Mean: 0.017012 +/- 0.0018394 SEM

Test against a mean of 0

Preparasubthalamic nucleus: ttest pval: 0.016913; tstat : 2.3082;

df : 17; sd : 0.031269

Test against a Expected \# of cells based on region size 0.0039048

Preparasubthalamic nucleus: ttest pval (scaled--expected based on

size): 0.04661 ; tstat : 1.7784; df : 17; sd : 0.031269

Parasubthalamic nucleus: Mean: $0.29958+/-0.034813$ SEM

Test against a mean of 0

Parasubthalamic nucleus:

test pval: 0.023226 ; tstat : 2.1477;

df : 17; sd : 0.59182

Test against a Expected \# of cells based on region size 0.041815

Parasubthalamic nucleus: ttest pval (scaled--expected based on size): 0.041046 ; tstat : $1.8479 ;$ df : 17; sd : 0.59182

This is a confidential document and must not be discussed with others, forwarded in any form, or posted on websites without the express written consent of eNeuro. 


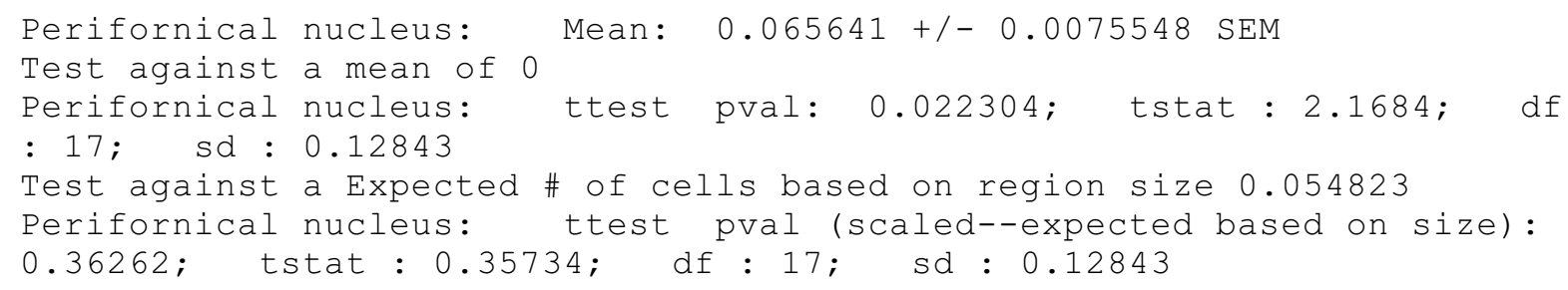

This is a confidential document and must not be discussed with others, forwarded in any form, or posted on websites without the express written consent of eNeuro. 
Lateral mammilary nucleus: Mean: 0.011553 +/- 0.0016939 SEM Test against a mean of 0 Lateral mammillary nucleus: ttest pval: 0.053473; tstat : 1.7021; df : 17; sd : 0.028796

Test against a Expected \# of cells based on region size 0.020234

Lateral mammillary nucleus: ttest pval (scaled--expected based on size): 0.89096 ; tstat : $-1.279 ;$ df : 17; sd : 0.028796

Medial mammillary nucleus: Mean: $0.39412+/-0.050426$ SEM Test against a mean of 0 Medial mammillary nucleus: ttest pval: 0.033895; tstat : 1.9506; df : 17; sd : 0.85724 Test against a Expected \# of cells based on region size 0.13169 Medial mammillary nucleus: ttest pval (scaled--expected based on size): 0.10567 ; tstat : 1.2988; df : 17; sd : 0.85724

Supramammillary nucleus: Test against a mean of 0 Supramammillary nucleus: df : 17 ; sd : 0.18529 Test against a Expected \# of cells based on region size 0.066235 Supramammillary nucleus: ttest pval (scaled--expected based on size): 0.42733 ; tstat : $0.18597 ;$ df : 17; sd: 0.18529

Tuberomammillary nucleus: $\quad$ Mean: 0.081479 +/ 0.010209 SEM Test against a mean of 0 Tuberomammillary nucleus: df : 17; sd : 0.17355 Test against a Expected \# of cells based on region size 0.040192 Tuberomammillary nucleus: ttest pval (scaled--expected based on size): 0.16348 ; tstat : 1.0093; df : 17; sd : 0.17355

Medial preoptic nucleus: Test against a mean of 0 Medial preoptic nucleus: df : 17; sd : 0.55775 Test against a Expected \# Medial preoptic nucleus: size): 0.089916 ; tstat

\author{
Mean: $0.27183+/-0.032809$ SEM \\ test pval: 0.027118 ; tstat : 2.0677;
}

Dorsal premammillary nucleus: Mean: $0.23528+/-0.032657$ SEM Test against a mean of 0

Dorsal premammillary nucleus: ttest pval: 0.044981; tstat : 1.798; df : 17; sd : 0.55517 Test against a Expected \# of cells based on region size 0.028634

This is a confidential document and must not be discussed with others, forwarded in any form, or posted on websites without the express written consent of eNeuro. 
Dorsal premammillary nucleus: ttest pval (scaled--expected based on size): 0.066362; tstat : 1.5792; df : 17; sd : 0.55517

Ventral premammillary nucleus: Mean: $0.49484+/-0.11132$ SEM Test against a mean of 0

Ventral premammillary nucleus: ttest pval: 0.14135; tstat : $1.1094 ;$ df : 17; sd : 1.8924

Test against a Expected \# of cells based on region size 0.043666

Ventral premammillary nucleus: ttest pval (scaled--expected based on size): 0.16298; tstat : 1.0115; df : 17; sd : 1.8924

Paraventricular hypothalamic nucleus, descending division: Mean: $0.013922+/-0.0012852$ SEM

Test against a mean of 0

Paraventricular hypothalamic nucleus, descending division: ttest pval: 0.0075325; tstat : 2.7035; df : 17; sd : 0.021848

Test against a Expected \# of cells based on region size 0.028634

Paraventricular hypothalamic nucleus, descending division: ttest pval (scaled--expected based on size): 0.99454; tstat : -2.8569 ; : 17 ; sd : 0.021848

Ventromedial hypothalamic nucleus: Mean: $1.2151+/-0.16121$ SEM Test against a mean of 0

Ventromedial hypothalamic nucleus: ttest pval: 0.038606 ; tstat : 1.881; df : 17; sd : 2.7406

Test against a Expected \# of cells based on region size 0.11942

Ventromedial hypothalamic nucleus: ttest pval (scaled--expected based on size): 0.054046; tstat : 1.6962; df : 17; sd : 2.7406

Posterior hypothalamic nucleus: Mean: $0.13119+/-0.011118$ SEM Test against a mean of 0

Posterior hypothalamic nucleus: ttest pval: 0.0045287; tstat : 2.9449; df : 17; sd : 0.189

Test against a Expected \# of cells based on region size 0.15853

Posterior hypothalamic nucleus: ttest pval (scaled--expected based on size): 0.72627 ; tstat : -0.61383 ; df : 17; sd : 0.189

Substantia nigra, reticular part: Mean: 0.037884 +/-0.0052302 SEM Test against a mean of 0

Substantia nigra, reticular part: ttest pval: 0.044192; tstat : 1.8077; df : 17; sd : 0.088913 Test against a Expected \# of cells based on region size 0.43287 Substantia nigra, reticular part: ttest pval (scaled--expected based on size): 1; tstat : -18.8472 ; df : 17; sd : 0.088913

--------------------------

Ventral tegmental area: Mean: 0.045944 +/- 0.0064395 SEM

This is a confidential document and must not be discussed with others, forwarded in any form, or posted on websites without the express written consent of eNeuro. 
Test against a mean of 0

Ventral tegmental area: df : 17; sd : 0.10947

Test against a Expected \# of cells based on region size 0.11082

Ventral tegmental area: ttest pval (scaled--expected based on size): 0.98886 ; tstat : $-2.5143 ;$ df : 17; sd : 0.10947

Paranigral nucleus: Mean: $0+/-0$ SEM

Test against a mean of 0

Paranigral nucleus: ttest pval: NaN; tstat : NaN; df : 17; sd : 0

Test against a Expected \# of cells based on region size 0.004774

Paranigral nucleus: ttest pval (scaled--expected based on size): 1; tstat : -Inf; df : 17; sd : 0

Midbrain reticular nucleus, retrorubral area: Mean: 0.0012484 +/0.00031157 SEM

Test against a mean of 0

Midbrain reticular nucleus, retrorubral area: ttest pval: 0.16567;

tstat : 1; df : 17; sd : 0.0052967

Test against a Expected \# of cells based on region size 0.030233

Midbrain reticular nucleus, retrorubral area: ttest pval (scaled--

expected based on size): 1; tstat : -23.2169 ; df : 17; sd :

0.0052967

Midbrain reticular nucleus: Mean: $0.10224+/-0.018492$ SEM

Test against a mean of 0

Midbrain reticular nucleus: ttest pval: 0.092747; tstat : 1.3799;

df : 17; sd : 0.31436

Test against a Expected \# of cells based on region size 1.1584

Midbrain reticular nucleus: ttest pval (scaled--expected based on

size): 1; tstat : $-14.2545 ;$ df : 17; sd : 0.31436

Superior colliculus, motor related: Mean: 0.13275 +/- 0.022439 SEM

Test against a mean of 0

Superior colliculus, motor related: ttest pval: 0.079047; tstat :

1.4765; df : 17; sd : 0.38146

Test against a Expected \# of cells based on region size 1.2407

Superior colliculus, motor related: ttest pval (scaled--expected

based on size): 1; tstat : -12.3225 ; df : 17; sd : 0.38146

Periaqueductal gray: Mean: 0.0050446 +/- 0.00090285 SEM

Test against a mean of 0

Periaqueductal gray: ttest pval: 0.090573; tstat : 1.3944 ; df :

17; sd : 0.015348

Test against a Expected \# of cells based on region size 1.0825

Periaqueductal gray: ttest pval (scaled--expected based on size):

This is a confidential document and must not be discussed with others, forwarded in any form, or posted on websites without the express written consent of eNeuro. 
$1 ; \quad$ tstat : $-297.8342 ;$ df $: 17 ;$ sd : 0.015348

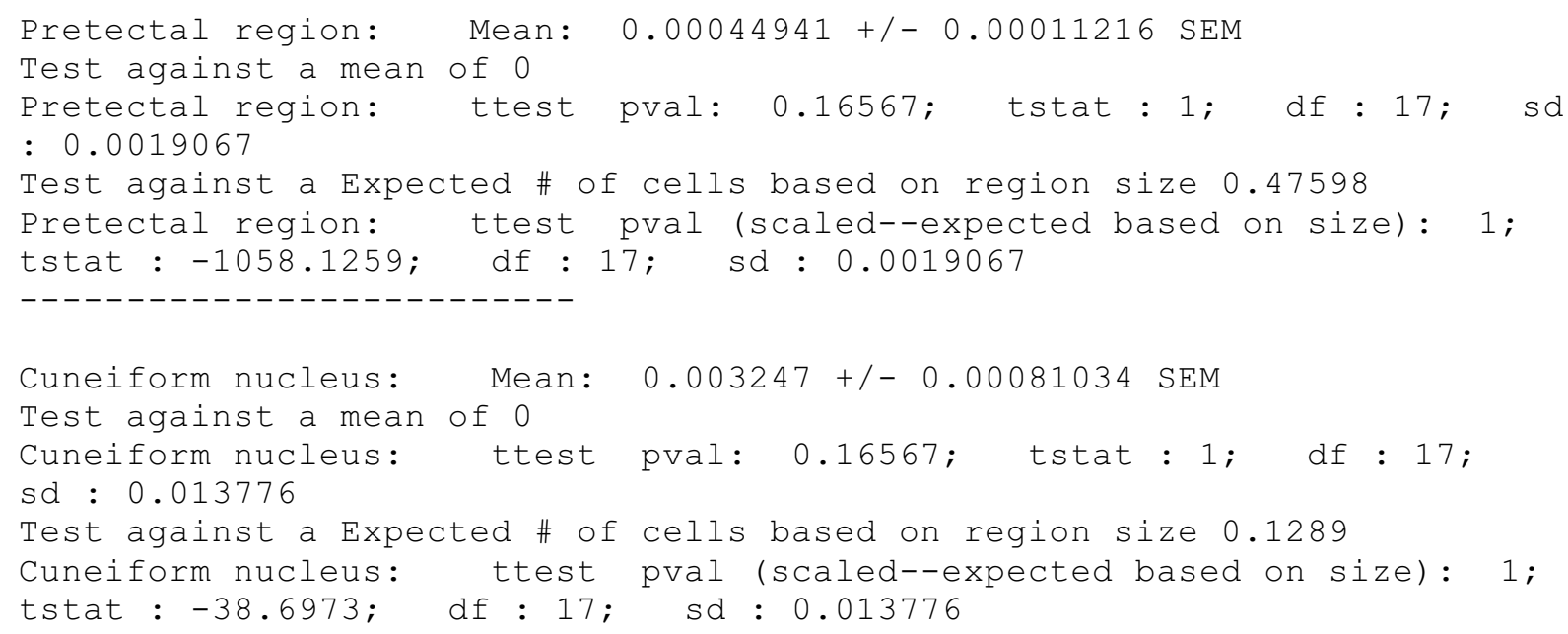

This is a confidential document and must not be discussed with others, forwarded in any form, or posted on websites without the express written consent of eNeuro. 
Test against a Expected \# of cells based on region size 0.0056501 Edinger-Westphal nucleus: size): 1 ; tstat : - Inf; ttest pval (scaled--expected based on df : $17 ;$ sd : 0

Trochlear nucleus: Mean: $0+/-0$ SEM

Test against a mean of 0

Trochlear nucleus: ttest pval: NaN; tstat : NaN; df : 17; sd : 0

Test against a Expected \# of cells based on region size 0.0015415

Trochlear nucleus: ttest pval (scaled--expected based on size): 1; tstat : -Inf; df : 17; sd : 0

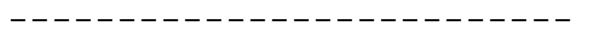

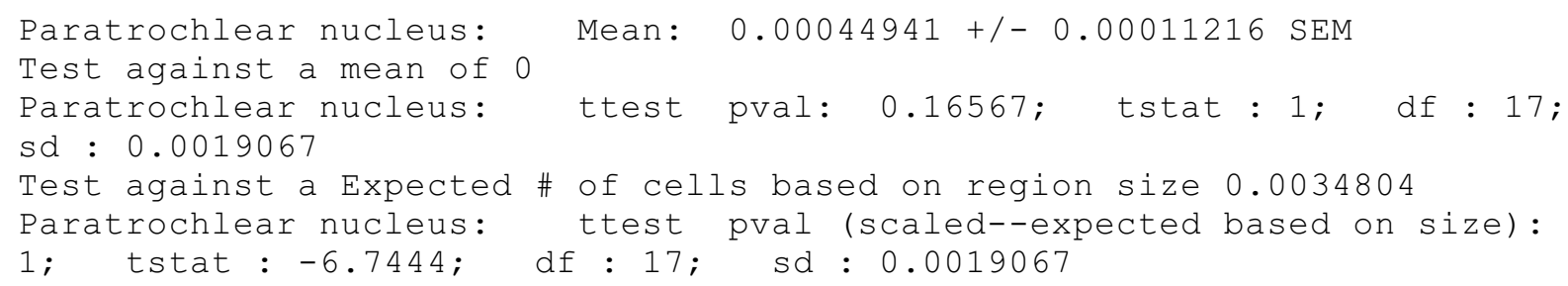

Lateral terminal nucleus of the accessory optic tract: Mean: $0.0042103+/-0.0010508$ SEM

Test against a mean of 0

Lateral terminal nucleus of the accessory optic tract: ttest pval: 0.16567 ; tstat : 1 ; df : 17; sd : 0.017863

Test against a Expected \# of cells based on region size 0.004044 Lateral terminal nucleus of the accessory optic tract: ttest pval (scaled--expected based on size): 0.48447 ; tstat : 0.039508 ; df : $17 ;$ sd : 0.017863

This is a confidential document and must not be discussed with others, forwarded in any form, or posted on websites without the express written consent of eNeuro. 
Dorsal terminal nucleus of the accessory optic tract: Mean: 0 +/- 0 SEM

Test against a mean of 0

Dorsal terminal nucleus of the accessory optic tract: ttest pval:

$\mathrm{NaN}$; tstat : NaN; df : 17; sd : 0

Test against a Expected \# of cells based on region size 0.0029235

Dorsal terminal nucleus of the accessory optic tract: ttest pval

(scaled--expected based on size): 1; tstat : -Inf; df : 17; sd : 0

Medial terminal nucleus of the accessory optic tract: Mean: 0 +/- 0 SEM

Test against a mean of 0

Medial terminal nucleus of the accessory optic tract: ttest pval:

NaN; tstat : NaN; df : 17; sd : 0

Test against a Expected \# of cells based on region size 0.0094734

Medial terminal nucleus of the accessory optic tract: ttest pval

(scaled--expected based on size): 1; tstat : -Inf; df : 17; sd : 0

Superior colliculus, sensory related: Mean: 0 +/- 0 SEM

Test against a mean of 0

Superior colliculus, sensory related: ttest pval: NaN; tstat :

NaN; df : 17; sd : 0

Test against a Expected \# of cells based on region size 0.47634

Superior colliculus, sensory related: ttest pval (scaled--expected

based on size): 1; tstat : -Inf; df : 17; sd : 0

Inferior colliculus: Mean: $0.020925+/-0.0052222$ SEM

Test against a mean of 0

Inferior colliculus: ttest pval: 0.16567 ; tstat : 1 ; df : 17;

sd : 0.088777

Test against a Expected \# of cells based on region size 0.96665

Inferior colliculus: ttest pval (scaled--expected based on size): 1 ; tstat : $-45.1962 ;$ df : 17; sd : 0.088777

Nucleus of the brachium of the inferior colliculus: Mean: 0.010462 $+/-0.0026111$ SEM

Test against a mean of 0

Nucleus of the brachium of the inferior colliculus: ttest pval:

0.16567 ; tstat : 1; df : 17; sd : 0.044388

Test against a Expected \# of cells based on region size 0.029602

Nucleus of the brachium of the inferior colliculus: ttest pval

(scaled--expected based on size): 0.95753; tstat : -1.8293 ; df :

17; sd : 0.044388

Nucleus sagulum: Mean: 0 +/- 0 SEM

This is a confidential document and must not be discussed with others, forwarded in any form, or posted on websites without the express written consent of eNeuro. 
$\begin{array}{lll}\text { Test against a mean of } 0 & & \\ \text { Nucleus sagulum: } & \text { test pval: NaN; tstat : NaN; df : } 17 ; \text { sd : }\end{array}$ 0

Test against a Expected \# of cells based on region size 0.021344

Nucleus sagulum: ttest pval (scaled--expected based on size): 1;

tstat : -Inf; df : $17 ;$ sd : 0

-------------------------

Parabigeminal nucleus: Mean: $0+/-0$ SEM

Test against a mean of 0

Parabigeminal nucleus: ttest pval: NaN; tstat : NaN; df: 17;

sd : 0

Test against a Expected \# of cells based on region size 0.0096839

Parabigeminal nucleus: ttest pval (scaled--expected based on size):

$1 ;$ tstat : -Inf; df : $17 ;$ sd : 0

Midbrain trigeminal nucleus: Mean: 0 +/- 0 SEM

Test against a mean of 0

Midbrain trigeminal nucleus: ttest pval: NaN; tstat : NaN; df :

$17 ;$ sd : 0

Test against a Expected \# of cells based on region size 0.0022003

Midbrain trigeminal nucleus: ttest pval (scaled--expected based on

size): 1; tstat : -Inf; df : 17; sd : 0

Subcommissural organ: Mean: $0+/-0$ SEM

Test against a mean of 0

Subcommissural organ: ttest pval: NaN; tstat : NaN; df: 17;

sd : 0

Test against a Expected \# of cells based on region size 0.0092832

Subcommissural organ: ttest pval (scaled--expected based on size) :

$1 ;$ tstat : -Inf; df : 17; sd : 0

------------------------

Substantia nigra, compact part: Mean: 0.00044941 +/-0.00011216 SEM

Test against a mean of 0

Substantia nigra, compact part: ttest pval: 0.16567 ; tstat : 1;

df : 17; sd : 0.0019067

Test against a Expected \# of cells based on region size 0.043051

Substantia nigra, compact part: ttest pval (scaled--expected based on size): 1; tstat : $-94.7958 ;$ df : 17; sd : 0.0019067

Pedunculopontine nucleus: $\quad$ Mean: $0.0012484+/-0.00031157$ SEM

Test against a mean of 0

Pedunculopontine nucleus: ttest pval: 0.16567; tstat : 1; df :

17 isd : 0.0052967

Test against a Expected \# of cells based on region size 0.2086

Pedunculopontine nucleus: ttest pval (scaled--expected based on

size): 1; tstat : $-166.0893 ;$ df : 17; sd : 0.0052967

This is a confidential document and must not be discussed with others, forwarded in any form, or posted on websites without the express written consent of eNeuro. 
Interfascicular nucleus raphe: Mean: $0+/-0$ SEM

Test against a mean of 0

Interfascicular nucleus raphe: ttest pval: NaN; tstat : NaN; df : 17 ; sd : 0

Test against a Expected \# of cells based on region size 0.020156

Interfascicular nucleus raphe: ttest pval (scaled--expected based on size): 1; tstat : -Inf; df : 17; sd : 0

Interpeduncular nucleus: Mean: 0.00089881 +/- 0.00022431 SEM Test against a mean of 0 Interpeduncular nucleus: 17 ; sd : 0.0038133 Test against a Expected \# of cells based on region size 0.079699 Interpeduncular nucleus: ttest pval (scaled--expected based on size): 1; tstat : $-87.671 ;$ df : 17; sd : 0.0038133

Rostral linear nucleus raphe: Mean: $0+/-0$ SEM

Test against a mean of 0

Rostral linear nucleus raphe: ttest pval: NaN; tstat : NaN; df : 17 i sd : 0

Test against a Expected \# of cells based on region size 0.016071

Rostral linear nucleus raphe: ttest pval (scaled--expected based on size): 1; tstat : -Inf; df : 17; sd : 0

$------------------------$

Central linear nucleus raphe: Mean: $0+/-0$ SEM

Test against a mean of 0

Central linear nucleus raphe: ttest pval: NaN; tstat : NaN; df : 17 ; sd : 0

Test against a Expected \# of cells based on region size 0.020332

Central linear nucleus raphe: ttest pval (scaled--expected based on size): 1; tstat : -Inf; df : 17; sd : 0

Dorsal nucleus raphe: Mean: $0.0036964+/-0.0008115$ SEM

Test against a mean of 0

Dorsal nucleus raphe: ttest pval: 0.1357; tstat : 1.1368; df:

17 ; sd : 0.013795

Test against a Expected \# of cells based on region size 0.033422

Dorsal nucleus raphe: ttest pval (scaled--expected based on size): $1 ;$ tstat : $-9.1417 ;$ df $: 17 ;$ sd : 0.013795

$-------------------------$

Primary motor area: Mean: $0.006686+/-0.0012921$ SEM

Test against a mean of 0

Primary motor area: ttest pval: 0.10693 ; tstat : 1.2913; df :

17 isd : 0.021966

Test against a Expected \# of cells based on region size 2.6186

Primary motor area: ttest pval (scaled--expected based on size): 1;

This is a confidential document and must not be discussed with others, forwarded in any form, or posted on websites without the express written consent of eNeuro. 
tstat : $-504.4714 ;$ df $: 17 ;$ sd : 0.021966

Secondary motor area: Mean: $0.074756+/-0.016114$ SEM

Test against a mean of 0

Secondary motor area: ttest pval: 0.13147; tstat : 1.1578; df : 17; sd : 0.27393

Test against a Expected \# of cells based on region size 2.9481

Secondary motor area: ttest pval (scaled--expected based on size):

$1 ;$ tstat : $-44.5026 ;$ df : 17; sd : 0.27393

\author{
primary somatosensory area: $\quad$ Mean: 0.0027917 +/- 0.0004482 SEM \\ Test against a mean of 0 \\ primary somatosensory area: ttest pval: 0.069247; tstat : 1.5545; \\ df : 17; sd : 0.0076194 \\ Test against a Expected \# of cells based on region size 5.5681 \\ primary somatosensory area: ttest pval (scaled--expected based on \\ size): 1; tstat : -3098.8479 ; df : 17; sd : 0.0076194
}

\begin{abstract}
Supplemental somatosensory area: Mean: $0.0063329+/-0.0011044$ SEM
Test against a mean of 0

Supplemental somatosensory area: ttest pval: 0.085266 ; tstat :

1.4311; df : 17; sd : 0.018775

Test against a Expected \# of cells based on region size 2.0429

Supplemental somatosensory area: ttest pval (scaled--expected based on size): 1; tstat : $-460.2201 ;$ df : 17; sd : 0.018775
\end{abstract}

Gustatory areas: Mean: 0.0050837 +/-0.00097392 SEM

Test against a mean of 0

Gustatory areas: ttest pval: 0.10502; tstat : 1.3027; df : 17;

sd : 0.016557

Test against a Expected \# of cells based on region size 0.38663

Gustatory areas: ttest pval (scaled--expected based on size): 1;

tstat : $-97.7722 ;$ df : 17; sd : 0.016557

Visceral area: Mean: $0.013856+/-0.0013483$ SEM

Test against a mean of 0

Visceral area: ttest pval: 0.010045; tstat : 2.5647; df: 17;

sd : 0.022921

Test against a Expected \# of cells based on region size 0.52504

Visceral area: ttest pval (scaled--expected based on size): 1;

tstat : $-94.6193 ;$ df : 17; sd : 0.022921

Auditory areas: Mean: 0.0047348 +/- 0.00071797 SEM

Test against a mean of 0

Auditory areas: ttest pval: 0.05908 ; tstat : 1.6458; df: 17;

sd : 0.012206

This is a confidential document and must not be discussed with others, forwarded in any form, or posted on websites without the express written consent of eNeuro. 
Test against a Expected \# of cells based on region size 1.3473

Auditory areas: ttest pval (scaled--expected based on size): 1; tstat : $-466.6904 ;$ df : 17; sd : 0.012206

Visual areas: Mean: $0.008255+/-0.0014136$ SEM

Test against a mean of 0

Visual areas: ttest pval: 0.081613; tstat : 1.4574; df : 17;

sd : 0.024031

Test against a Expected \# of cells based on region size 3.1429

Visual areas: ttest pval (scaled--expected based on size): 1;

tstat : $-553.4224 ;$ df : 17; sd : 0.024031

Anterior cingulate area:

Mean: $0.010989+/-0.0024326$ SEM

Test against a mean of 0

Anterior cingulate area:

df : 17; sd : 0.041355

test pval: 0.13762 ; tstat : 1.1274;

Test against a Expected \# of cells based on region size 1.3819

Anterior cingulate area: ttest pval (scaled--expected based on size): 1; tstat : $-140.646 ;$ df : 17; sd : 0.041355

Prelimbic area: Mean: $0.0012484+/-0.00031157$ SEM

Test against a mean of 0

Prelimbic area: ttest pval: 0.16567 ; tstat : 1; df : 17; sd: 0.0052967

Test against a Expected \# of cells based on region size 0.54511

Prelimbic area: ttest pval (scaled--expected based on size): 1;

tstat : $-435.6325 ;$ df : 17; sd : 0.0052967

Infralimbic area: $\quad$ Mean: 0.014772 +/- 0.0015668 SEM

Test against a mean of 0

Infralimbic area: ttest pval: 0.01546; tstat : 2.3531; df: 17;

sd : 0.026635

Test against a Expected \# of cells based on region size 0.20061

Infralimbic area: ttest pval (scaled--expected based on size): 1;

tstat : $-29.6022 ;$ df : 17; sd : 0.026635

Orbital area: Mean: $0.030667+/-0.0049647$ SEM

Test against a mean of 0

Orbital area: ttest pval: 0.070793; tstat : 1.5416; df : 17;

sd : 0.0844

Test against a Expected \# of cells based on region size 1.3105

Orbital area: ttest pval (scaled--expected based on size): 1;

tstat : $-64.3337 ;$ df : 17; sd : 0.0844

Agranular insular area: Mean: 0.074015 +/-0.0062399 SEM

Test against a mean of 0

This is a confidential document and must not be discussed with others, forwarded in any form, or posted on websites without the express written consent of eNeuro. 


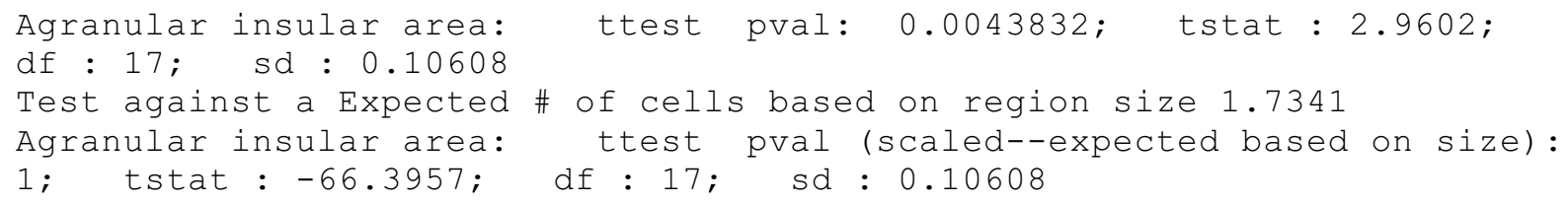

This is a confidential document and must not be discussed with others, forwarded in any form, or posted on websites without the express written consent of eNeuro. 


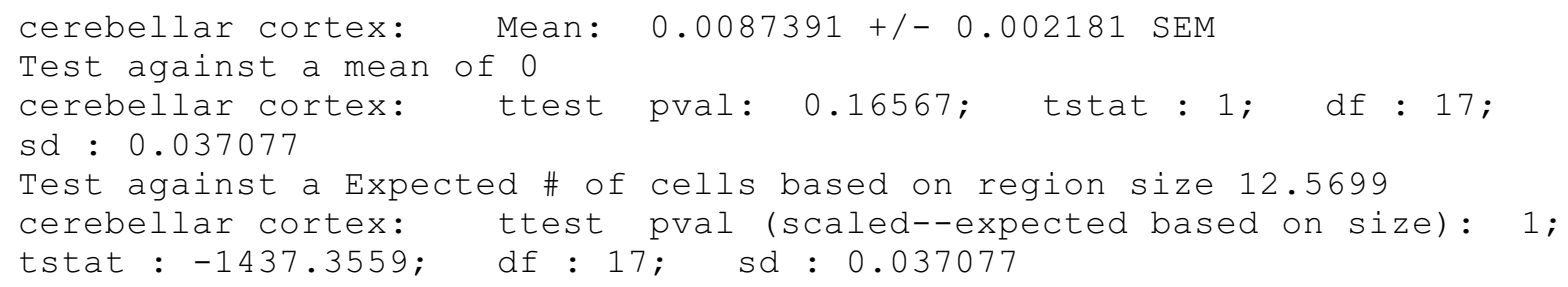

This is a confidential document and must not be discussed with others, forwarded in any form, or posted on websites without the express written consent of eNeuro. 
Subparafascicular nucleus: Test against a mean of 0

Subparafascicular nucleus: 17; sd : 0

Test against a Expected \# of Subparafascicular nucleus: size): 1; tstat : -Inf;

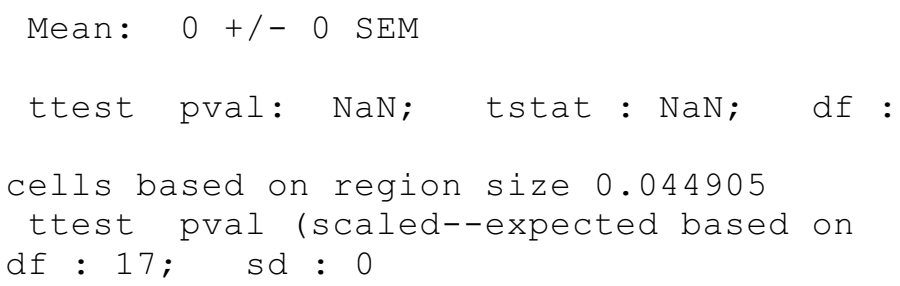

This is a confidential document and must not be discussed with others, forwarded in any form, or posted on websites without the express written consent of eNeuro. 
Test against a Expected \# of cells based on region size 0.57039

Anterior group of the dorsal thalamus: ttest pval (scaled--expected based on size): 1 ; tstat : -Inf; df : 17; sd : 0

Medial group of the dorsal thalamus: Mean: $0.00711+/-0.00096338$

SEM

Test against a mean of 0

Medial group of the dorsal thalamus: ttest pval: 0.041505; tstat : 1.8419; df : 17; sd : 0.016378

Test against a Expected \# of cells based on region size 0.45517

Medial group of the dorsal thalamus: ttest pval (scaled--expected

based on size): 1; tstat : -116.072; df : 17; sd : 0.016378

Midline group of the dorsal thalamus: Mean: $0.11222+/-0.010624$ SEM

Test against a mean of 0

Midline group of the dorsal thalamus: ttest pval: 0.0086697;

tstat : 2.6359; df : 17; sd : 0.18062

Test against a Expected \# of cells based on region size 0.28566

Midline group of the dorsal thalamus: ttest pval (scaled--expected

based on size): 0.99961; tstat : -4.0743 ; df : 17; sd : 0.18062

Intralaminar nuclei of the dorsal thalamus: Mean: $0.074545+/-$

0.012797 SEM

Test against a mean of 0

Intralaminar nuclei of the dorsal thalamus: ttest pval: 0.082118;

tstat : 1.4537; df : 17; sd : 0.21756

Test against a Expected \# of cells based on region size 0.36311

Intralaminar nuclei of the dorsal thalamus: ttest pval (scaled-expected based on size): 0.99998; tstat : -5.6275 ; df : 17; sd : 0.21756

Reticular nucleus of the thalamus: Mean: 0.87295 +/- 0.14077 SEM

Test against a mean of 0

Reticular nucleus of the thalamus: ttest pval: 0.070059; tstat :

1.5477; df : 17; sd : 2.3931

Test against a Expected \# of cells based on region size 0.49022

Reticular nucleus of the thalamus: ttest pval (scaled--expected

based on size): 0.25328 ; tstat : 0.67855; df : 17; sd : 2.3931

Geniculate group, ventral thalamus: Mean: 0.076796 +/- 0.0089385 SEM Test against a mean of 0

Geniculate group, ventral thalamus: ttest pval: 0.023384; tstat : 2.1442; df : 17; sd : 0.15195

Test against a Expected \# of cells based on region size 0.17279

Geniculate group, ventral thalamus: ttest pval (scaled--expected

based on size): 0.99209; tstat: -2.6803; df : 17; sd : 0.15195

This is a confidential document and must not be discussed with others, forwarded in any form, or posted on websites without the express written consent of eNeuro. 
Epithalamus: Mean: $0.00089881+/-0.00022431$ SEM

Test against a mean of 0

Epithalamus: ttest pval: 0.16567; tstat : 1; df : 17; sd :

0.0038133

Test against a Expected \# of cells based on region size 0.22435

Epithalamus: ttest pval (scaled--expected based on size): 1;

tstat : $-248.6063 ;$ df : 17; sd : 0.0038133

Caudoputanum: Mean: $6.5636+/-0.63899$ SEM

Test against a mean of 0

Caudoputanum: ttest pval: 0.010071; tstat : 2.5635; df : 17;

sd : 10.8628

Test against a Expected \# of cells based on region size 6.55

Caudoputanum: ttest pval (scaled--expected based on size): 0.49791;

tstat : $0.0053252 ;$ df : 17; sd : 10.8628

Nucleus accumbens: Mean: $0.14857+/-0.014201$ SEM

Test against a mean of 0

Nucleus accumbens: ttest pval: 0.0091294; tstat : 2.611; df :

17; sd : 0.24141

Test against a Expected \# of cells based on region size 0.99813

Nucleus accumbens: ttest pval (scaled--expected based on size): 1;

tstat : $-14.9302 ;$ df : 17; sd : 0.24141

Fundus of striatum: Mean: $0.11367+/-0.011312$ SEM

Test against a mean of 0

Fundus of striatum: ttest pval: 0.011294 ; tstat : 2.5077; df :

17; sd : 0.19231

Test against a Expected \# of cells based on region size 0.093834

Fundus of striatum: ttest pval (scaled--expected based on size):

$0.33359 ;$ tstat : 0.43761; df : 17; sd : 0.19231

Olfactory Tubercle: Mean: $0.22479+/-0.044624$ SEM

Test against a mean of 0

Olfactory Tubercle: ttest pval: 0.11284; tstat : 1.2572; df :

17; sd : 0.75861

Test against a Expected \# of cells based on region size 0.84157

Olfactory Tubercle: ttest pval (scaled--expected based on size):

0.99847; tstat : -3.4494 ; df : 17; sd : 0.75861

Lateral septal nucleus: Mean: $0.14769+/-0.012135$ SEM

Test against a mean of 0

Lateral septal nucleus: ttest pval: 0.0037178; tstat : 3.0375;

df : 17; sd : 0.20629

Test against a Expected \# of cells based on region size 0.79092

Lateral septal nucleus: ttest pval (scaled--expected based on size):

This is a confidential document and must not be discussed with others, forwarded in any form, or posted on websites without the express written consent of eNeuro. 
$1 ;$ tstat : $-13.2287 ;$ df $: 17 ;$ sd $: 0.20629$

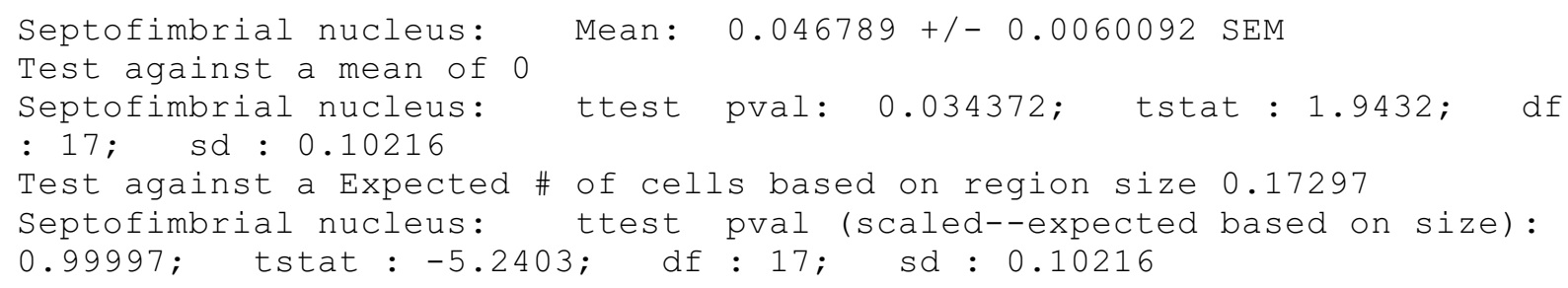

------------------------

Septohippocampal nucleus: Mean: $0+/-0$ SEM

Test against a mean of 0

Septohippocampal nucleus: ttest pval: NaN; tstat : NaN; df :

17; sd : 0

Test against a Expected \# of cells based on region size 0.0097178

Septohippocampal nucleus:

size): 1; tstat : -Inf;

ttest pval (scaled--expected based on

df : 17; sd : 0

Anterior amygdalar area: Mean: 0.25848 +/- 0.021208 SEM

Test against a mean of 0

Anterior amygdalar area:

df : 17; sd : 0.36054

Test against a Expected \# of cells based on region size 0.10747

Anterior amygdalar area: ttest pval (scaled--expected based on

size): 0.046736 ; tstat : $1.7769 ;$ df : 17; sd : 0.36054

Bed nucleus of the accessory olfactory tract: Mean: 0.0012484 +/0.00031157 SEM

Test against a mean of 0

Bed nucleus of the accessory olfactory tract: test pval: 0.16567;

tstat : 1; df : 17; sd : 0.0052967

Test against a Expected \# of cells based on region size 0.0054463

Bed nucleus of the accessory olfactory tract: ttest pval (scaled-expected based on size): 0.99815 ; tstat : -3.3625 ; df : 17; sd: 0.0052967

Central amygdalar nucleus: Mean: $11.3864+/-0.59917$ SEM

Test against a mean of 0

Central amygdalar nucleus: ttest pval: 9.4177e-05; tstat :

4.7427; df : 17; sd : 10.1858

Test against a Expected \# of cells based on region size 0.2958

Central amygdalar nucleus: test pval (scaled--expected based on size): 0.00012234 ; tstat : 4.6195; df : 17; sd : 10.1858

Intercalated amygdalar nucleus: Mean: $0.43087+/-0.039931$ SEM Test against a mean of 0

This is a confidential document and must not be discussed with others, forwarded in any form, or posted on websites without the express written consent of eNeuro. 
Intercalated amygdalar nucleus: ttest pval: 0.0077006; tstat : 2.6929; df : 17; sd : 0.67883

Test against a Expected \# of cells based on region size 0.039897

Intercalated amygdalar nucleus: ttest pval (scaled--expected based on size): 0.012874 ; tstat : 2.4436; df : 17; sd : 0.67883

Medial amygdalar nucleus: Mean: 20.0323 +/- 1.3142 SEM

Test against a mean of 0

Medial amygdalar nucleus: ttest pval: 0.00070912; tstat : 3.8041;

df : 17; sd : 22.3418

Test against a Expected \# of cells based on region size 0.5476

Medial amygdalar nucleus: ttest pval (scaled--expected based on

size): 0.00088875 ; tstat : 3.7001; df : 17; sd : 22.3418

Globus pallidus, external segment: Mean: $3.2419+/-0.30863$ SEM

Test against a mean of 0

Globus pallidus, external segment: ttest pval: 0.0089331; tstat :

2.6215; df : 17; sd : 5.2467

Test against a Expected \# of cells based on region size 0.40648

Globus pallidus, external segment: ttest pval (scaled--expected

based on size): 0.017442; tstat : 2.2928; df : 17; sd : 5.2467

Globus pallidus, internal segment: Mean: $1.4163+/-0.11891$ SEM

Test against a mean of 0

Globus pallidus, internal segment: ttest pval: 0.004272; tstat :

2.9723; df : 17; sd : 2.0215

Test against a Expected \# of cells based on region size 0.1381

Globus pallidus, internal segment: ttest pval (scaled--expected

based on size): 0.00787 ; tstat : 2.6825; df : 17; sd : 2.0215

Substantia innominata: Mean: 2.5134 +/- 0.19436 SEM

Test against a mean of 0

Substantia innominata: ttest pval: 0.0024738 ; tstat : 3.2273;

df : 17; sd : 3.3041

Test against a Expected \# of cells based on region size 0.65833

Substantia innominata: ttest pval (scaled--expected based on size):

0.014584 ; tstat : 2.382; df : 17; sd : 3.3041

Magnocellular nucleus: Mean: $0.27731+/-0.021054$ SEM

Test against a mean of 0

Magnocellular nucleus:

df : 17; sd : 0.35792

Test against a Expected \# of cells based on region size 0.079227

Magnocellular nucleus: ttest pval (scaled--expected based on size): $0.015619 ;$ tstat : 2.348; df : 17; sd : 0.35792

This is a confidential document and must not be discussed with others, forwarded in any form, or posted on websites without the express written consent of eNeuro. 
Medial septal nucleus: Test against a mean of 0 Medial septal nucleus: : 17; sd : 0.32167 Test against a Expected \# Medial septal nucleus: 0.10943 ; tstat : 1.2767 ;

Diagonal band nucleus: Test against a mean of 0 Diagonal band nucleus: df : 17; sd : 0.57353 Test against a Expected \# Diagonal band nucleus: 0.013211; tstat : 2.4308;

Mean: $0.48947+/-0.033737$ SEM

test pval: 0.0010556 ; tstat : 3.6208;

of cells based on region size 0.16087

ttest pval (scaled--expected based on size): $0.013211 ;$ tstat : $2.4308 ;$

Triangular nucleus of septum: Mean: $0.0064051+/-0.00088501$ SEM Test against a mean of 0 Triangular nucleus of septum: ttest pval: 0.044312 ; tstat : 1.8062; df : 17; sd : 0.015045 Test against a Expected \# of cells based on region size 0.11501 Triangular nucleus of septum: ttest pval (scaled--expected based on size): 1; tstat : $-30.6272 ;$ df : 17; sd : 0.015045

BNST: Mean: $0.61112+/-0.043287$ SEM

Test against a mean of 0

BNST: ttest pval: 0.0013042 ; tstat : 3.5234 ; df : 17; sd : 0.73588

Test against a Expected \# of cells based on region size 0.33723

BNST: ttest pval (scaled--expected based on size): 0.066372;

tstat : 1.5791; df : 17; sd : 0.73588

MainolfactoryBulb: Mean: $0.25171+/-0.038879$ SEM

Test against a mean of 0

MainolfactoryBulb: ttest pval: 0.062276 ; tstat : 1.6158 ; df :

17 ; sd : 0.66094

Test against a Expected \# of cells based on region size 4.2909

MainolfactoryBulb: ttest pval (scaled--expected based on size): 1; tstat : $-25.9276 ;$ df $: 17 ;$ sd : 0.66094

AccessoryolfactoryBulb: Mean: 0.92978 +/-0.097154 SEM

Test against a mean of 0

AccessoryolfactoryBulb: ttest pval: 0.014397 ; tstat : 2.3884;

df : 17; sd : 1.6516

Test against a Expected \# of cells based on region size 0.16047

AccessoryolfactoryBulb: ttest pval (scaled--expected based on size): $0.032296 ;$ tstat : $1.9762 ;$ df : 17; sd : 1.6516

This is a confidential document and must not be discussed with others, forwarded in any form, or posted on websites without the express written consent of eNeuro. 
AnteriorolfactoryNucleus:

Test against a mean of 0

AnteriorolfactoryNucleus:

df : 17; sd : 0.011804

Test against a Expected \# of

AnteriorolfactoryNucleus:

size): 1 ; tstat : -418.465 ;
Mean: $0.0037733+/-0.00069434$ SEM

test pval: 0.096383; tstat : 1.3562;

cells based on region size 1.168

ttest pval (scaled--expected based on df : 17; sd : 0.011804

Taenia tecta: Mean: 0.003908 +/- 0.00062019 SEM

Test against a mean of 0

Taenia tecta: test pval: 0.06712 ; tstat : 1.5726; df: 17;

sd : 0.010543

Test against a Expected \# of cells based on region size 0.31398

Taenia tecta: ttest pval (scaled--expected based on size): 1;

tstat : $-124.7746 ;$ df : 17; sd : 0.010543

Dorsal peduncular area: Mean: $0.001602+/-0.0002845$ SEM

Test against a mean of 0

Dorsal peduncular area: ttest pval: 0.088968; tstat : 1.4053;

df : 17; sd : 0.0048365

Test against a Expected \# of cells based on region size 0.12319

Dorsal peduncular area: ttest pval (scaled--expected based on size): 1 i tstat : -106.657 ; df : 17; sd : 0.0048365

------------------------

Piriform area: Mean: $1.0382+/-0.05887$ SEM

Test against a mean of 0

Piriform area: ttest pval: $0.00019501 ;$ tstat : 4.4014; df: 17;

sd : 1.0008

Test against a Expected \# of cells based on region size 2.6015

Piriform area: ttest pval (scaled--expected based on size): 1;

tstat : $-6.6274 ;$ df $: 17 ;$ sd : 1.0008

Nucleus of the lateral olfactory tract: Mean: $0.21477+/-0.033776$

SEM

Test against a mean of 0

Nucleus of the lateral olfactory tract: ttest pval: 0.06548;

tstat : 1.5869; df: $17 ;$ sd : 0.5742

Test against a Expected \# of cells based on region size 0.070684

Nucleus of the lateral olfactory tract: ttest pval (scaled--expected

based on size): 0.15097 ; tstat : 1.0646; df : 17; sd : 0.5742

Cortical amygdalar area: Mean: $3.9576+/-0.26051$ SEM

Test against a mean of 0

Cortical amygdalar area:

df : $17 ;$ sd : 4.4286

test pval: 0.0007289 ; tstat : 3.7914;

This is a confidential document and must not be discussed with others, forwarded in any form, or posted on websites without the express written consent of eNeuro. 
Test against a Expected \# of cells based on region size 0.70976

Cortical amygdalar area: ttest pval (scaled--expected based on

size): 0.0031734 ; tstat : 3.1114; df : 17; sd : 4.4286

Piriform-amygdalar area: Mean: 0.37813 +/-0.021107 SEM

Test against a mean of 0

Piriform-amygdalar area:

df : 17; sd : 0.35881

Test against a Expected \# of cells based on region size 0.25921

Piriform-amygdalar area: ttest pval (scaled--expected based on

size): $0.088843 ;$ tstat : $1.4062 ;$ df : 17; sd: 0.35881

-------------------------

Postpiriform transition area: Mean: $0.33813+/-0.030562$ SEM

Test against a mean of 0

Postpiriform transition area: ttest pval: 0.0066758 ; tstat :

2.7612; df : 17; sd : 0.51955

Test against a Expected \# of cells based on region size 0.30535

Postpiriform transition area: ttest pval (scaled--expected based on size): 0.3961 ; tstat : $0.26765 ;$ df : 17; sd : 0.51955

Claustrum: Mean: $0.0042561+/-0.00083431$ SEM

Test against a mean of 0

Claustrum: ttest pval: $0.11005 ;$ tstat : 1.2731; df: 17; sd: 0.014183

Test against a Expected \# of cells based on region size 0.12164

Claustrum: ttest pval (scaled--expected based on size): 1; tstat : $-35.113 ;$ df : 17; sd : 0.014183

Endopiriform nucleus-dorsal: Mean: $0.039201+/-0.0035084$ SEM

Test against a mean of 0

Endopiriform nucleus-dorsal: ttest pval: 0.0063029 ; tstat :

2.7885; df: 17; sd : 0.059643

Test against a Expected \# of cells based on region size 0.43775

Endopiriform nucleus-dorsal: ttest pval (scaled--expected based on size): 1; tstat : $-28.3509 ;$ df : 17; sd : 0.059643

Endopiriform nucleus-ventral: Mean: $0.085026+/-0.0061553$ SEM

Test against a mean of 0

Endopiriform nucleus-ventral: ttest pval: 0.0015377 ; tstat :

3.4474; df : 17; sd : 0.10464

Test against a Expected \# of cells based on region size 0.21637

Endopiriform nucleus-ventral: ttest pval (scaled--expected based on size): 0.99997 ; tstat : $-5.3255 ;$ df : 17; sd : 0.10464

Lateral amygdalar nucleus: Mean: 0.096562 +/-0.010483 SEM

Test against a mean of 0

This is a confidential document and must not be discussed with others, forwarded in any form, or posted on websites without the express written consent of eNeuro. 
Lateral amygdalar nucleus: ttest pval: 0.017234 ; tstat : 2.2988; df : 17; sd : 0.17821

Test against a Expected \# of cells based on region size 0.20089

Lateral amygdalar nucleus: ttest pval (scaled--expected based on size): 0.98814 ; tstat : -2.4838 ; df : 17; sd : 0.17821

Basolateral amygdalar nucleus-anterior: Mean: $0.59024+/-0.056393$ SEM

Test against a mean of 0

Basolateral amygdalar nucleus-anterior: ttest pval: 0.0091088; tstat : 2.6121; df : 17; sd : 0.95868

Test against a Expected \# of cells based on region size 0.17252

Basolateral amygdalar nucleus-anterior: ttest pval (scaled--expected based on size): 0.040991 ; tstat : 1.8486; df : 17; sd : 0.95868

Basolateral amygdalar nucleus-posterior: Mean: $0.26929+/-0.023909$

SEM

Test against a mean of 0

Basolateral amygdalar nucleus-posterior: ttest pval: 0.0060123; tstat : 2.811; df : 17; sd: 0.40645

Test against a Expected \# of cells based on region size 0.15594

Basolateral amygdalar nucleus-posterior: ttest pval (scaled-expected based on size): 0.1265; tstat : 1.1833; df : 17; sd : 0.40645

Basolateral amygdalar nucleus-ventral: Mean: 0.30243 +/- 0.0294 SEM Test against a mean of 0

Basolateral amygdalar nucleus-ventral: ttest pval: 0.0099945;

tstat : 2.5672; df : 17; sd : 0.4998

Test against a Expected \# of cells based on region size 0.091039

Basolateral amygdalar nucleus-ventral: ttest pval (scaled--expected based on size): 0.045276; tstat : 1.7944; df : 17; sd : 0.4998

Basomedial amygdalar nucleus -anterior: Mean: $1.5901+/-0.12207$ SEM Test against a mean of 0

Basomedial amygdalar nucleus -anterior: ttest pval: 0.0023508;

tstat : 3.251; df : 17; sd : 2.0751

Test against a Expected \# of cells based on region size 0.16715

Basomedial amygdalar nucleus -anterior: ttest pval (scaled--expected based on size): 0.0048844; tstat : 2.9093; df : 17; sd : 2.0751

Basomedial amygdalar nucleus-posterior: Mean: $1.623+/-0.12082$ SEM Test against a mean of 0

Basomedial amygdalar nucleus-posterior: ttest pval: 0.0018883;

tstat : 3.3525; df : 17; sd : 2.054

Test against a Expected \# of cells based on region size 0.15629

Basomedial amygdalar nucleus-posterior: ttest pval (scaled--expected

This is a confidential document and must not be discussed with others, forwarded in any form, or posted on websites without the express written consent of eNeuro. 


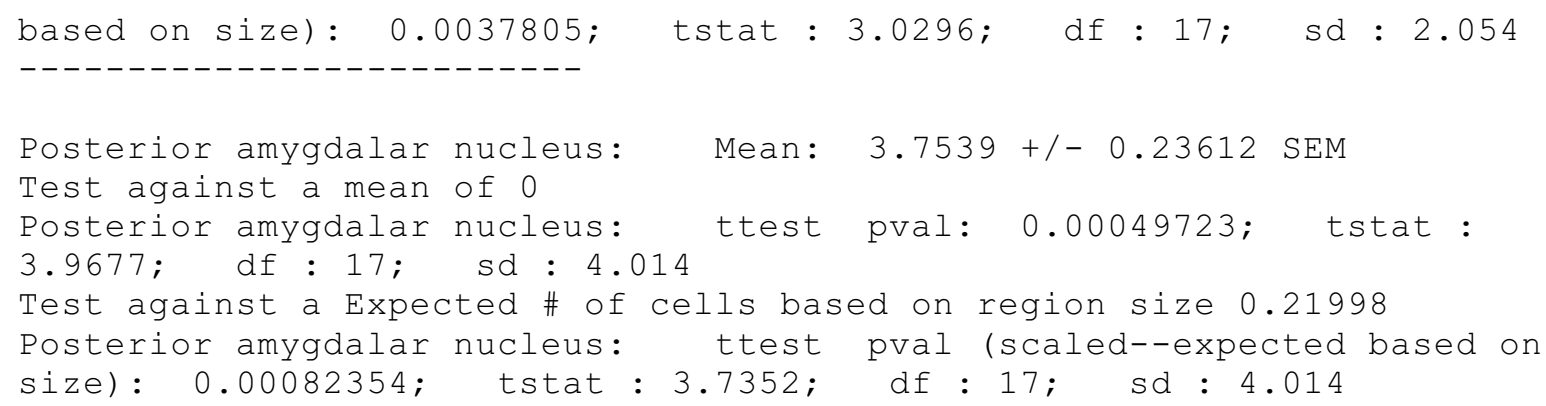

This is a confidential document and must not be discussed with others, forwarded in any form, or posted on websites without the express written consent of eNeuro. 
Region

Field CA1

FieldCa3

Dorsomedial nucleus of the hypothalamus

Lateral hypothalamic area

Lateral preoptic area

Tuberal nucleus

Medial mammillary nucleus

Ventral premammillary nucleus

Ventromedial hypothalamic nucleus

Reticular nucleus of the thalamus

Caudoputanum

Central amygdalar nucleus

Medial amygdalar nucleus

Globus pallidus, external segment

Globus pallidus, internal segment

Substantia innominata

Diagonal band nucleus

BNST

AccessoryOlfactoryBulb

Piriform area

Cortical amygdalar area

Piriform-amygdalar area

Postpiriform transition area

Basolateral amygdalar nucleus-anterior

Basolateral amygdalar nucleus-ventral

Basomedial amygdalar nucleus -anterior

Basomedial amygdalar nucleus-posterior

Posterior amygdalar nucleus

$\begin{array}{ccc}\text { Male average (\%) } & \text { Male SEM } & \text { Female average (\%) } \\ 3.75 & 1.26 & 0.68 \\ 6.79 & 3.24 & 2.53 \\ 0.59 & 0.36 & 0.32 \\ 2.15 & 0.83 & 2.01 \\ 0.55 & 0.14 & 0.19 \\ 0.02 & 0.01 & 1.98 \\ 0.23 & 0.10 & 0.57 \\ 0.03 & 0.01 & 1.08 \\ 0.52 & 0.21 & 2.15 \\ 0.27 & 0.15 & 1.66 \\ 5.78 & 3.21 & 8.27 \\ 14.61 & 3.79 & 9.04 \\ 12.95 & 3.51 & 29.19 \\ 2.49 & 1.08 & 4.49 \\ 1.14 & 0.53 & 1.90 \\ 3.61 & 1.37 & 1.58 \\ 0.74 & 0.22 & 0.24 \\ 0.86 & 0.28 & 0.40 \\ 1.68 & 0.70 & 0.19 \\ 1.70 & 0.32 & 0.29 \\ 4.13 & 1.42 & 2.90 \\ 0.60 & 0.12 & 0.16 \\ 0.58 & 0.22 & 0.06 \\ 1.13 & 0.38 & 0.05 \\ 0.54 & 0.21 & 0.08 \\ 1.98 & 0.82 & 1.27 \\ 1.52 & 0.43 & 1.83 \\ 3.80 & 1.33 & 2.56 \\ & & \end{array}$




$\begin{array}{cc}\text { Female SEM } & p \text {-value } \\ 0.32 & 0.0102 \\ 1.24 & 0.1272 \\ 0.15 & 0.3453 \\ 1.25 & 0.4462 \\ 0.08 & 0.0209 \\ 1.89 & 0.0625 \\ 0.45 & 0.3629 \\ 1.00 & 0.0375 \\ 1.41 & 0.1245 \\ 1.24 & 0.1364 \\ 4.62 & 0.2967 \\ 2.94 & 0.1303 \\ 10.68 & 0.0807 \\ 2.52 & 0.2509 \\ 0.89 & 0.2411 \\ 0.70 & 0.1146 \\ 0.14 & 0.0413 \\ 0.20 & 0.0962 \\ 0.12 & 0.0362 \\ 0.13 & 0.0002 \\ 1.52 & 0.2642 \\ 0.07 & 0.0038 \\ 0.04 & 0.0041 \\ 0.03 & 0.0007 \\ 0.04 & 0.01 \\ 0.63 & 0.251 \\ 1.01 & 0.3791 \\ 0.97 & 0.2437\end{array}$

